\title{
RESPIRATORY CONSEQUENCES OF PERIOPERATIVE COMPLICATIONS RELATED TO ANAESTHESIA
}

\author{
Gergely Fodor MD \\ PhD Thesis \\ Department of Medical Physics and Informatics \\ University of Szeged, Hungary \\ Doctoral School of Multidisciplinary Medical Sciences
}

Supervisor: Ferenc Peták

Szeged

2016 


\section{List of scientific papers included in this thesis}

I. Fodor G.H., Peták F., Érces D., Balogh Á. L., Babik B.: Lung mechanical changes following bronchoaspiration in a porcine model: differentiation of direct and indirect mechanisms. Respir Physiol Neurobiol. 2014;199:41-49.

II. Fodor G.H., Babik B., Czövek D., Doras C., Balogh Á.L., Bayat S., Habre W., Peták F.: Effects of fluid replacement on respiratory function: comparison of whole blood with colloid and crystalloid. Eur J Anaesthesiol. 2016; 33:34-41

\section{List of related but not included scientific papers}

III. Filep Á., Fodor G.H., Kun Szabó F., Tiszlavicz L., Rázga Z., Bozsó G. Bozóki Z.: Szabó G., Peták F.: Exposure to urban PM1 in rats: development of bronchial inflammation and airway hyperresponsiveness. Respir Res. 2016; 17:26

IV. Csorba Z., Peták F., Névery K., Tolnai J., Balogh Á.L., Rárosi F., Fodor G.H., Babik B.: Capnographic Parameters in Ventilated Patients: Correspondence with Airway and Lung Tissue Mechanics. Anaesth Analg. (Epub ahead print) 


\section{Table of contents}

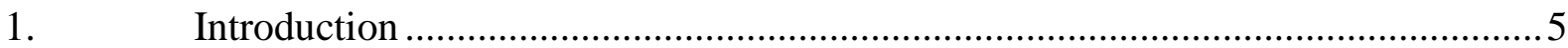

1.1. Perioperative respiratory complications ……………………………………......

1.2. Monitoring methods in anaesthesia: capnography and mechanical parameters ..........5

1.3. Aspiration as source of respiratory morbidity ...................................................

1.4. Cardiopulmonary effects of blood loss and fluid replacement ................................. 8

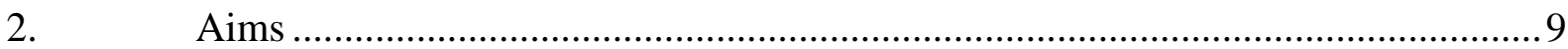

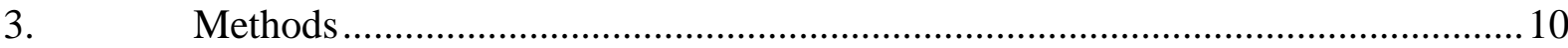

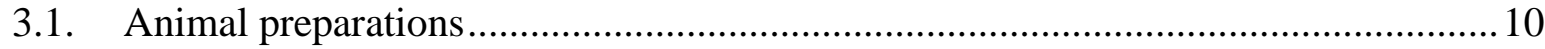

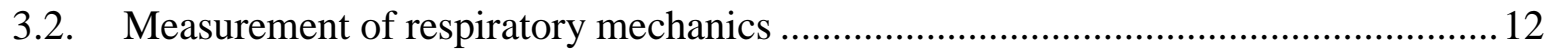

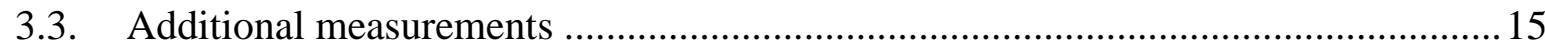

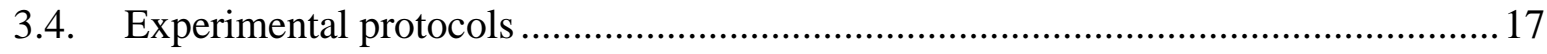

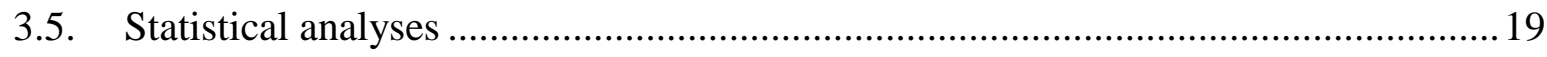

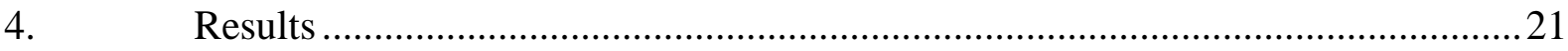

4.1. Pulmonary consequences of bronchoaspiration: results in pigs ............................ 21

4.2. Respiratory consequences of blood loss and fluid replacement: results in rats ........ 28

5

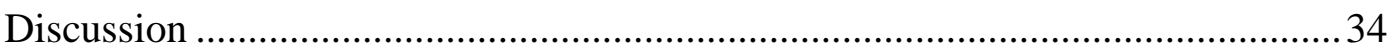

5.1. Effects of bronchoaspiration on respiratory function .......................................... 34

5.2. Respiratory effects of blood loss and fluid replacement........................................ 38

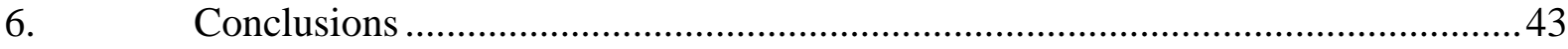

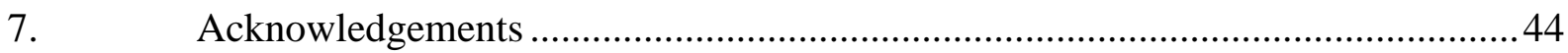

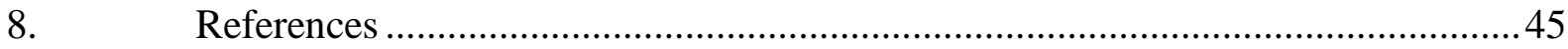




\section{Figures and tables}

Figure 1. Experimental set-up for lung measurement in pigs.......................................... 13

Figure 2. Experimental set-up for lung measurements in rats........................................ 14

Figure 3. Scheme of the experimental protocol in pigs................................................... 18

Figure 4. Scheme of the experimental protocol in rats..................................................... 19

Figure 5. Impedance spectra from a representative pig................................................. 21

Figure 6. Airway resistance (Raw), tissue damping $(\mathrm{G})$ and elastance $(\mathrm{H})$ for the affected and the intact lungs in Groups P10 and P4 under baseline conditions ( 0 min) and following

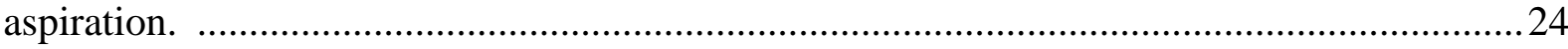

Figure 7. Relative changes in airway resistance (Raw), tissue damping $(\mathrm{G})$ and elastance $(\mathrm{H})$ in response to bronchoaspiration at 5, 60, 90 and $120 \mathrm{~min}$.

Figure 8. Association between the changes in airway resistance in the affected and the intact lung in the first 60 min following aspiration in Groups P4 and P10. 26

Figure 9. Third phase slope of the capnogram $\left(\mathrm{S}_{\text {III }}\right)$ and its normalized value $\left(\mathrm{S}_{\mathrm{nIII}}\right)$ for the affected and the intact lungs in Groups P10 and P4 under baseline conditions ( 0 min) and following aspiration

Figure 10. Changes in the airway and tissue mechanics relative to the baseline. 30

Figure 11. Systemic haemodynamic parameters. 31

Figure 12. Oedema indices following fluid replacement by various solutions. 32

Figure 13. Relationship between the changes in oedema index and in respiratory elastance in rats. 


\section{List of abbreviations in an alphabetic order}

ANOVA: analysis of variances

CI: cardiac index

$\mathrm{CO}_{2}$ : carbon-dioxide

ET: endotracheal

$\mathrm{FiO}_{2}$ : inspired oxygen fraction

G: tissue damping

$\mathrm{H}$ : tissue elastance

Hct: haematocrit

HES: hydroxyethyl-starch

HQ: Horowitz quotient

HR: heart rate

Iaw: airway inertance

$\mathrm{j}$ : imaginary unit

L: length of the wave-tube

MAP: mean arterial pressure

$\mathrm{P}_{1}$ : lateral pressure at the loudspeaker end of the wave-tube

$\mathrm{P}_{2}$ : lateral pressure at the tracheal end of the wave-tube

$\mathrm{PaCO}_{2}$ : partial pressure of carbon-dioxide
$\mathrm{PaO}_{2}$ : partial pressure of oxygen

PEEP: positive end-expiratory pressure

$\mathrm{P}_{\mathrm{L}, \mathrm{s}}$ : pressure in the left or right main bronchi with reference to the atmosphere

Raw: airway resistance

SEM: standard error of the mean

SIII: third phase slope of the capnogram

$\mathrm{S}_{\mathrm{nIII}}$ : normalized third phase slope of the capnogram

V: air flow

W/D: wet-to-dry lung weight ratio

$\mathrm{Z}_{0}$ : characteristic impedance of the wavetube

$\mathrm{Z}_{\mathrm{L}, \mathrm{s}}$ : input impedance of the right or left lung

$\mathrm{Z}_{\mathrm{rs}}$ : input impedance of the respiratory system

$\alpha:(2 / \pi) \arctan (\mathrm{H} / \mathrm{G})$

$\beta$ : empirical parameter for model fitting $\gamma$ : complex wave propagation number $\omega:$ angular frequency 


\section{Introduction}

\subsection{Perioperative respiratory complications}

General anaesthesia has been widely used since Morton's first public demonstration with ether as a general anaesthetic agent in 1846. It has been estimated that more than 230 million surgical procedures have been carried out under general anaesthesia worldwide every year [1]. Pulmonary complications are the second most frequent group of postoperative complications, contributing greatly to the postoperative morbidity, mortality and longer duration of hospitalization [2]. Previous studies have estimated their incidence in the range 2.0 to 7.9 per cent $[3,4]$. These adverse pulmonary consequences may manifest in various pathological conditions with respiratory failure, bronchospasm, aspiration pneumonitis and infections being the most important among them [4]. While some aspects of these conditions have been examined in great detail, the respiratory mechanical consequences have not been elucidated. The importance of such characterization stems from the fact that compromised respiratory mechanics ultimately deteriorates the gas exchange and therefore, worsens the life expectancy.

\subsection{Monitoring methods in anaesthesia: capnography and mechanical parameters}

Patient monitoring is an important tool for the anaesthetist in the early detection and prevention of these respiratory adverse events. The American Society for Anesthesiologists has provided guidelines for the minimal appropriate tools of patient monitoring [5]. These tools include monitoring of the vital signs of the respiratory and circulatory systems. The monitored vital signs for the respiratory system include capnography and measurement of the mechanical properties of the respiratory system.

\subsubsection{Capnographic measurements}

Capnography is a method for assessment of the expired carbon dioxide $\left(\mathrm{CO}_{2}\right)$ concentration. As it is a non-invasive method, it is a favourable way to assess changes in the gas-exchange properties of the respiratory system and lung emptying. The physiological capnogram has three phases. The first phase indicates the expiration from the anatomical dead space area, where no gas exchange takes place, and therefore has virtually no $\mathrm{CO}_{2}$ content, making this phase indistinguishable from the baseline of the $\mathrm{CO}_{2}$ trace. The second phase shows the 
exhalation of the $\mathrm{CO}_{2}$ front between the anatomical dead space and the alveolar compartment, resulting in a rapid elevation of the $\mathrm{CO}_{2}$ concentration. The third phase of the capnogram is due to the exhalation of the alveolar compartment. Physiologically, this phase is almost flat with a small upward slope $(\sim 1 \mathrm{mmHg} / \mathrm{s})$ ending in the end-tidal $\mathrm{CO}_{2}$, which is the highest point of the expiratory curve. The inspiration then causes a rapid drop of the capnogram back to the baseline.

The slope of the third phase $\left(\mathrm{S}_{\mathrm{III}}\right)$ is determined by the sequential emptying of the alveoli. Under physiological conditions, the alveoli are homogeneously ventilated periodically, while the circulation is continuous. This results in slightly lower $\mathrm{CO}_{2}$ concentrations from the alveoli emptying first and somewhat higher $\mathrm{CO}_{2}$ concentrations from the alveoli contributing to the later phase of expiration. This phenomenon is responsible for the slight slope. Under pathological conditions, the heterogeneity increases both in the $\mathrm{CO}_{2}$ content and in the emptying dynamics of the alveoli, resulting in greater within-breath changes of the exhaled $\mathrm{CO}_{2}$ concentration and subsequently a higher $\mathrm{S}_{\text {III. }}$.

\subsubsection{Respiratory mechanical measurements}

The mechanical properties of the respiratory system can be measured by using various methods and instrumentation. Most ventilators indicate an estimation of the respiratory resistance, calculated through the use of the interrupter technique. This technique utilizes the time domain analyses of the pressure changes at the airway opening following rapid occlusion of the inspiratory flow. A sudden drop in airway pressure reflects the airway properties, whereas a gradual decrease can be attributed to the tissue relaxation and pendelluft between the heterogeneous lung compartments. However, the interrupter airway resistance might not lead to correct estimations of the actual airway resistance due to the substantial involvement of components related to the resistance of the instrument itself and the respiratory tissues [6].

Another, more sophisticated method for measuring mechanical parameters is the forced oscillatory technique. This technique makes use of an external forcing pressure signal and through the measurement of pressure and flow signals, the input impedance of the respiratory system can be obtained. This impedance is a quantity which can be described with complex numbers, with the real part representing the resistive, while the imaginary part corresponds to

the elastic and inertive properties of the respiratory system. From this input impedance, 
various specific mechanical parameters can be obtained that are able to detect changes in various lung compartments with great sensitivity. While this technique provides detailed information about the airway and tissue mechanical properties, the on-line monitoring readily available with the interrupter technique is cumbersome due to the application of periodic external pressure excitations.

\subsection{Aspiration as source of respiratory morbidity}

Among the respiratory adverse effects during anaesthesia and intensive care, bronchoaspiration poses a major challenge for health professionals $[7,8]$. This syndrome has been reported to cause a high incidence of morbidity and mortality, involving up to $90 \%$ of the affected patients, depending on the extent of the involved lung regions [9-13]. Inhalation of the gastric contents into the lower respiratory tract induces a number of pulmonary syndromes, described originally by Mendelson et al., including acute aspiration pneumonitis caused by the acidity subsequent to chemical injury [14], and aspiration pneumonia resulting from the inhalation of pathogenic bacteria $[7,8,15]$.

The mechanisms responsible for the acute deterioration of lung function following aspiration of the gastric contents have not been fully clarified. Earlier results demonstrated the involvement of direct physiochemical processes leading to mucosal damage and desquamation [16, 17]. Another direct effect of gastric juice aspiration may be related to pepsin being cytotoxic to bronchial epithelial cells [18]. A further direct mechanism of lung injury following bronchoaspiration may be subsequent to the induced surfactant dysfunction [19]. Besides these direct mechanisms, indirect pathways have also been reported to be involved in the course of aspiration, since the lung injury has been found to be mediated by capsaicin-sensitive vagal sensory afferent nerves [16, 20-22]. Another indirect route may be due to the activation of systemic inflammatory processes originating from the affected lungs, including the release of endogenous mediators by neutrophils, alveolar macrophages or by activation of the complement system leading to vascular leakage and oedema formation [16, 23-27]. Although the effective prevention and/or treatment of the bronchoaspiration would require the identification of the roles of these individual mechanisms, no previous studies have attempted to clarify the involvement of the direct and indirect pathways in the adverse functional changes in the lung during the acute phase of gastric juice inhalation. 
Another important factor that may affect the severity of the lung damage and the outcome of bronchoaspiration is the application of a raised positive end-expiratory pressure (PEEP). While an elevated PEEP is beneficial for the recruitment of lung regions and maintaining them open during mechanical ventilation [28, 29], a significant hemodynamic impairment characterized by a deterioration in cardiac function may also occur during a PEEP increment [30].

\subsection{Cardiopulmonary effects of blood loss and fluid replacement}

Perioperative morbidity may also be related to blood loss during major surgeries, which is associated with detrimental systemic and pulmonary consequences. Fluid replacement strategies under this condition are among the most polarizing issues in anaesthesia and intensive care practice. Physicians are routinely challenged with the choice of the best fluid replacement strategy for the treatment of haemorrhage from among blood products, various types of colloids or crystalloids. As an aftermath of the recent meta-analyses concerning the safety of hydroxyethyl-starch (HES) [31], this therapy should be considered with great caution, particularly in patients with increased capillary leakage. Thus, limited options are available for clinicians in fluid replacement strategies, in view of the risk of renal damage associated with the use of HES [32, 33], the appreciable costs of albumin, and the defects of haemostasis induced by gelatine solutions [34]. Crystalloids remain a rational option, but clinicians are reluctant to choose them because of the widespread belief of their fast extravasation, though this belief is based on old studies with limited evidence-based results [35-37].

It has been recently demonstrated that acute hypovolaemic shock and subsequent resuscitation with autologous blood affects the respiratory mechanics [38]. Although a milder, but sustained blood loss during a surgical procedure also requires fluid replacement therapy, the respiratory consequences of such a disorder have not been explored. The administration of blood products is often regarded as the gold standard therapy in this situation, with the main aim of maintaining the oxygen transport capacity. However, no evidence-based data are available that would allow a comparison of the changes in lung function between this consensual approach and goal-directed fluid therapy with colloids or crystalloids. 


\section{Aims}

The studies included in the present thesis were primarily aimed at examining the respiratory adverse effects of various anaesthesia-related complications, with particular focus on the characterization of the respiratory mechanical consequences of bronchoaspiration and blood loss and fluid replacement. The specific aims of the studies included in the present thesis were:

- to establish an animal model that allows separate investigation of the mechanical properties of the right and the left lung;

- to quantify the separate roles of direct and indirect mechanisms of the deterioration of lung mechanics following acid aspiration;

- to investigate the effects of different PEEP levels on the respiratory and circulatory outcomes during and following the inhalation of gastric juice;

- to develop a valid animal model which mimics continuous, hidden surgical bleeding and replacement of the lost blood; and

- to compare the effects of blood, colloid and crystalloid solutions on the flow resistance of the airways and on the viscoelastic properties of the respiratory tissues and to attempt to relate these changes to pulmonary oedema indices. 


\section{Methods}

Ethical approval for both studies was provided by the Experimental Ethics Committee of the University of Szeged, Szeged, Hungary (no. I-74-50/2012, Chairperson Prof. Gy. Szabó) on 7 December 2012, and granted by the Animal Health and Welfare Office of the local authorities in Hungary (no. XIV/152/2013, Chairperson Cs. Farle) on 9 January 2013.

\subsection{Animal preparations}

The choice of the animal models was based on the ability to intubate two lungs separately (porcine model) and the capability of comparing our data to the previously established literature (rats).

\subsubsection{Preparation of pigs}

Male Vietnamese mini-pigs $(\mathrm{n}=13)$ weighing $28.2 \pm 0.9 \mathrm{~kg}(21-33 \mathrm{~kg})$ were used in the present study. Anaesthesia was induced by an intramuscular injection of ketamine $(20 \mathrm{mg} / \mathrm{kg}$, CP-Ketamin $10 \%$, Produlab Pharma, Raamsdonksveer, Netherlands) and xylazine (2 mg/kg, CP-Xylazin $2 \%$, Produlab Pharma, Raamsdonksveer, Netherlands) until an i.v. line was secured into the ear. A continuous infusion of propofol $(6 \mathrm{mg} / \mathrm{kg} / \mathrm{h}$, Propofol $2 \% \mathrm{MCT} / \mathrm{LCT}$, Fresenius-Kabi, Bad Homburg, Germany) was then maintained throughout the study via the ear vein. The mini-pigs were tracheostomized and a double-lumen cannula (Broncho-cath right 35 Fr, Mallinckrodt Medical, Athlone, Ireland) was introduced into the distal trachea. The double lumen tracheostomy tube was positioned to achieve separate support of the left and right lung sides and the tips were sealed by inflating a cuff. The pigs were then mechanically ventilated (Model 900C; Siemens-Elema, Solna, Sweden) in volume-controlled mode with a tidal volume of $7-8 \mathrm{ml} / \mathrm{kg}$ at a frequency of $\sim 20 / \mathrm{min}$ and an inspired oxygen fraction $\left(\mathrm{FiO}_{2}\right)$ of 0.3 in air. A femoral artery was prepared surgically in a sterile manner and cannulated for blood sampling and the measurement of arterial blood pressure and cardiac output by thermodilution (PiCCO Catheters; PULSION Medical Systems, Feldkirchen, Germany). The jugular vein was prepared in the same way as the femoral artery and cannulated for fluid and drug administration. Muscle relaxation was achieved by regular i.v. administration of pipecuronium $(0.1 \mathrm{mg} / \mathrm{kg}$, every $30 \mathrm{~min}$, Arduan, Gedeon Richter, Budapest, Hungary). The thorax was opened by means of a midline thoracotomy following an 
i.v. bolus of sufentanyl $(25 \mu \mathrm{g} / \mathrm{kg}$, Sufentanil Torrex, Chiesi Pharmaceuticals, Vienna, Austria) and the ribs were widely retracted. Following chest opening, the pigs were randomly assigned to maintain a PEEP of 4 or $10 \mathrm{cmH}_{2} \mathrm{O}$.

Arterial blood samples were analysed radiometrically (Cobas b221; Roche Diagnostics, Basel, Switzerland). The Horowitz quotient (HQ) was calculated as the ratio of the arterial partial pressure of oxygen $\left(\mathrm{PaO}_{2}\right)$ and $\mathrm{FiO}_{2}$.

A small incision was made in the stomach and 10-20 ml of gastric juice was obtained via a catheter introduced into the stomach. This gastric juice was filtered to remove solid particles. The $\mathrm{pH}$ of the remaining fluid was determined and hydrochloric acid was added to reach a $\mathrm{pH}$ of 2 if needed.

\subsubsection{Preparation of rats}

Anaesthesia was induced with an intraperitoneal injection of $5 \%$ chloral hydrate $(400 \mathrm{mg} / \mathrm{kg})$ in adult male Sprague-Dawley rats $(\mathrm{n}=25,330 \pm 38 \mathrm{~g})$. Tracheal intubation was achieved with a polyethylene cannula (16-gauge, B. Braun Melsungen AG, Melsungen, Germany) after subcutaneous administration of local anaesthetics to ensure adequate analgesia around the surgical wound (lidocaine, $2-4 \mathrm{mg} / \mathrm{kg}$ ). The rats were then placed in a supine position on a heating pad and the tracheal cannula was attached to a small animal ventilator (Model 683, Harvard Apparatus, South Natick, MA, USA), and mechanically ventilated with room air ( 70 breaths $/ \mathrm{min}$, tidal volume $7 \mathrm{ml} / \mathrm{kg}$ ). A femoral vein was catheterized (Abocath $22 \mathrm{G}$ ) for drug delivery and for the fluid replacement. A femoral artery was cannulated (Abocath $22 \mathrm{G}$ ) and attached to a pressure transducer (Model TSD104A, Biopac, Santa Barbara, CA, USA) for continuous systemic blood pressure monitoring to assess mean arterial pressure (MAP), and to allow blood withdrawal, as part of the experimental protocol. The arterial pressure, ECG and heart rate (HR) were monitored continuously with a data collection and acquisition system (Biopac, Santa Barbara, CA, USA). Body temperature was kept in the $37 \pm 0.5^{\circ} \mathrm{C}$ range by using the heating pad. 


\subsection{Measurement of respiratory mechanics}

\subsubsection{Forced oscillatory measurements in pigs}

The measurement system for collection of the input impedance spectra of the right or left lung $\left(\mathrm{Z}_{\mathrm{L}, \mathrm{s}}\right)$ in the mini-pigs was similar to that can be used for whole lungs [39]. Briefly, the mechanical ventilation was ceased at end-expiration and the cannula of the measured lung was connected to a loudspeaker-in-box system while the cannula of the other lung was occluded as depicted in Figure 1 by means of two clamps: one on the tubing of the ventilator circuit and one on the opposite lumen of the double lumen endotracheal (ET) tube. Thus, during these 8-s long apnoeic periods, the forced oscillatory signal was introduced only to either the left or the right lung. Prior to the measurements, the pressure in the loudspeaker box chambers was set to the level of the PEEP to keep the mean transpulmonary pressure constant. The loudspeaker delivered a computer-generated small-amplitude $\left(< \pm 1 \mathrm{cmH}_{2} \mathrm{O}\right)$ pseudo-random signal composed of 23 non-integer multiples between 0.5 and $21 \mathrm{~Hz}$ with preset amplitudes and phases through a screen pneumotachograph (11 $\mathrm{mm}$ internal diameter), which was used to measure the gas flow $(\dot{V})$ with a differential pressure transducer (model 33NA002D; ICSensors, Milpitas, CA, USA). An identical pressure transducer was used to measure the pressure in the left or right main bronchi with reference to the atmosphere $\left(\mathrm{P}_{\mathrm{L}, \mathrm{s}}\right)$.

The $\mathrm{P}_{\mathrm{L}, \mathrm{s}}$ and $\mathrm{V}$ signals were low-pass filtered at $25 \mathrm{~Hz}$ and sampled with an analogue-digital board of a microcomputer at a rate of $256 \mathrm{~Hz}$. Fast Fourier transformation with 4-s time windows and $95 \%$ overlapping was used to calculate the unilateral $\mathrm{Z}_{\mathrm{L}, \mathrm{s}}$ spectra:

$$
Z_{L, S}=\frac{P_{L, S}}{\dot{V}}
$$

The notations $\mathrm{P}_{\mathrm{L}, \mathrm{s}}$ and $\mathrm{V}$ in the above equation relate to the power spectra of the pressure and flow signals, respectively, allowing frequency-domain analysis of the impedance spectra. 


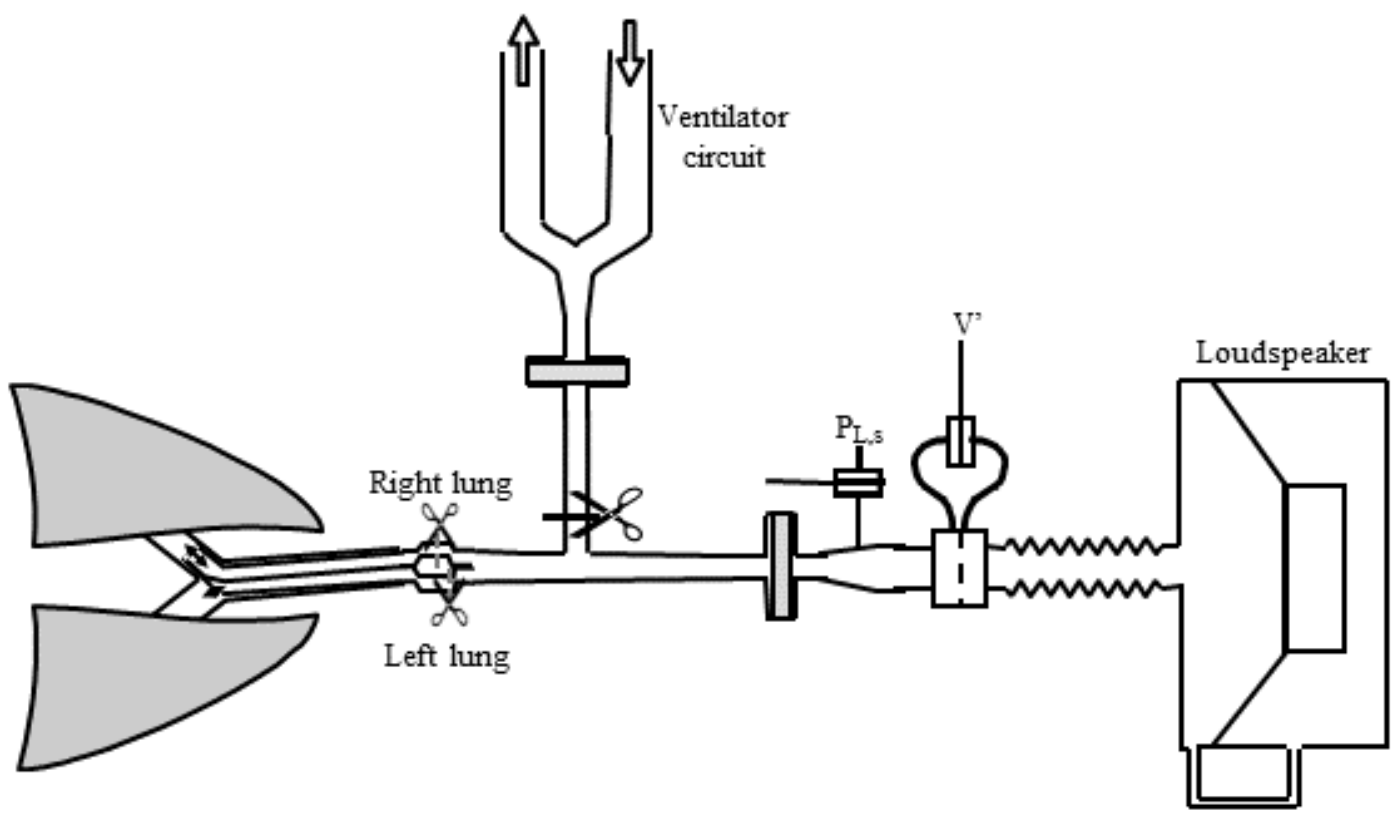

Figure 1. Experimental set-up for lung measurement in pigs.

During measurements, the ventilator circuit and the appropriate lumen of the doublelumen tube are clamped at the sites indicated by scissor symbols. $P_{L, s}$ : pressure transducer, $V$ ': flow transducer.

\subsubsection{Forced oscillatory measurements in rats}

Since airflow measurement cannot be performed reliably in small tubing, the wave-tube technique was applied in the rat experiments. The forced oscillation technique in this species was applied in short (6-s-long) end-expiratory pauses interposed in the mechanical ventilation to measure the input impedance of the respiratory system $\left(\mathrm{Z}_{\mathrm{rs}}\right)$, as detailed previously [40] and depicted in Figure 2. Briefly, the ventilator was stopped at end-expiration and the tracheal cannula was switched from the ventilator to a loudspeaker-in-box system by means of a threeway tap. The loudspeaker delivered a computer-generated small-amplitude $\left(< \pm 1 \mathrm{cmH}_{2} \mathrm{O}\right)$ pseudorandom signal through a 100-cm-long, 2-mm internal diameter polyethylene tube. Two identical pressure transducers (model 33NA002D, ICSensors, Milpitas, CA, USA) were used to measure the lateral pressures at the loudspeaker end $\left(\mathrm{P}_{1}\right)$ and at the tracheal end $\left(\mathrm{P}_{2}\right)$ of the wave-tube. The signals $\mathrm{P}_{1}$ and $\mathrm{P}_{2}$ were low-pass filtered (5th-order Butterworth, 25-Hz corner frequency), and sampled with the analogue-digital board of a microcomputer at a rate of 
$256 \mathrm{~Hz}$. Fast Fourier transformation with 4-s time windows and $95 \%$ overlapping was used to calculate the pressure transfer functions $\left(\mathrm{P}_{1} / \mathrm{P}_{2}\right)$ from the 6-s recordings collected during apnoea. $\mathrm{Z}_{\mathrm{rs}}$ was calculated as the load impedance of the wave-tube [41].

$$
Z_{r s}=\frac{Z_{0} \cdot \sinh (\gamma L)}{\frac{P_{1}}{P_{2}}-\cosh (\gamma L)}
$$

In the above equation $\mathrm{P}_{1}$ and $\mathrm{P}_{2}$ represent the power spectra of the pressure signals, with $\mathrm{P}_{1} / \mathrm{P}_{2}$ as the pressure transfer function of the wave-tube, $\mathrm{Z}_{0}$ is the characteristic impedance calculated from the geometrical, mechanical and thermodynamic properties of the wave-tube and the filling gas (air), L represents the length of the tube and $\gamma$ is the complex wave propagation number.

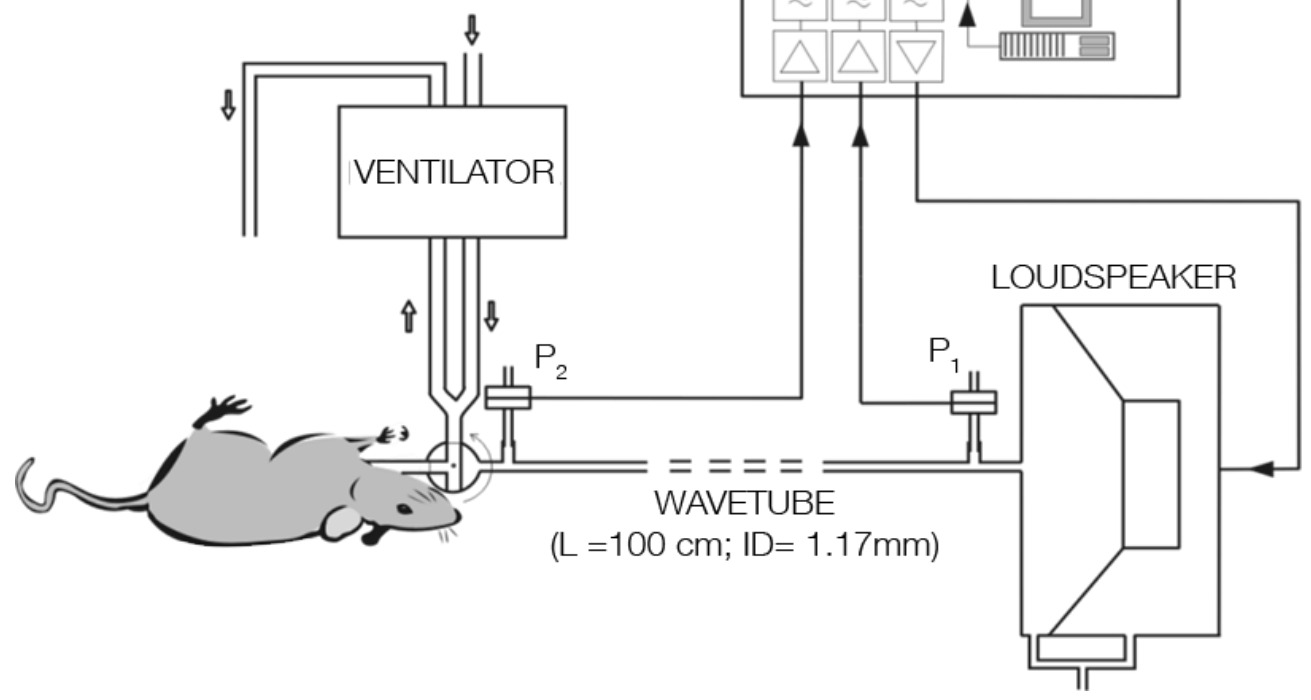

Figure 2. $\quad$ Experimental set-up for lung measurements in rats.

During normal ventilation the three-way tap connects the animal and the ventilator circuit, while during measurements the animal and the loudspeaker are connected. $P_{1}$ and $P_{2}$ : pressures at the loudspeaker and the tracheal end of the wave-tube, respectively.

\subsubsection{Calculation of mechanical properties from the respiratory impedance spectra}

The input impedance of the ET tube and the connective tubing was determined in both studies and was subtracted from each $\mathrm{Z}_{\mathrm{rs}}$ or $\mathrm{Z}_{\mathrm{L}, \mathrm{s}}$ spectrum. 
The airway and parenchymal mechanical properties for the individual lungs of the pigs or respiratory systems of the rats were separated by fitting a model to the $\mathrm{Z}_{\mathrm{L}, \mathrm{s}}$ or $\mathrm{Z}_{\mathrm{rs}}$ spectra $(\mathrm{Z})$ by minimizing the weighted differences between the measured and modelled impedance values. The model consisted of an airway compartment containing a frequency-independent airway resistance (Raw) and airway inertance (Iaw), and a constant-phase tissue unit [42] characterized by tissue damping $(\mathrm{G})$ and elastance $(\mathrm{H})$ :

$$
Z=\operatorname{Raw}\left(1+\beta \omega^{2}\right)+j \omega I a w+\frac{(G-j H)}{\omega^{\alpha}}
$$

where $j$ is the imaginary unit, $\omega$ is the angular frequency $(2 \pi f), \alpha$ is $(2 / \pi) \arctan (H / G)$ and $\beta$ is an empirical parameter [43], allowing the model fitting of spectra with slightly elevating highfrequency components in the real parts of the porcine measurements. In the rat study, the value of $\beta$ was fixed to 0 , since no elevation was observed in the real parts of the spectra.

The tissue parameters $\mathrm{G}$ and $\mathrm{H}$ are attributed to the damping (resistive) and elastic properties of the respiratory system. Raw and Iaw represent primarily the resistance and inertance of the major conducting airways. Tissue damping is attributed to the energy loss of the respiratory tissues from internal friction, with higher values associated with increased heterogeneities or ventilation-perfusion mismatch. Tissue elastance, as the opposite of compliance, is attributed to the ability of the lung to recoil to its normal state. Higher values indicate a stiffer lung, therefore a lower compliance. In pigs as the thorax was opened and widely retracted, the recorded impedance and calculated parameters are related to the mechanical parameters of the individual lungs. In rats where the chest is kept intact, tissue parameters are influenced by the mechanical properties of the chest wall itself, while the contribution of the chest wall to resistance and inertance in rats is minor [44].

\subsection{Additional measurements}

\subsubsection{Measurement of exhaled carbon-dioxide in pigs}

Changes in partial $\mathrm{CO}_{2}$ pressure in the exhaled gas during mechanical ventilation were measured with a calibrated sidestream capnograph (Cardiocap II; Datex/Instrumentarium, Helsinki, Finland). $\mathrm{CO}_{2}$ traces were recorded alternately from each lung by closing one lumen of the endotracheal tube, at a time while the tidal volume was decreased by one-third by elevating the ventilation frequency. The 8-s $\mathrm{CO}_{2}$ traces obtained from the aspirated or the 
intact lung were imported into a custom-developed signal analysis software. The third phase of the expiratory $\mathrm{CO}_{2}$ curves in each expiration was identified by selecting the $\mathrm{CO}_{2}$ traces from its peak end-tidal value back to $60 \%$ of the total length of its linear temporal change. Linear regression analysis was applied to these phases to obtain slope of the third phase of the expiratory capnogram $\left(\mathrm{S}_{\mathrm{III}}\right)$. $\mathrm{S}_{\mathrm{III}}$ was normalized by dividing each slope by the average values of the corresponding $\mathrm{CO}_{2}$ concentration in the mixed expired gas to obtain normalized time domain third phase slopes $\left(\mathrm{S}_{\mathrm{nIII}}\right)$. Four to six expiratory traces were analysed in each recording.

\subsubsection{Haemodynamic monitoring of pigs}

Systemic haemodynamic parameters were monitored by a transpulmonary arterial thermodilution system (PiCCO; Pulsion Medical System, Munich, Germany). A 4F arterial catheter was inserted into the femoral artery. The thermal indicator bolus was injected into the right atrium via a central venous catheter positioned in the jugular vein. The monitor determined the cardiac index (CI) by normalizing the cardiac output to the body surface area. The same monitoring system was used to measure the mean arterial pressure (MAP) and heart rate (HR).

\subsubsection{Lung histology in rats}

After completion of the experimental protocol, the rats were euthanized with an overdose of pentobarbital sodium (300 mg/kg iv). Midline thoracotomy was then performed and $4 \%$ formaldehyde was instilled into the right lung via the tracheal cannula at a hydrostatic pressure of $20 \mathrm{cmH}_{2} \mathrm{O}$ after clamping of the left main bronchus near the bifurcation. The right lung was dissected and placed into $4 \%$ buffered formalin until further processing. After complete fixation, transhilar horizontal sections (perpendicular to the longitudinal axes of the lung from the hilum) were embedded in paraffin. Two 5- $\mu$ m sections were prepared in each lung specimen and were stained with haematoxylin-eosin. Digitalized images were used to obtain the oedema index around randomly selected pulmonary vessels by dividing the lumen area by the total area of the pulmonary vessel (oedema cuff area + vessel lumen area). Histological images were analysed by the same investigator in a blind fashion and in a random sequence by using JMicroVision image analysis software (version 1.2.7). 
Three-to-four tissue samples were dissected from the different lobes of the non-fixated left lungs; these samples were weighed to establish the wet-to-dry weight ratio (W/D) as an index of the lung water content.

\subsection{Experimental protocols}

\subsubsection{Characterization of effects of unilateral aspiration in pigs}

Following stabilization of the pulmonary mechanical and haemodynamic variables after the anaesthesia and the preparation, the pigs were randomly assigned to groups in which the PEEP was set to a level of either 4 (Group P4, $\mathrm{n}=7$ ) or $10 \mathrm{cmH}_{2} \mathrm{O}($ Group P10, $\mathrm{n}=6$ ). Two sets of baseline $\mathrm{Z}_{\mathrm{L}, \mathrm{s}}$ recordings were then collected for each lung separately by forced oscillations (Figure 3). This was followed by registration of the $\mathrm{CO}_{2}$ traces from each lung separately. Aspiration was achieved by unilaterally instilling the prepared gastric juice mixed with hydrochloric acid ( $\mathrm{pH}$ 2) into the endobronchial lumen (right lung) of the tracheal tube $(0.5 \mathrm{ml} / \mathrm{kg})$ and leaving the left lung unaffected. To maintain the best possible gas exchange and similarity to a clinical situation, $\mathrm{FiO}_{2}$ was elevated to 1 after injection of the gastric content. Forced oscillatory and capnogram measurements were recorded for each lung alternately at 5, 15, 30, 45 and 60 min following aspiration. To assess the effect of a PEEP change in this period, the level of PEEP was interchanged between the groups (10 to $4 \mathrm{cmH}_{2} \mathrm{O}$ and 4 to $10 \mathrm{cmH}_{2} \mathrm{O}$, respectively) $60 \mathrm{~min}$ post-aspiration. Following this PEEP interchange, additional $\mathrm{Z}_{\mathrm{L}, \mathrm{s}}$ and $\mathrm{CO}_{2}$ recordings were collected at 65,75 and $90 \mathrm{~min}$ post-aspiration. Finally, PEEP was re-established at the original level and additional measurements were performed at 95, 105 and 120 min post-aspiration. Blood gas samples were analysed and thermodilution measurements were performed at baseline and at 20, 80 and $110 \mathrm{~min}$ postaspiration. 


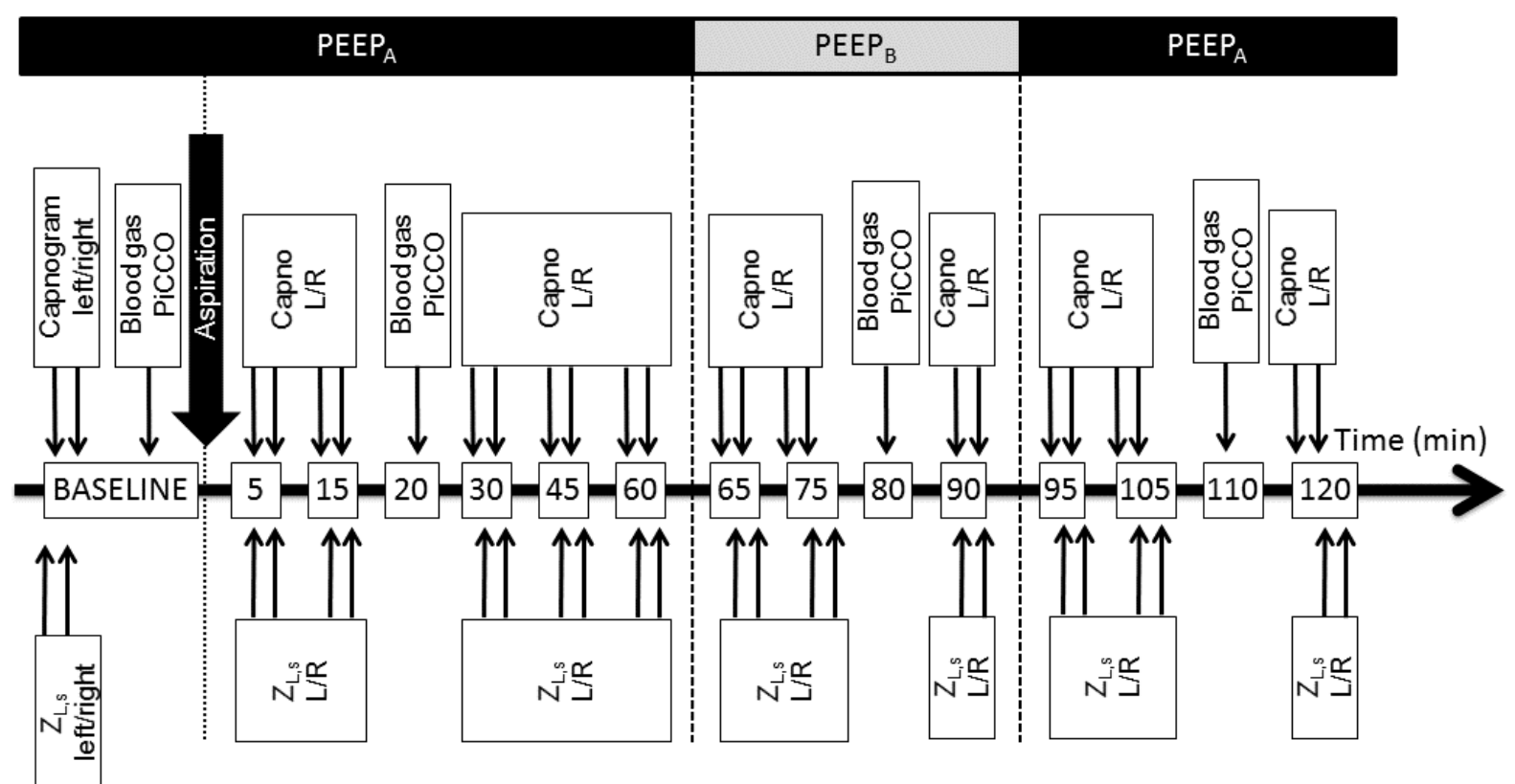

Figure 3. $\quad$ Scheme of the experimental protocol in pigs.

$Z_{L, s}:$ unilateral pulmonary input impedance measurement. L/R: measurement of left or right lung. $P E E P_{A}$ and $P E E P_{B}$ correspond to 4 and $10 \mathrm{cmH}_{2} \mathrm{O}$, respectively, in random sequence.

\subsubsection{Assessment of respiratory effects of blood loss and fluid replacement in rats}

The rats were randomly assigned into one or other of the three protocol groups. The rats in Group B always received autologous heparinized blood $(n=8)$, while fluid replacement was performed with a colloid solution (HES $6 \%$ 130/0.4, Fresenius Kabi Deutschland GmbH, Bad Homburg v.d.H., Germany) in Group $\mathrm{CO}(\mathrm{n}=8)$, or with a crystalloid solution $(\mathrm{NaCl}$ $0.9 \%$, B. Braun Melsungen AG, Melsungen, Germany) in Group CR $(n=9)$. The experimental protocol was started with standardization of the lung volume history through the administration of a hyperinflation via occlusion of the expiratory port of the ventilator when the animal had reached a steady-state condition (5-10 min after the starting of mechanical ventilation). The baseline respiratory mechanics was then established by measuring 3 or 4 reproducible $\mathrm{Z}_{\mathrm{rs}}$ data epochs. Haemorrhage was next induced by the withdrawal of $5 \%$ of the estimated total blood volume [45] via the femoral artery (Figure 4). Three minutes later, another set of $Z_{\mathrm{rs}}$ data was collected, including 3 individual measurements at 1-min intervals. The withdrawn blood was used for blood gas analyses (Cobas b221; Roche Diagnostics, Basel, Switzerland) to determine the haematocrit (Hct), $\mathrm{pH}$ and oxygen $\left(\mathrm{PaO}_{2}\right)$ and carbon 
dioxide $\left(\mathrm{PaCO}_{2}\right)$ partial pressures. The blood withdrawal and $\mathrm{Z}_{\mathrm{rs}}$ measurements were repeated once again in an identical manner. After completion of the first two steps of arterial haemorrhage, fluid replacement in accordance with the group allocations was performed by administering $5 \%$ of the total blood volume via the femoral vein. Three minutes after this manoeuvre, a set of $Z_{\mathrm{rs}}$ data was recorded. The blood withdrawal-replacement procedure was repeated 4 more times, with the collection of $\mathrm{Z}_{\mathrm{rs}}$ data $3 \mathrm{~min}$ after each intervention. The total duration of resuscitation was around $90 \mathrm{~min}$ with each step lasting approximately $7 \mathrm{~min}$. Further arterial blood gas analyses were performed from the fourth and sixth blood samples. After completion of the measurement protocol, the lungs were processed for oedema assessment, as detailed above.

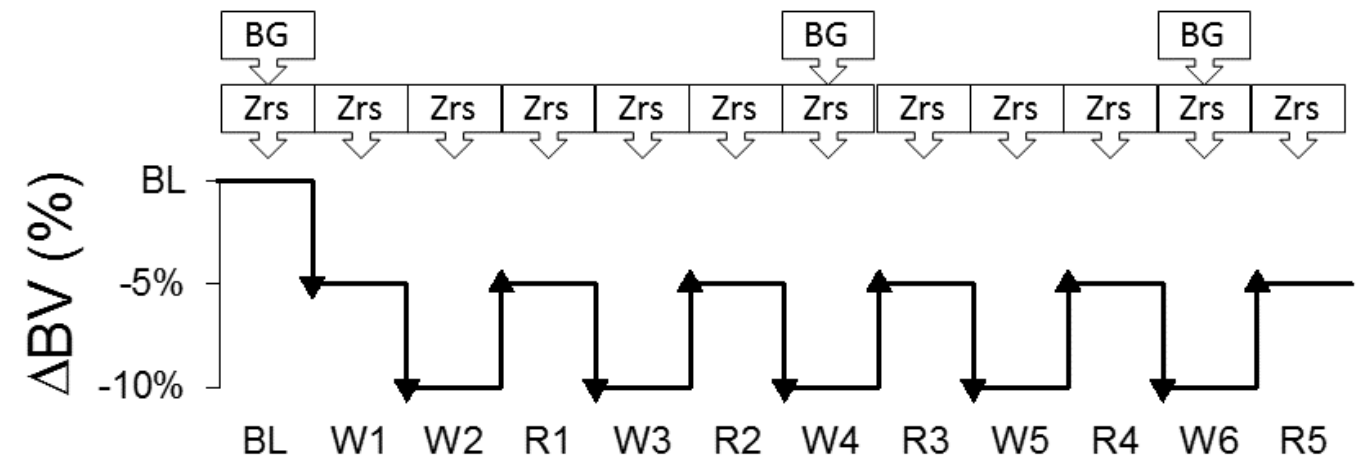

Figure 4. Scheme of the experimental protocol in rats.

$\triangle B V$ : Change in total blood volume. BL: baseline, W1-W6: blood withdrawals, R1-R5: fluid replacements, Zrs: measurement of respiratory impedance data, BG: assessment of arterial blood gas.

\subsection{Statistical analyses}

The scatters in the parameters were expressed as SEM values. The Kolmogorov-Smirnov test was used to test data for normality.

In pigs, two-way repeated measures mixed model of analysis of variance (ANOVA) with the factors assessment time (control vs. post-aspiration) and lung side (aspirated or intact) was used in both PEEP-based groups to assess the effects of the aspiration of the gastric contents on the lung responsiveness, the slope of the third phase of the capnogram and on haemodynamic parameters. The choice of appropriate covariance structure was on the basis of 
Akaike information criterion measure. The Holm-Sidak multiple comparison procedure was applied to compare the different conditions (for repeated measures) or lung sides (affected or intact). Correlation analyses between the variables were performed by using Pearson correlation tests.

In rats, two-way repeated measures ANOVA tests with the factors assessment time and group allocation were used to assess the effects of blood loss and replacement on the respiratory mechanical and haemodynamic parameters. The baseline respiratory mechanical parameters and oedema indices were compared by using one-way ANOVA tests. The Holm-Sidak multiple comparison procedure was applied to compare the different conditions (for repeated measures) or protocol groups (for independent groups). Correlation analyses between the variables were performed by using Pearson correlation tests.

Statistical tests were carried out with the SigmaPlot software package (version 12.5, Systat Software, Inc, CA, USA) and SAS (version 9.2, SAS Institute, Cary, NC, USA) with a significance level of $\mathrm{p}<0.05$. 


\section{Results}

\subsection{Pulmonary consequences of bronchoaspiration: results in pigs}

There was no statistically significant difference between the weights of the pigs in the two protocol groups $(29.6 \pm 1.1$ vs. $26.7 \pm 1.4 \mathrm{~kg}$ for Groups $\mathrm{P} 4$ and P10, respectively, $\mathrm{p}=0.125)$. The quality of the fitting of the model to the $\mathrm{Z}_{\mathrm{L}, \mathrm{s}}$ spectra was equally good in each group and experimental condition with mean fitting error of $6.1 \pm 0.12 \%$.

\subsubsection{Qualitative changes of the impedance spectra}

Affected lung
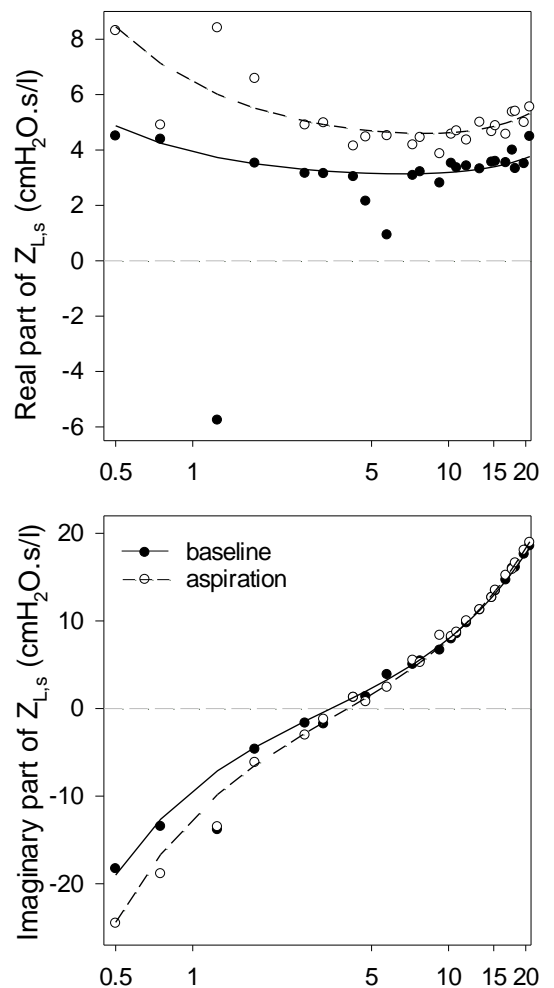

Intact lung
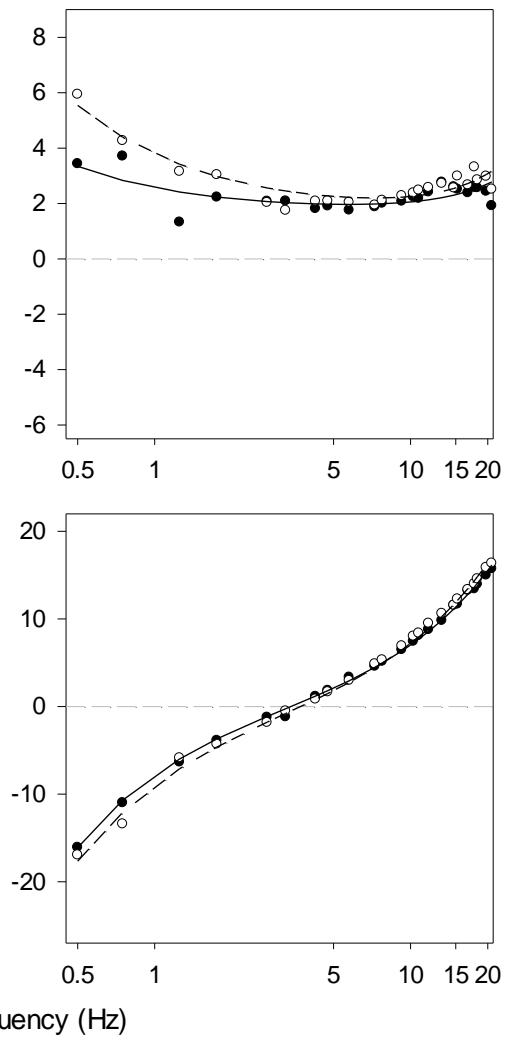

Figure 5. Impedance spectra from a representative pig.

Real (upper panels) and imaginary parts (lower panels) are presented. The left panels exhibit spectra from the affected lungs, while the right panels are spectra of the intact lungs. Symbols denote the actual measured spectra, while lines indicate the model fitted impedances. Continuous lines and filled symbols relate to the baseline spectra, while dashed lines and empty symbols show spectra following aspiration. Note the logarithmic scale of the frequency axis. 
Changes in the impedance spectra in a representative pig are presented in Figure 5. The impedances of the affected and the intact sides are depicted separately, as functions of frequency. The characteristic frequency-dependence of the impedance spectra can be observed in the baseline curves: the real part decreases with increasing frequency, with a slight increase towards the end of the frequency range studied, while the imaginary part increases monotonously with frequency. A few outlying points can be observed in both curves representing the real and imaginary parts of the impedance data (e.g. the third point of the affected real and imaginary curves under the baseline), which vary greatly with repeated measurements. These points occur at frequency values corresponding to the heart rate and its upper harmonics and are not related to the respiratory system itself, but are artefacts related to cardiac activity. They are therefore omitted from model fitting.

The parameters of the fitted model can be approximately attributed to certain sections of the impedance spectra. The quasi-straight section of the real part at higher frequencies is mainly related to Raw, while the low-frequency section of the real part is related to G. The lowfrequency section of the imaginary part is related to $\mathrm{H}$, and the higher-frequency components of the imaginary part are related to Iaw.

It can be observed from the Figure that the baseline spectra of the affected and intact sides are qualitatively similar, while the responses to aspiration differ. Changes were observed in the affected lung with an upward shift in the real part (elevations in Raw and G), associated with a downwards shift in the low-frequency section of the imaginary part (elevation in $\mathrm{H}$ ). In the intact lung, no such changes were obvious.

\subsubsection{Unilateral lung mechanical changes}

Figure 6 demonstrates the temporal changes in the airway and lung tissue forced oscillatory mechanical parameters in both experimental groups for the affected (aspirated) and intact lungs separately. The mechanical changes following aspiration appeared generally greater in Group P4 than in P10. Under the baseline conditions, the mechanical parameters did not exhibit any statistically significant differences between the two lung sides in either experimental group. However, in both groups of pigs, significant differences developed between the two sides following gastric juice aspiration: in Raw (at least $\mathrm{p}<0.018$ and $\mathrm{p}<0.032$ for Groups P4 and P10, respectively), G $(\mathrm{p}<0.05, \mathrm{p}<0.05)$ and $\mathrm{H}(\mathrm{p}<0.05$, 
$\mathrm{p}<0.036$ ). The peak of the mechanical deterioration on the aspirated side occurred 5-15 min after the bronchoaspiration, with gradual improvements thereafter. The airway and tissue mechanical parameters for the intact side did not exhibit any significant change throughout the study protocol.

The changes in the lung mechanical parameters relative to their baseline values are depicted in Figure 7 for characteristic time points in the experiments. On the aspirated side, the airway and lung tissue mechanical indices were increased significantly 5 min after the aspiration in both Group P4 ( $p<0.001, p=0.001$ and $p<0.001$ for Raw, $\mathrm{G}$ and $\mathrm{H}$, respectively) and Group P10 $(\mathrm{p}=0.012, \mathrm{p}=0.007$ and $\mathrm{p}<0.001)$. The changes on the aspirated side were greater than those on the intact side 5 min after the aspiration in the pigs in Groups P4 $(\mathrm{p}<0.014)$ and P10 ( $<0.037)$; significant differences were also apparent later for Raw and H in Group P4 ( $<<0.008, p=0.029)$ and for G and H in Group P10 ( $p=0.037, p<0.009)$. Significant recoveries started from $60 \mathrm{~min}$ after the aspiration in all mechanical parameters. Lowering the PEEP to $4 \mathrm{cmH}_{2} \mathrm{O}$ in Group $\mathrm{P} 10$ caused a significant decrease in $\mathrm{H}(\mathrm{p}=0.002)$ on the intact side, whereas elevating the PEEP in Group P4 did not have a major effect on the unilateral lung mechanics. The unilateral lung mechanical parameters for the intact side did not demonstrate any significant adverse change following aspiration; in Group P10, G and $\mathrm{H}$ were even decreased on the unaffected side 120 min after aspiration $(p=0.021, p=0.022)$. Since indirect mechanisms are expected to alter primarily the airway tone, the changes in Raw in the two lungs were related to reveal the relationship between the magnitudes of the bronchoconstriction. Figure 8 demonstrates the associations between the aspiration-induced changes in Raw obtained from the intact and aspirated sides for the first $60 \mathrm{~min}$ of the experiment. While fairly systematic elevations were observed in Raw on the aspirated side, the changes on the intact side were highly variable, with the majority of the pigs exhibiting even decrements. Nevertheless, there was a statistically significant correlation between the Raw changes on the aspirated and intact sides $(R=0.41, p=0.002)$. 

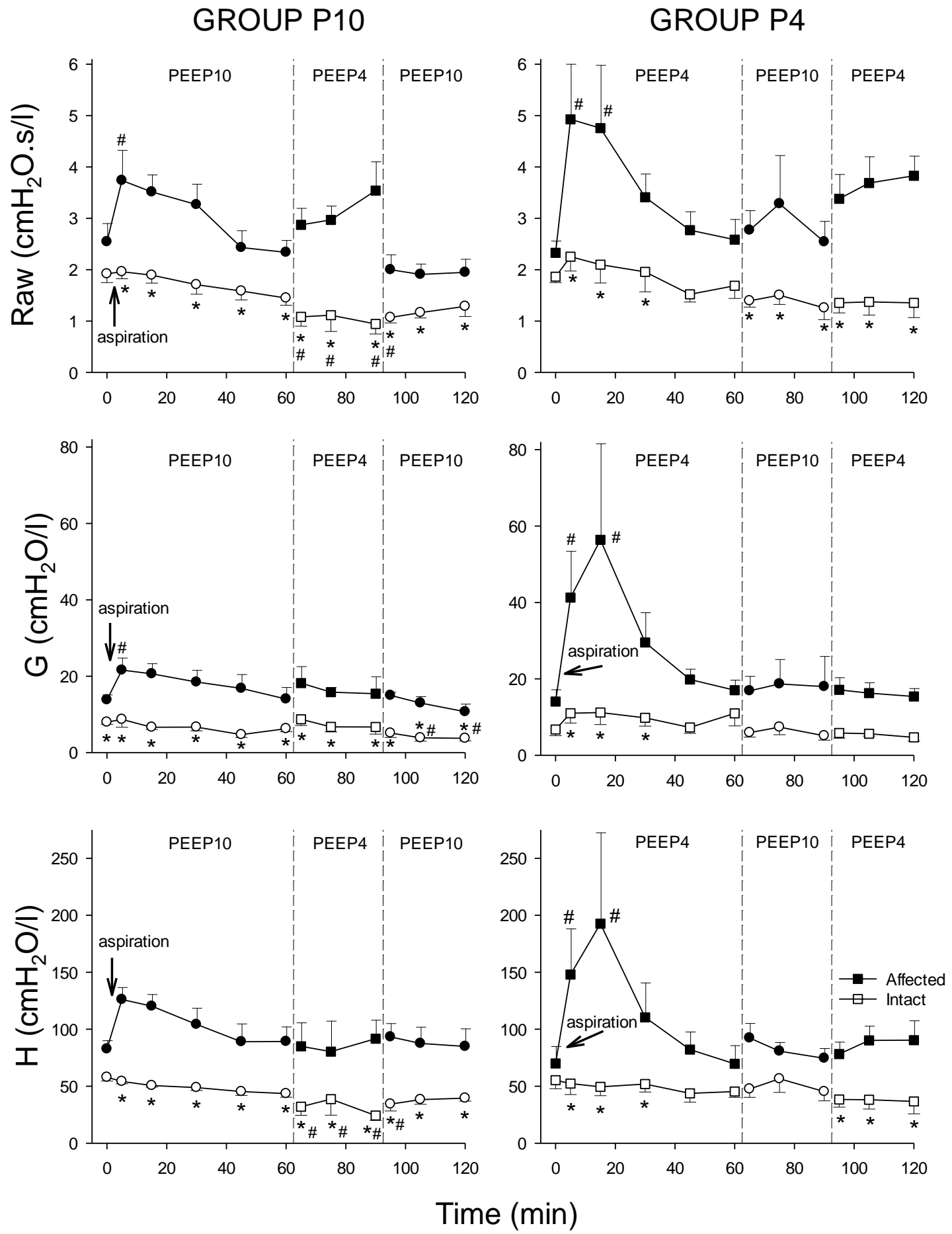

Figure 6. $\quad$ Airway resistance (Raw), tissue damping $(G)$ and elastance $(H)$ for the affected and the intact lungs in Groups P10 and P4 under baseline conditions (0 min) and following aspiration.

$*: p<0.05$ between affected and intact lungs; ${ }^{*}: p<0.05$ vs. baseline conditions within the same lung. 

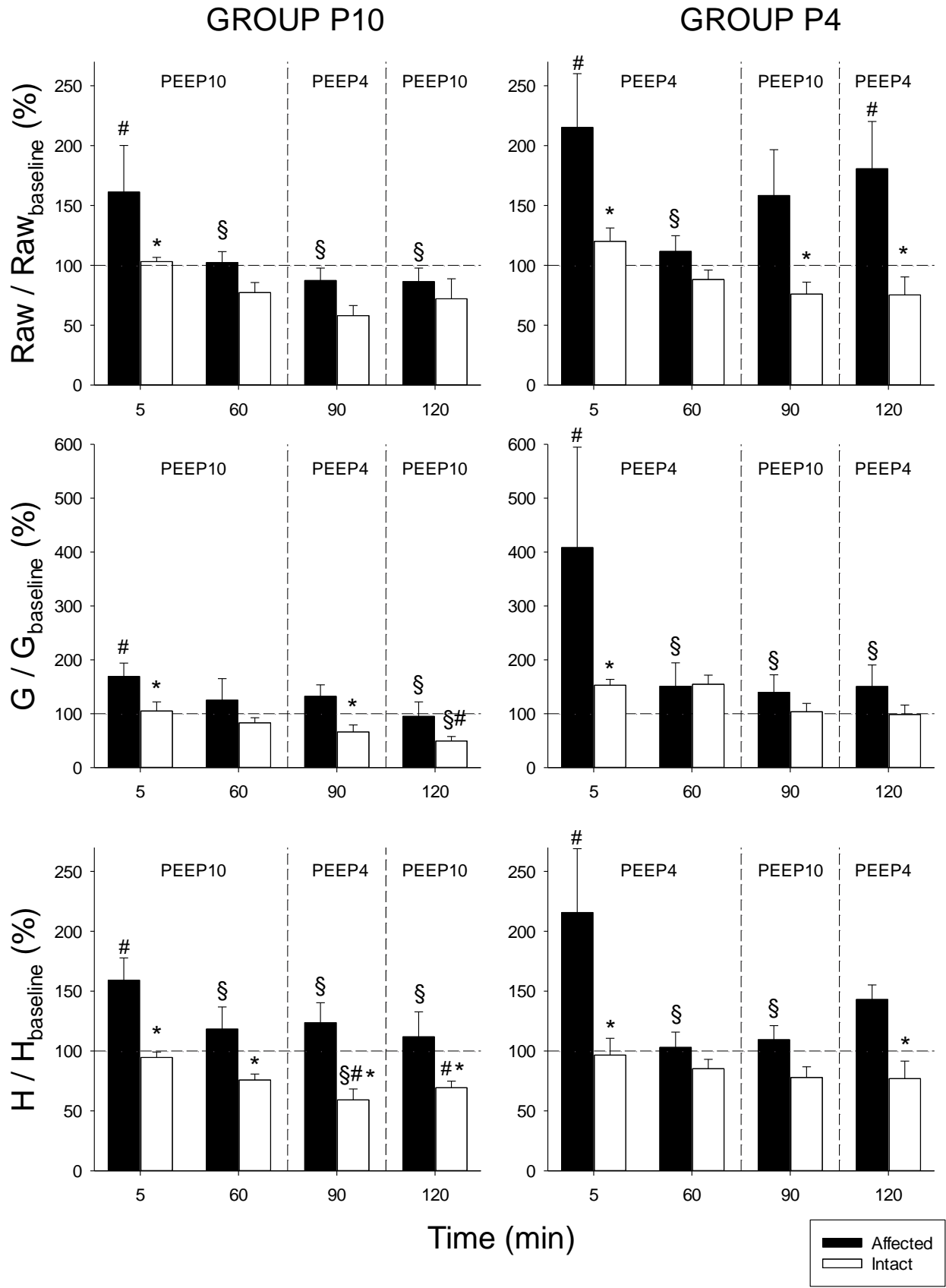

Figure 7. Relative changes in airway resistance (Raw), tissue damping $(G)$ and elastance $(H)$ in response to bronchoaspiration at 5, 60, 90 and $120 \mathrm{~min}$.

$*: p<0.05$ between affected and intact lungs; ${ }^{*}: p<0.05$ vs. baseline conditions within the same lung; ${ }^{\xi}: p<0.05$ vs. values at 5 min within the same lung. 


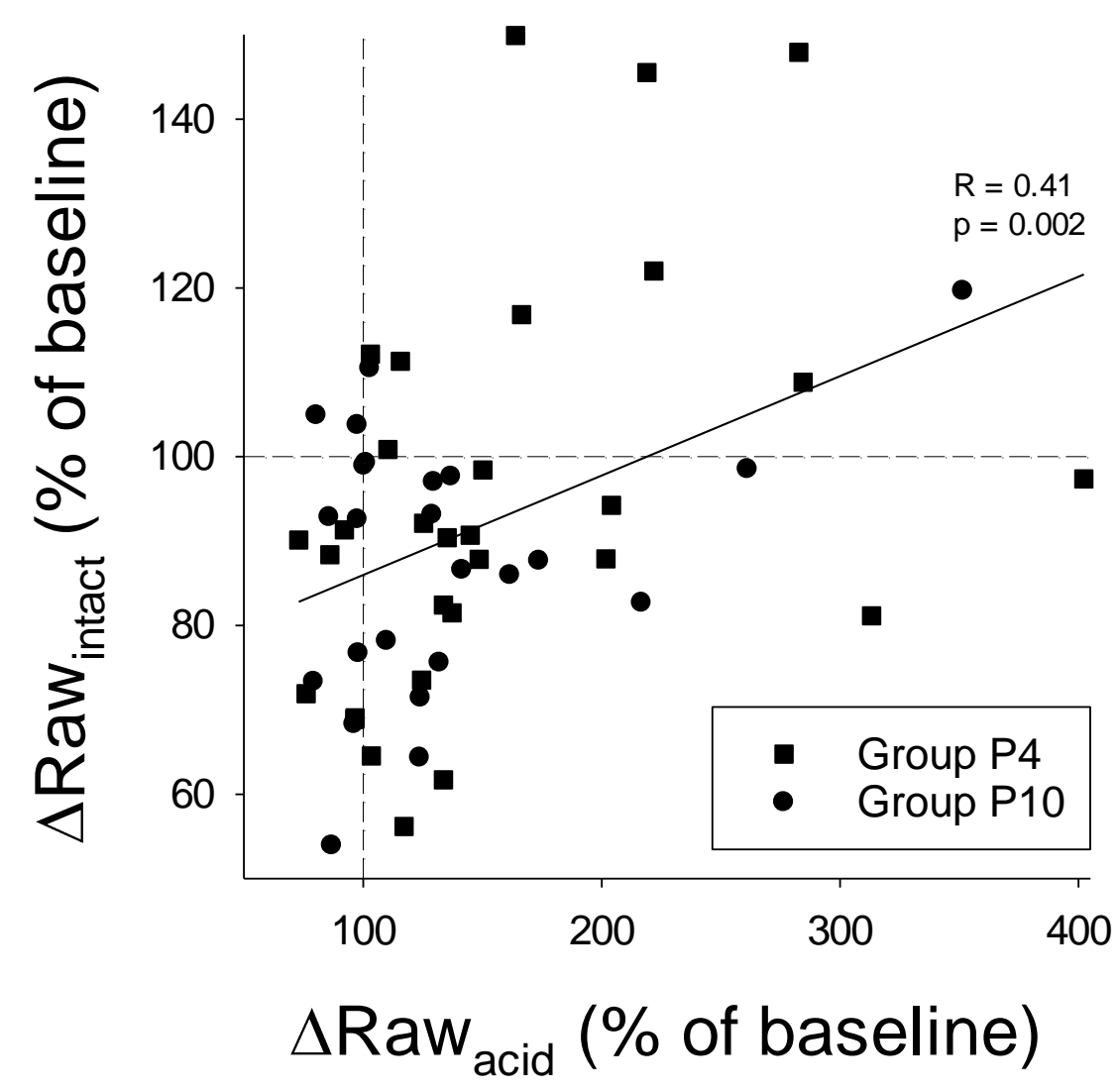

Figure 8. Association between the changes in airway resistance in the affected and the intact lung in the first 60 min following aspiration in Groups P4 and P1O.

Solid line: linear regression.

\subsubsection{Changes in the capnogram parameters}

The changes in the absolute and normalized third phase slope of the capnogram are demonstrated in Figure 9 under baseline conditions and 5, 60, 90 and $120 \mathrm{~min}$ after bronchoaspiration. No significant change was detected in $S_{\text {III }}$ in Group P10 throughout the study, whereas $S_{\text {III }}$ was elevated at 5 min on the aspirated side in Group P4 ( $\left.p=0.01\right)$, with an apparent recovery at $120 \mathrm{~min}(\mathrm{p}=0.044) . \mathrm{S}_{\mathrm{nIII}}$ did not change on the intact side during the study, whereas it was significantly elevated on the aspirated side in both groups 5 min after the aspiration ( $\mathrm{p}=0.021$ and $\mathrm{p}=0.001$ for Group P10 and P4, respectively). Changing the PEEP elevated the aspirated-side $S_{\mathrm{nIII}}$ in the pigs of Group P10 ( $\left.\mathrm{p}=0.007\right)$, while there was no change in the intact side in the pigs of Group P10, or on either side in Group P4. 


\section{GROUP P10}
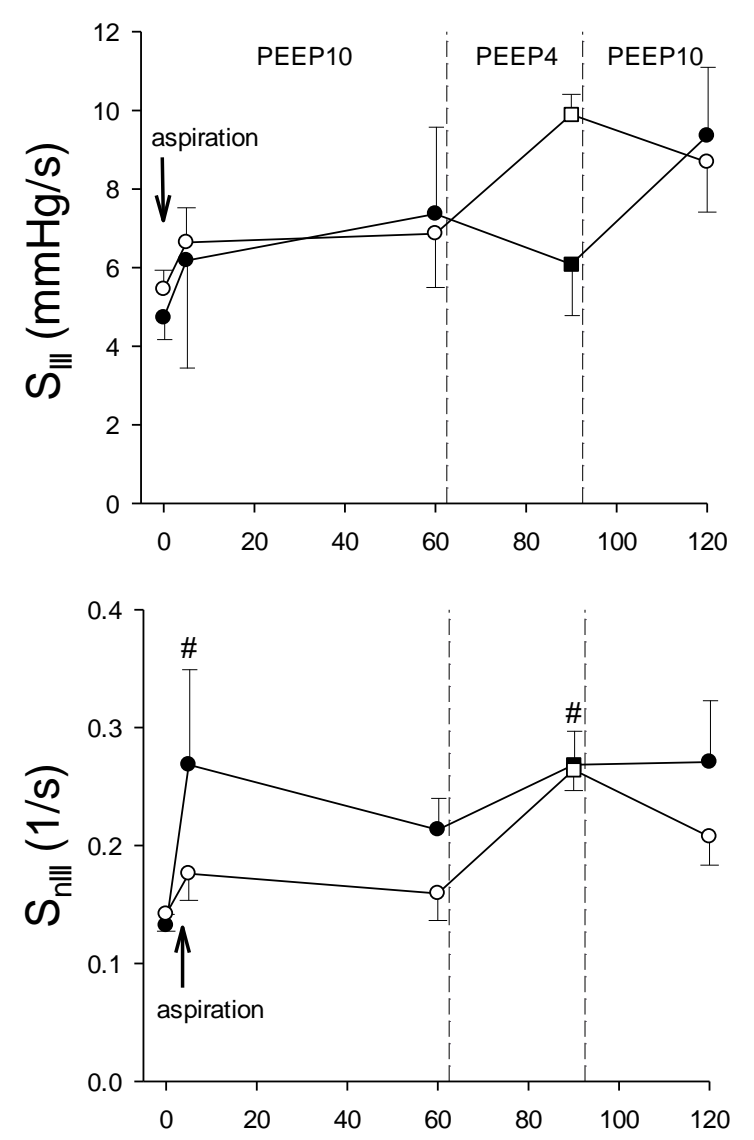

\section{GROUP P4}
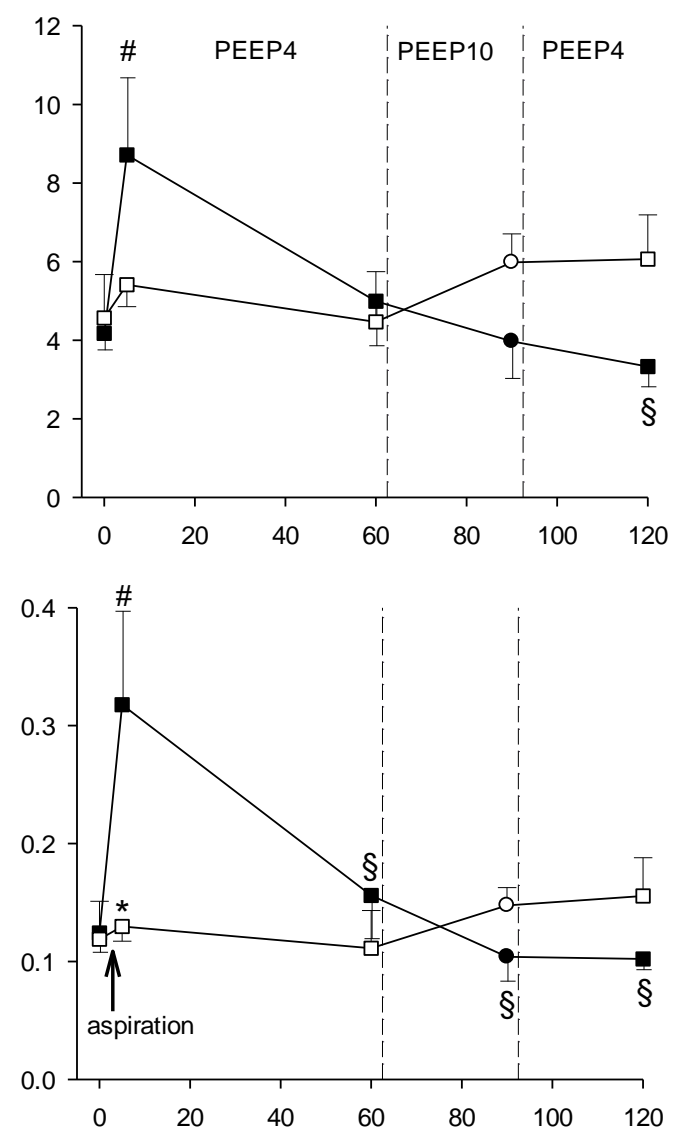

Time (min)

Figure 9. Third phase slope of the capnogram $\left(S_{I I I}\right)$ and its normalized value $\left(S_{\text {nIII }}\right)$ for the affected and the intact lungs in Groups P10 and P4 under baseline conditions (0 min) and following aspiration.

$*: p<0.05$ between affected and intact lungs; ${ }^{*}: p<0.05$ vs. baseline conditions within the same lung; ${ }^{\S}: p<0.05$ vs. values at 5 min within the same lung.

\subsubsection{Haemodynamic changes}

Under the baseline conditions, CI and HR did not differ between the protocol groups, while the MAP in Group P10 was lower than that in Group P4 $(81.7 \pm 7.8$ vs. $108.2 \pm 8.0 \mathrm{mmHg}$, $\mathrm{p}=0.02$ ). Bronchoaspiration or the PEEP change did not lead to a significant effect on these parameters; a decrease in MAP was observed only in Group P4 at 110 min (108 \pm 8 vs. $94.0 \pm 7.0 \mathrm{mmHg}, \mathrm{p}=0.031)$. 


\subsubsection{Gas exchange}

Arterial blood gas parameters are demonstrated in Table 1. No significant change was observed in arterial $\mathrm{pH}$ or $\mathrm{CO}_{2}$ throughout the study protocol in either group, except for a drop in $\mathrm{pH}$ in Group $\mathrm{P} 4$ following the change of PEEP $(\mathrm{p}=0.04)$, and an elevation in arterial $\mathrm{CO}_{2}$ in Group P10 at 110 min $(p=0.016)$. A significant decrement was detected in HQ for both groups at $20 \min (\mathrm{p}=0.048$ and $\mathrm{p}=0.020$ for Group P10 and Group P4, respectively), but no change was apparent thereafter.

\begin{tabular}{|lllllll|} 
& \multicolumn{2}{c}{ Arterial pH } & \multicolumn{2}{c}{ HQ (mmHg) } & \multicolumn{2}{c}{ Arterial $\mathbf{C O}_{2}(\mathbf{m m H g})$} \\
& Group P10 & Group P4 & Group P10 & Group P4 & Group P10 & Group P4 \\
\hline 0 min & $7.40 \pm 0.03$ & $7.43 \pm 0.02$ & $507 \pm 59$ & $475 \pm 19$ & $51.00 \pm 5.80$ & $49.90 \pm 4.67$ \\
\hline 20min & $7.34 \pm 0.04$ & $7.38 \pm 0.02 \#$ & $365 \pm 52 \#$ & $374 \pm 58 \#$ & $60.38 \pm 4.92$ & $56.06 \pm 4.77$ \\
\hline 80 min & $7.35 \pm 0.05$ & $7.35 \pm 0.02$ & $388 \pm 72$ & $472 \pm 16$ & $59.84 \pm 6.66$ & $60.60 \pm 2.97$ \\
\hline $\mathbf{1 1 0}$ min & $7.30 \pm 0.06$ & $7.36 \pm 0.02$ & $384 \pm 91$ & $494 \pm 23$ & $69.43 \pm 8.98 \#$ & $59.00 \pm 3.19$ \\
\hline
\end{tabular}

Table 1. Arterial $\mathrm{pH}$, Horowitz quotient $(\mathrm{HQ})$ and arterial partial $\mathrm{CO}_{2}$ pressure in

Groups P10 and P4 under baseline conditions (0 min) and following aspiration.

${ }^{\#}: p<0.05$ vs. baseline conditions within the same group.

\subsection{Respiratory consequences of blood loss and fluid replacement: results in rats}

The body weights did not exhibit statistically significant differences between the protocol groups $(344 \pm 16.1 \mathrm{~g}$ for Group B, $320 \pm 51.24 \mathrm{~g}$ for Group CO and $361 \pm 20.7 \mathrm{~g}$ for Group $\mathrm{CR})$. Table 2 demonstrates the baseline values of the respiratory mechanical parameters for the three experimental groups. No statistically significant differences were detected in the variables reflecting the airway or tissue mechanics.

\begin{tabular}{|lccc|} 
& Raw $\left(\mathrm{cmH}_{2} \mathrm{O} . \mathrm{s} / \mathrm{l}\right)$ & $\mathbf{G}\left(\mathrm{cmH}_{2} \mathrm{O} / \mathrm{l}\right)$ & $\mathbf{H}\left(\mathrm{cmH}_{2} \mathrm{O} / \mathrm{l}\right)$ \\
\hline Blood & $54.4(2.7)$ & $1034(33.3)$ & $5332(272)$ \\
\hline Colloid & $52.3(3.9)$ & $1061(46.9)$ & $5293(407)$ \\
\hline Crystalloid & $51.7(3.3)$ & $912(35.0)$ & $4533(182)$ \\
\hline
\end{tabular}

Table 2. Mean (SE) values of the airway resistance (Raw), tissue damping $(G)$ and elastance $(H)$ obtained under the control conditions in the three groups of rats. 


\subsubsection{Blood gas parameters}

The arterial blood gas parameters obtained at the beginning, at the midpoint and at the end of the experimental protocol are presented in Table 3. In Group B, Hct did not exhibit statistically significant changes throughout the protocol, whereas decreases in $\mathrm{pH}(\mathrm{p}<0.001)$ and $\mathrm{PaO}_{2}(\mathrm{p}=0.011)$ were evidenced. As compared with the autologous blood, fluid replacement with colloid solution resulted in a lower Hct $(p<0.001)$, while crystalloid administration led to significant reductions in Hct $(\mathrm{p}=0.010)$ and $\mathrm{pH}(\mathrm{p}=0.009)$. No difference in the changes in $\mathrm{PaO}_{2}$ and $\mathrm{pH}$ was observed between the rats in Groups $\mathrm{CR}$ and CO. The decreases in Hct were more pronounced in Group CO than those in Group CR $(p=0.032)$.

\begin{tabular}{|ccccccccc|}
\hline & \multicolumn{2}{c}{ Hct $(\%)$} & \multicolumn{2}{c}{ pH } & \multicolumn{2}{c|}{$\mathrm{PaO}_{2}(\mathrm{mmHg})$} & \multicolumn{2}{c|}{$\mathrm{PaCO}_{2}(\mathrm{mmHg})$} \\
& $\mathrm{W} 1$ & $\mathrm{~W} 6$ & $\mathrm{~W} 1$ & $\mathrm{~W} 6$ & $\mathrm{~W} 1$ & $\mathrm{~W} 6$ & $\mathrm{~W} 1$ & $\mathrm{~W} 6$ \\
\hline \multirow{2}{*}{ Blood } & 34.4 & 33.9 & 7.52 & $7.43^{*}$ & 79.4 & $63.3^{*}$ & 26.6 & 29.6 \\
& $(1.2)$ & $(0.85)$ & $(0.02)$ & $(0.01)$ & $(5.6)$ & $(5.1)$ & $(1.8)$ & $(2.1)$ \\
\hline Colloid & 32.7 & $24.2^{* \#}$ & 7.51 & $7.40^{*}$ & 80.4 & $63.7^{*}$ & 23.8 & 26.3 \\
& $(1.3)$ & $(1.8)$ & $(0.02)$ & $(0.02)$ & $(3.1)$ & $(2.4)$ & $(1.3)$ & $(2.0)$ \\
\hline Crystalloid & 33.8 & $28.5^{* \# \$}$ & 7.51 & $7.36^{* \#}$ & 79.9 & $64.9^{*}$ & 23.9 & 21.5 \\
& $(0.76)$ & $(1.9)$ & $(0.02)$ & $(0.02)$ & $(5.6)$ & $(4.1)$ & $(1.9)$ & $(4.5)$ \\
\hline
\end{tabular}

Table 3. Mean (SE) parameter values derived from arterial blood samples obtained at the first (W1) and the last (W6) withdrawal.

*: $p<0.05$ W1 vs. W6; ${ }^{\#}: p<0.05$ vs. blood; $\$: p<0.05$ vs. colloid.

\subsubsection{Respiratory mechanics}

Figure 10 depicts the changes in the airway and respiratory tissue mechanical parameters relative to the baseline. Blood withdrawal resulted in a systematic lowering of Raw. The fluid replacement with colloid in Group CO restored the baseline value of Raw, whereas the Raw remained diminished following the iv administration of autologous blood in Group B $(p=0.005)$. The changes in Raw after the iv injections of crystalloid solution in Group CR were intermediate $(\mathrm{p}<0.038)$, with less obvious elevations in Raw after the third fluid replacement manoeuvre. Monotonous increases in $\mathrm{G}$ were observed throughout the protocol $(\mathrm{p}<0.001)$, with no statistically detectable differences between the protocol groups. $\mathrm{H}$ was 
elevated in all groups, with significantly greater changes in Groups $\mathrm{CR}(\mathrm{p}=0.005)$ and $\mathrm{CO}$ $(\mathrm{p}=0.012)$ than in Group B.

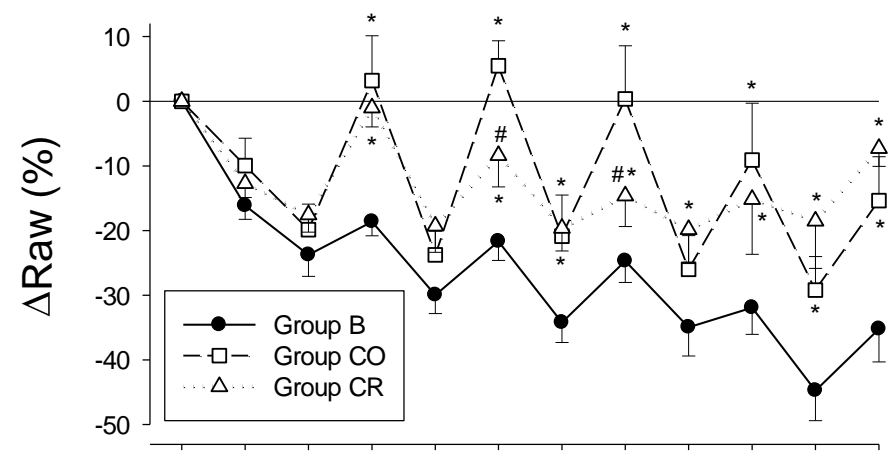

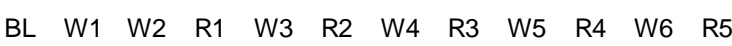
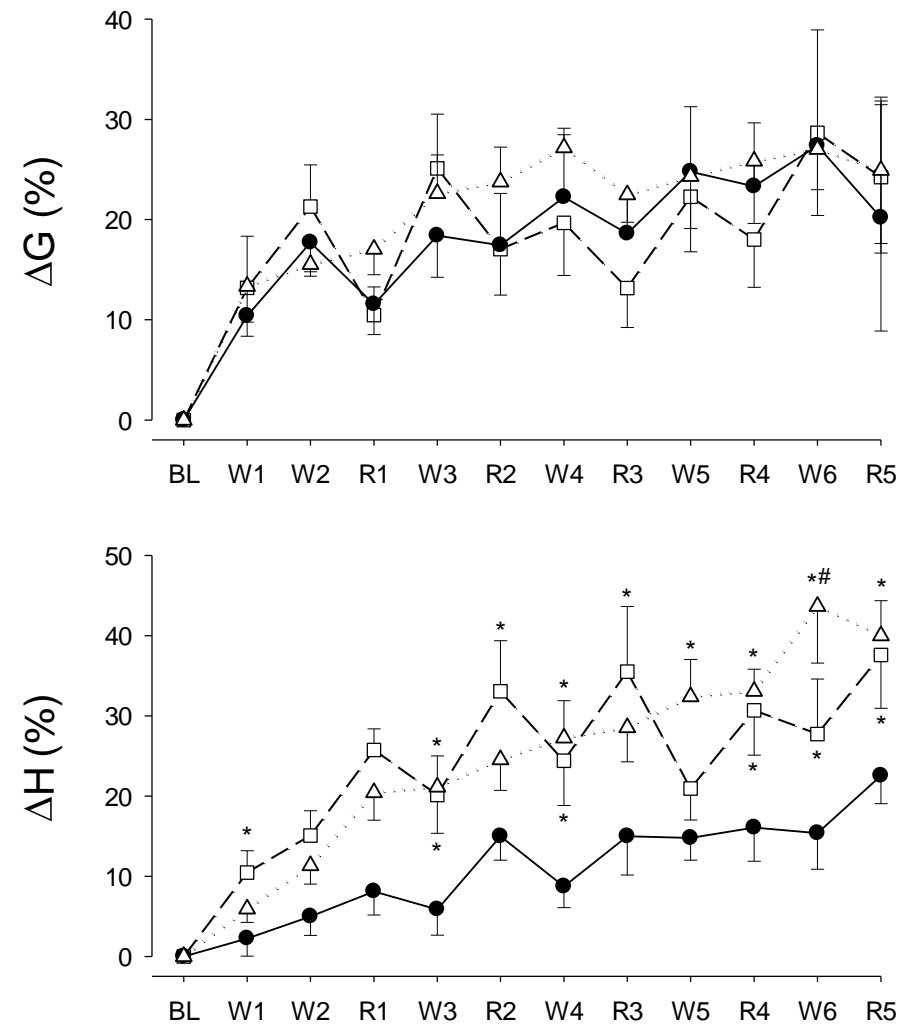

Experimental condition

Figure 10. Changes in the airway and tissue mechanics relative to the baseline.

Raw: airway resistance, G: tissue damping, $H$ : tissue elastance, BL: baseline. W1-W6: blood withdrawals, R1-R5: fluid replacements with autologous blood (Group B), colloid (Group CO) or crystalloid (Group CR). *: $p<0.05$ vs. Group B within a condition; ${ }^{\#}: p<0.05$ vs. Group $C O$ within a condition. 


\subsubsection{Haemodynamic changes}

The systemic haemodynamic changes for the 3 groups of rats are displayed in Figure 11. The blood withdrawals caused MAP to decrease systematically, while it was restored to the previous values by fluid replacements, regardless of the group allocation. HR displayed gradual increases in all groups of rats, with significant changes from R3, W3 and R2 in Groups B, CR and CO, respectively.
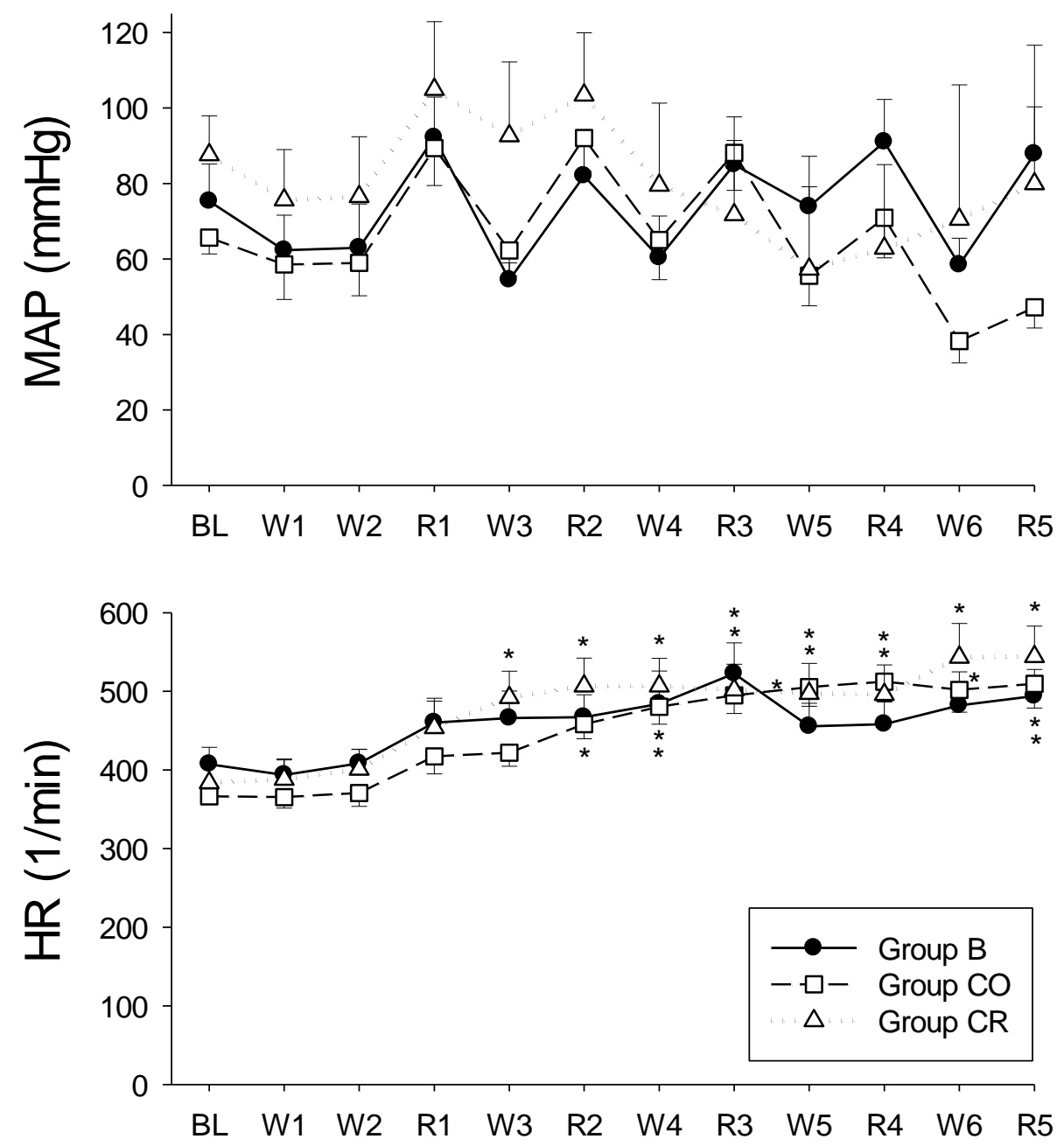

Figure 11. Systemic haemodynamic parameters.

MAP: mean arterial pressure; HR: heart rate, BL: baseline, W1-W6: blood withdrawals, R1-R5: fluid replacements with autologous blood (Group B), colloid (Group CO) or crystalloid (Group CR). *: $p<0.05$ vs. BL within a group. 


\subsubsection{Lung oedema}

The oedema parameters obtained from the lung weights and from the histological analyses are to be seen in Figure 12. The animals in both Groups CR and CO exhibited significantly greater wet-to-dry lung weight ratios ( $p<0.001$ for both), as also manifested in the perivascular pulmonary oedema indices ( $\mathrm{p}<0.05$ for both).
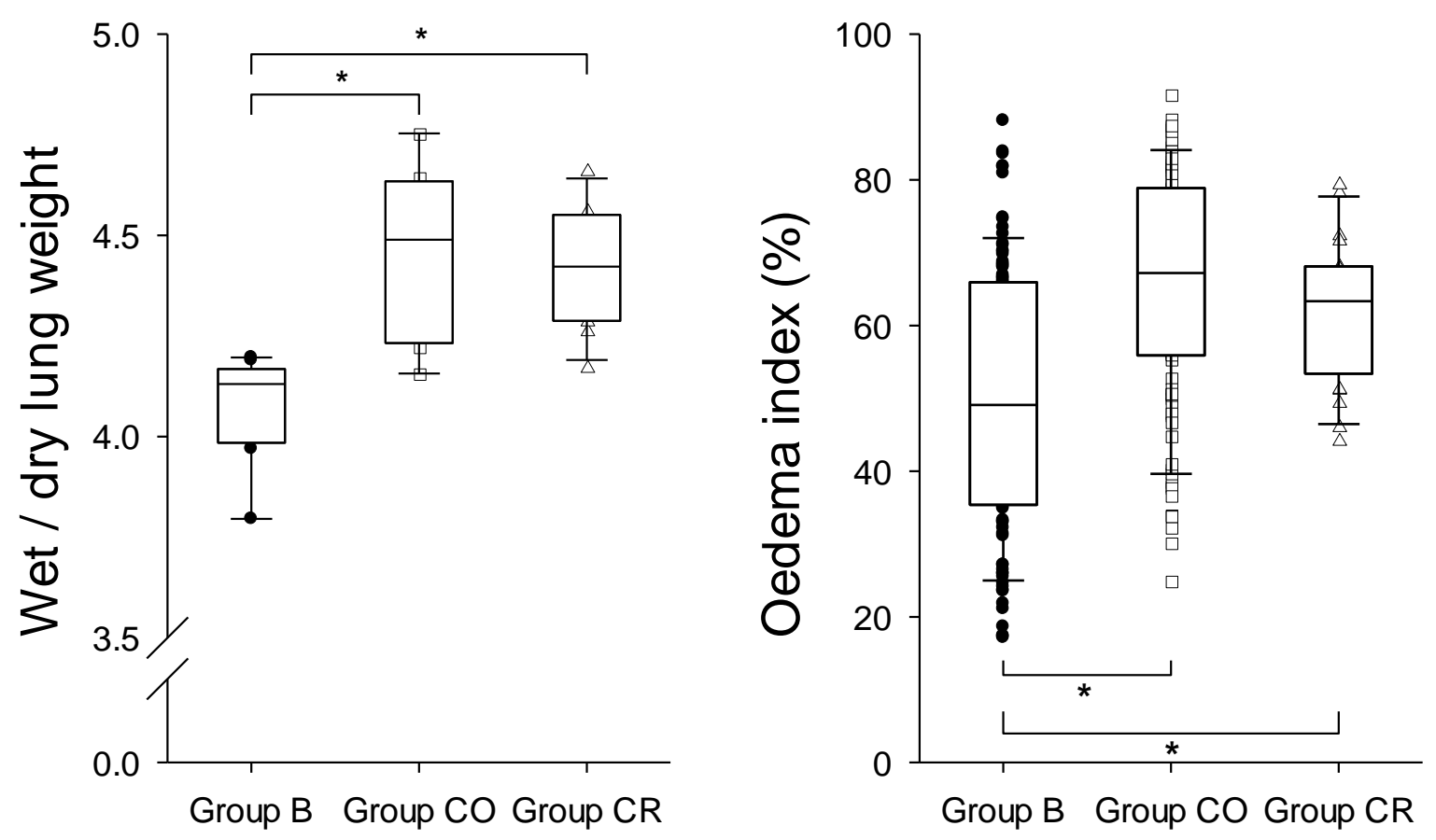

Figure 12. Oedema indices following fluid replacement by various solutions.

Obtained by relating the wet lung weight to the dry weight (left) and by relating the perivascular oedema area to the total vessel area on histological sections (right) obtained in rats receiving autologous blood (Group B), colloid (Group CO) or crystalloid (Group CR). *: $p<0.05$.

The relationships between the wet-to-dry lung weight ratio and the relative change in $\mathrm{H}$ are presented in Figure 13. Pooling of the data from the 3 protocol groups revealed significant correlations between the macroscopic oedema index and the increased stiffness of the respiratory system $(R=0.55, \mathrm{p}<0.01)$. 


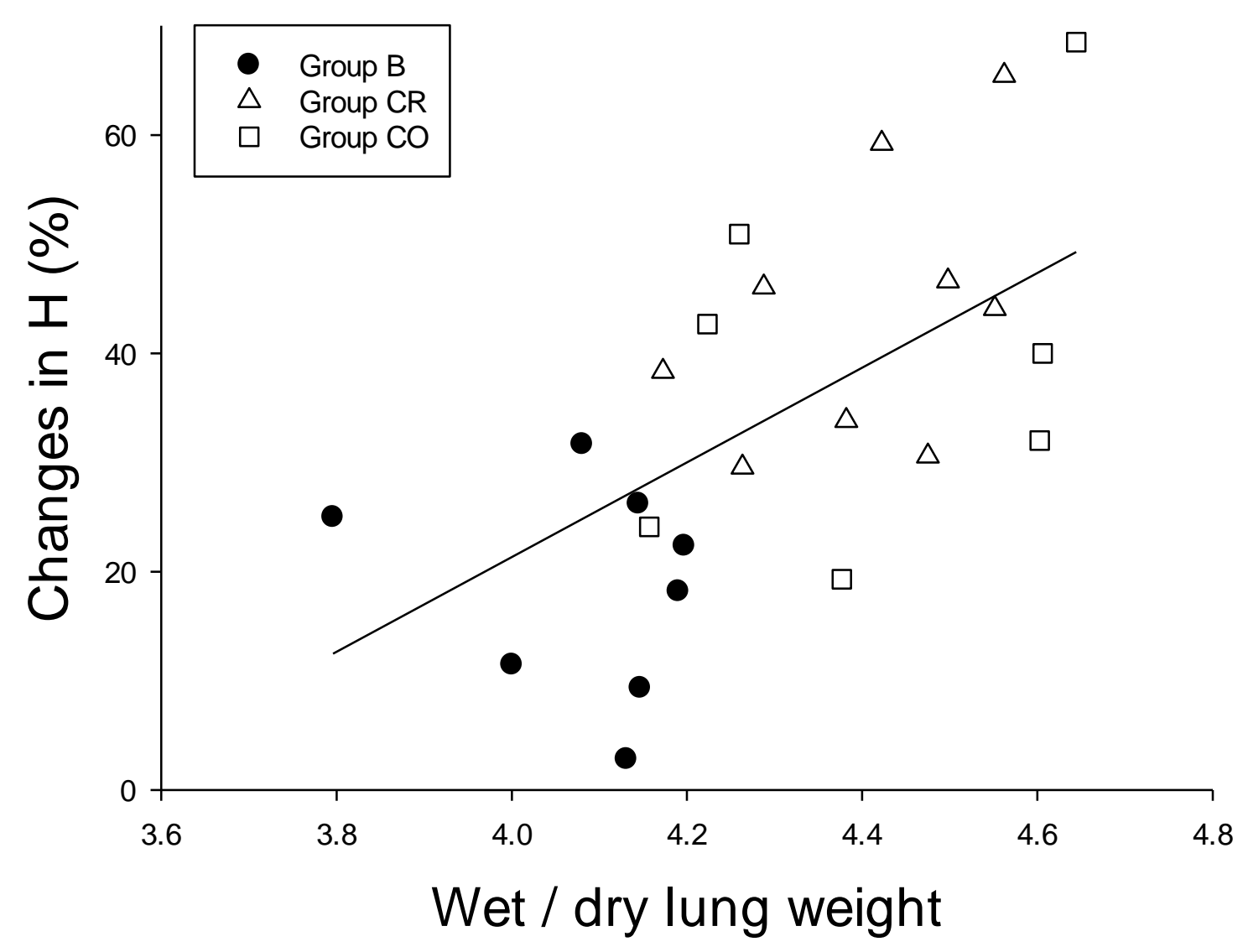

Figure 13. Relationship between the changes in oedema index and in respiratory elastance in rats.

The oedema index is represented by wet weight/dry weight, $H$ : tissue elastance. Obtained in rats receiving autologous blood (Group B), colloid (Group CO) or crystalloid (Group CR). 


\section{Discussion}

In the studies reported in the present thesis, we examined the respiratory mechanical changes following adverse events responsible for perioperative respiratory morbidities during general anaesthesia and intensive care.

The inhalation of oropharyngeal or gastric contents into the lower respiratory tract demands an immediate intervention from health-care providers in order to minimize the adverse pulmonary consequences. Our results revealed the dynamics of the changes in respiratory mechanics following unilateral bronchoaspiration by demonstrating a rapid development of adverse changes in the affected lung, with a peak deterioration in the first 15 min and gradual improvements thereafter. We further demonstrated that the adverse respiratory consequences of bronchoaspiration can be attenuated be elevating the PEEP. Separate assessment of the affected and intact lungs following bronchoaspiration evidenced the predominance of the local direct damage over the indirect systemic effects in the development of the deterioration of the lung function.

Another clinical situation involving an increased risk of respiratory morbidities is related to fluid resuscitation, since adverse lung functional changes are anticipated subsequent to the potential development of pulmonary oedema. In an animal model mimicking fluid replacement during surgical bleeding, we have acquired experimental evidence of similar short-term respiratory consequences following crystalloid or colloid replacement therapies of acute blood loss.

\subsection{Effects of bronchoaspiration on respiratory function}

In the first study of the present thesis, changes in the lung mechanical and ventilation properties were measured for the left and right lungs separately following unilateral gastric juice aspiration. This approach allowed a within-subject comparison of the local and indirect systemic effects of the bronchoaspiration by measuring the affected and the intact lung separately. Our results revealed that the marked acute lung mechanical responses observed in the airway and tissue mechanical properties in the aspirated lung were not associated with any detectable deterioration on the intact side at the 2-h follow-up after aspiration. Nevertheless, there was a significant association between the magnitude of the airway response in the intact 
lung and that in the aspirated lung. The presence of an uneven ventilation of the aspirated lungs was obvious from the values of the elevated normalized capnogram third phase slope. However, this change was not seen in the intact lung, in accordance with the forced oscillatory mechanical findings. The elevation of the PEEP under these conditions prevented the adverse changes in the capnogram third phase slope, while it had only minor effects on the other lung mechanical, ventilation or oxygenation indices.

In agreement with the findings of the few previous studies in which the mechanical changes were assessed for both lungs together in various animal models, we observed an acute deterioration in the lung tissue mechanics in the aspirated lung [28, 46, 47]. This adverse change can be attributed to pulmonary oedema due to the increased vascular permeability [28, 48, 49], surfactant dysfunction [19, 50] and inflammation [25, 26]. Since all of these processes promote the development of atelectasis leading to a lung volume loss, the elevations in the unilateral $\mathrm{G}$ and $\mathrm{H}$ in the aspirated lungs are likely to occur subsequently to this phenomenon. The scarce earlier results indicated similar detrimental changes in the total resistive properties of the pulmonary system $[46,51,52]$. However, we are unaware of any previous studies assessing changes in airway mechanics following acid aspiration. The marked acute rise in Raw indicates the development of a transient bronchial smooth muscle contraction in the aspirated lung. Although the mechanisms responsible for this finding have not been fully clarified, it may be explained by the direct effect of the epithelial damage on the airway smooth muscle contractility [53], or by the localized release of bronchoconstrictive neurotransmitters, such as tachykinin and bradykinin by capsaicin-sensitive neurons [54].

Respiratory abnormalities were also assessed in the present study by measuring $S_{\text {III }}$ and $S_{\text {nIII. }}$ The extent of emptying of lung compartments containing various $\mathrm{CO}_{2}$ concentrations during mechanical ventilation and the shape of the resulting capnogram are determined primarily by the airway geometry [55-59], and the driving pressure exerted by the elastic recoil of the respiratory tissues [60]. The marked elevations in these parameters in the aspirated lungs suggest the presence of uneven lung ventilation, particularly at the lower PEEP level (Figure 9). This may be subsequent to the localized deposition of the instilled gastric juice $[46,61]$, which compromised the regional ventilation in these patchy lung areas. 
Our findings also demonstrate the transient feature of this disturbance, since the ventilation abnormalities were no longer detectable $60 \mathrm{~min}$ after the aspiration. This temporal pattern suggests that the period $0-60 \mathrm{~min}$ is the optimum time window for the therapeutic application of an elevated PEEP against lung peripheral ventilation abnormalities following bronchoaspiration.

An elevation of the PEEP is commonly used to prevent persistent atelectasis formation following aspiration. Our results demonstrate no major benefit of this manoeuvre on either the airway or the lung tissue mechanics (Figure 7). This finding agrees with previous results where no beneficial effect of a PEEP rise on the respiratory compliance was demonstrated following acid aspiration [28]. However, the lack of an increase in $S_{\text {III }}$ in Group P10 indicates that the elevated PEEP prevented the development of ventilation defects in the lung periphery. This benefit was not associated with detrimental changes in the systemic haemodynamics or gas exchange. Indeed, compromised systemic haemodynamic changes have been reported only at higher PEEP levels, such as $15 \mathrm{cmH}_{2} \mathrm{O}$ [30]. This finding indicates that a moderately elevated PEEP has a beneficial effect on the aspiration-induced ventilation defects without affecting the systemic haemodynamics.

The most noteworthy finding of the present study is the experimental evidence that all these detrimental changes in the lung mechanics and ventilation were markedly repressed on the intact lung side, i.e. bronchoconstriction was detected only in the animals that responded strongly to unilateral aspiration (Figure 8). This finding can be interpreted as it reveals a link between direct and indirect mechanisms by demonstrating the occasional presence of bronchoconstriction in the intact lung only if the bronchospasm was severe in the affected lungs. Overall, the markedly smaller responses in the intact lungs reflect the negligible role of the indirect constrictor mechanisms of systemic origin. However, it is also possible that the lack of change in the intact lungs following aspiration may result from the opposing effects of the elevated vagal tone leading to lung constriction [20,21] and the endogenous release of catecholamines with relaxation potentials [62]. Independently of the mechanism, our data indicate the predominance of local effects over systemic effects in the aspiration-induced deterioration of lung function. 
Our observations agree with those of previous studies, where local effects prevailed in the early stage of aspiration (the first 4-6 h) [16]. Mechanisms proposed to explain this local damage include the direct corrosive effect of the aspirated gastric content [17], the localized acute airway inflammation caused by the pepsin in gastric juice [18], the localized release of bronchoactive mediators and the subsequent injury of the alveolar epithelium [61]. Earlier studies pointed to the systemic effects predominating in the later stages [63]; these are related mainly to inflammatory mechanisms, such as the activation of neutrophils [23, 64] or alveolar macrophages.

\subsubsection{Methodological aspects}

A number of methodological issues relating to the present experimental model warrant consideration. The model applied in the present study mimics the most important clinical features of bronchoaspiration, such as bronchospasm and atelectasis development leading to impaired oxygenation. However, the effects of aspiration on the lung oxygenation and on the systemic haemodynamics in the present study were less severe in magnitude than those obtained earlier [46]. A plausible explanation for this apparent discrepancy may be the unilateral aspiration applied in the current experiments. This approach left one of the lungs intact, which ensured low mortality with relatively mild systemic consequences, similarly as with previously described models where unilateral acid aspiration was applied, but without assessing the functional changes in the lung in the aspirated and intact lungs individually [61, 63].

A further methodological issue relates to the ventilation mode involved in our porcine model. To maintain the minute ventilation following aspiration, the volume-controlled mode was selected. However, this mode of mechanical ventilation may lead to a mild overinflation of the intact lungs following unilateral aspiration via a redistribution of the pre-set tidal volume [65], especially when a relatively high PEEP is applied. This phenomenon may provide a plausible explanation for the significant lowering of Raw, G and H in Group P10 after 60 min on the intact lung side (Figures 6 and 7).

Another technical aspect of our experiments is related to the measurement of pulmonary mechanical parameters in the open chest without the potential confounding effects of the chest wall mechanics. Configurational differences of the lungs in the intact and open-chest may 
have affected the values of the airway and tissue mechanical parameters [47] and haemodynamics [66]. However, this condition was stable throughout the study protocol and the mechanical parameters were obtained during small-amplitude oscillations that minimizes the mechanical stress and thus, these factors were not likely to affect our major findings. The use of a single-compartment model to estimate airway and tissue mechanical properties may have further limitation in case of development of ventilation heterogeneities [67], which is expected to occur in the affected lungs following bronchoaspiration. In this case, the increases in $G$ may overestimate the real changes in the parenchymal viscoelasticity due to the involvement of an artefactual component related to enhanced ventilation inhomogeneities.

\subsubsection{Summary}

In summary, we have developed an animal model with which to distinguish the local and the systemic effects of bronchoaspiration. Our study has provided experimental evidence that unilateral aspiration of the gastric contents leads to a unilateral lung injury in the first $2 \mathrm{~h}$, characterized by acute elevations of the airway and tissue mechanical and capnogram third phase slope parameters, but exclusively on the affected side. This indicates the primary need for treatment of the local detrimental consequences of bronchoaspiration in the acute phase (the first $2 \mathrm{~h}$ ) rather than targeting the adverse systemic changes. Application of a moderately high PEEP prevented the adverse changes in the third phase slope of the capnogram, indicating decreased ventilation heterogeneities. This benefit was not associated with changes in other mechanical, cardiovascular or lung oxygenation indices, suggesting that a moderately high PEEP may be beneficial in the optimum lung management following bronchoaspiration.

\subsection{Respiratory effects of blood loss and fluid replacement}

In the second study of the present thesis, we applied an experimental model that mimics continuous insidious surgical bleeding and fluid replacement for a direct assessment of the mechanical properties specific for the airway and respiratory tissues following a blood loss and its treatment with solutions commonly used in clinical practice. The decreased airway resistance subsequent to the haemorrhage remained low after fluid therapy with autologous blood, whereas it has re-elevated back to baseline by the administration of colloid and increased partially by fluid replacement with crystalloid. The respiratory tissues stiffened more markedly in the animals receiving colloid or crystalloid, with no difference in effect 
between these solutions. These adverse tissue mechanical changes were also reflected in the alterations in the oedema indices determined by lung weighing and by histology.

There has recently been an extensive debate concerning the optimum fluid replacement therapy following blood loss from the aspects of the type and the amount of the administered solution. While the lungs are primarily affected in consequence of the fluid therapy, no information is currently available on the airway and tissue mechanical changes. The administration of blood products is often considered to ensure the oxygen transport by maintaining the normal haemoglobin content. Restoration of the circulatory blood volume by blood products has a beneficial effect on the preservation of sufficient microcirculation with minimal morphologic damage or ischaemic cell injury [68, 69]. Similarly to previous findings, the blood volume loss in the present study led to bronchodilation, which is most probably due to the compensatory increase in thoracic gas volume and/or the elevated levels of circulatory catecholamines [38]. The present findings extend these results in a different model of haemorrhage without inducing the severe hypovolaemia characteristic of hidden, leaking bleeding during major surgery.

While recent studies have focused on the morbidity and mortality related to colloid or crystalloid administrations as fluid replacement therapy $[31,32,70]$, the pulmonary effects of these solutions are mainly based on empirical investigations without firm evidence [35-37]. As far as we are aware, the present study addresses for the first time the respiratory mechanical changes in response to common fluid replacement strategies and attempts to establish their relationship to the oedema indices in an experimental model of surgical bleeding.

Our results demonstrate that the Raw essentially remains lowered after administration of autologous blood. The lack of a complete recovery in airway tone may be related to the relaxation potential of heparin [71]. However, a comparison of heparinized and nonheparinized colloid solutions revealed no difference in their bronchial effects (data not shown), and the potential role of heparin can therefore be excluded. Alternatively, the depressed Raw may be attributed to the presence of bronchoactive mediators in the sequestered blood, with the particular importance of the increased levels of adrenaline and noradrenaline in the withdrawn and subsequently re-administered blood [38]. Conversely, the 
findings revealed a complete reversal of the haemorrhage-induced bronchodilation by colloid. This suggests the importance of the interactions between circulatory changes and airway mechanics following a blood loss, with recovery of the original airway geometry through restoration by approaching the initial circulatory volume. The increases in Raw following colloid administration may be attributed to a distension of the bronchial submucosal vessels and/or to the oedema formation resulting in airway wall thickening, or an exudation into the airway lumen [72]. A similar concept can be applied to the initial results obtained with crystalloid solution, the first administration of which fully reversed the decreased Raw, when its entire volume was likely to remain in the vascular bed. This effect of the elevated intravascular volume may have been abolished in the rats of Group B due to the presence of catecholamines in the readministered autologous blood.

The tissue viscoelastic parameters following blood administration revealed slight, gradual increases, which can be attributed to the atelectasis and subsequent lung volume loss induced by the anaesthesia and mechanical ventilation in the supine position. This phenomenon was confirmed by the decrease in $\mathrm{PaO}_{2}$, which suggests the loss of alveolar surface available for gas exchange. An important finding of the present study is the more marked gradual impairment of the respiratory tissue viscoelasticity in the animals receiving colloid or crystalloid solution (Figure 10). This difference may arise from the variable rheological properties of the administered fluids that may contribute to the altered respiratory tissue behaviour [73], or from haemodilution-related changes in the colloid osmotic pressures. These phenomena result in oedema development affecting directly the tissue viscoelasticity. Since these adverse changes were also reflected in the oedema indices (Figures 12 and 13), the primary role of the accumulating perivascular oedema fluid in the compromised respiratory tissue stiffness can be anticipated. It is noteworthy that no evidence of a difference was found between colloid and crystalloid treatments either in the changes in the tissue mechanics or in the oedema indices. These results suggest the equivalence of these fluid replacement strategies in terms of compromising the lung tissue viscoelasticity, and as regards pulmonary oedema formation. This correspondence is also reflected in the lack of difference in the changes of blood oxygenation following the two fluid replacement regimes (Table 3). These findings are in qualitative agreement with previous results that demonstrated the lack of difference between colloid and crystalloid solutions in influencing the extravascular lung 
water, pulmonary leak index and lung injury score [74-76]. However, it should be borne in mind that this may hold true only in the relatively short time frame ( $90 \mathrm{~min})$ covered by our protocol, and systematic assessments of the prolonged effects would require further investigations.

\subsubsection{Methodological aspects}

An important methodological aspect of our protocol is related to the nature and the volume of the administered fluid. There is still a debate in the literature on the nature of fluid to be considered for resuscitation [77]. Conflicting reports aroused from recent meta-analyses with some authors suggesting the safe use of albumin in critically ill patients [78], while others do not recommend its use because of lack of robust evidence for its effectiveness to reduce mortality [79]. The very recent international consensus promotes the use of crystalloids against both HES and albumin solutions [77]. Since the aim of the present study was primarily to compare the effect of three basic fluid replacement strategies, we deliberately selected HES, as the colloid solution comparator. As concerns the crystalloid solution, various types are available for fluid replacement therapy, with slightly different ingredients resulting in somewhat variable osmolarities. Since there is an evolving debate on the choice of balanced salt solutions and normal saline, this latter offers similar osmolarity with the other 2 fluid replacement strategies. Thus, isotonic normal saline rather than the hypotonic Ringer's lactate was selected for a comparison with the slightly hypertonic HES $6 \%$ 130/0.4 [80]. As concerns the volume of crystalloid solution for fluid replacement, no evidence-based recommendations are available. Whereas textbooks conventionally state that the volume of crystalloids to be administered should be 3- to 4-fold the blood loss [81], recent studies question this, suggesting a ratio close to $1: 1$ [32, 37, 82]. Since the acute effect of fluid replacement was at the focus of interest in the present study, the same volume was chosen for the blood loss and replacement for both solutions (5\% of the total blood volume). The rationale of this approach was confirmed by the lack of difference in MAP and HR between the protocol groups, which agrees with the concept of goal-directed therapy [82, 83].

A methodological limitation of our findings is associated with the use of total respiratory impedance data to assess pulmonary changes. While Raw accurately reflects the flow resistance of the airways, the chest wall contributes significantly to the tissue parameters $G$ 
and $\mathrm{H}$ [44]. Nevertheless, the viscoelastic properties of the chest wall exhibited negligible changes following the induction of severe oedema with oleic acid [84]. Thus, our results are likely to reflect pulmonary changes; however, their magnitude may be somewhat underestimated due to the masking effect of the chest wall. Another methodological limitation is related to the species difference between small rodents and humans necessitating careful extrapolation of our data to a clinical situation. While rats have substantially higher Raw, G and $\mathrm{H}$ than humans, no major differences exist between mammalian species in the oscillatory mechanics besides scaling factor [85].

\subsubsection{Summary}

In summary, our results have provided experimental evidence of the dissociated changes in the airway and tissue mechanical properties following surgical-type bleeding and its treatment with autologous whole blood, colloid or crystalloid solution in a volume that fully restored mean arterial pressure. The measurement of respiratory mechanical, histological and gas exchange consequences of blood loss and consecutive fluid replacement strategies revealed no differences between fluid replacement with colloid and crystalloid. The two solutions demonstrated similar abilities to compromise the lung tissue viscoelasticity subsequent to mild perivascular oedema formation. These findings highlight the differences in behaviour of the respiratory system following fluid replacement with blood, colloid or crystalloid: a sustained bronchodilation is expected after the administration of autologous blood, without significant lung tissue changes, whereas colloids and crystalloids tend to restore the basal airway tone at the expense of similar deteriorations in lung tissue viscoelasticity. 


\section{Conclusions}

Aspiration of the gastric contents, blood loss and fluid replacement are important factors of perioperative morbidity. Respiratory effects are among the most critical changes related to their morbidity.

In the present thesis, an animal model was established for the separate investigation of the mechanical properties of the left and the right lung. The use of a double-lumen ET tube allowed the independent introduction of the forcing signal to each lung, allowing characterization of the changes in the mechanical properties of each lung. We have demonstrated that the initial changes induced by bronchoaspiration are dominated by the direct effects, since the unaffected lung was not altered in terms of lung mechanics, and that the application of moderate PEEP is beneficial in the management of bronchoaspiration.

We have also adapted a well-established animal model for the investigation of acute blood loss, but also improved it for the examination of the effects of various methods of fluid replacement. The sequential removal and replacement of blood mimicked continuous, but hidden surgical bleeding with its replacement. Our measurements did not reveal a difference between fluid replacement with colloid or crystalloid, represented in comparable oedema formation and adverse changes in the viscoelastic properties of the lung.

These findings contribute to a better understanding of the underlying mechanisms of these anaesthesia-related complications through a description of the not yet characterized respiratory changes initiated by bronchoaspiration of the gastric contents and by acute blood loss and its fluid replacement. If it is assumed that the same mechanisms are present in humans, this better understanding might further improve the management of these adverse perioperative events and reduce their morbidities. 


\section{Acknowledgements}

First of all, I would like to express my gratitude to my supervisor, Ferenc Peták, for inviting me to his research group as a student in 2009 and for helping me through the difficulties of science ever since. I greatly appreciate his mentoring and trust in me.

I would like to thank Barna Babik for his invaluable advice and for his support during my research career. He has greatly influenced me.

I am grateful to Walid Habre for his support and the numerous opportunities he gave me to perform research in his laboratory.

I would like to thank Professor Ferenc Bari for enabling me to carry out my research in the Department of Medical Physics and Informatics and for his trust in me.

I would like to thank the co-authors of my papers, for helping me in preparing them. I am grateful to the staff of the Institute of Surgical Research at the University of Szeged for their cooperation in the pig study, and to Orsolya Ivánkovitsné Kiss for her invaluable technical help in the rat study. I would like to thank Ádám Balogh for his help in both studies and his advice regarding this thesis. I would like to thank all my colleagues in the Department of Medical Physics and Medical Informatics for creating a friendly environment.

I am grateful to Judit and my family for their constant support.

The studies included in this thesis were supported by a Hungarian Scientific Research Grant (OTKA K81179) and the European Union and the State of Hungary, co-financed by the European Social Fund in the framework of TÁMOP 4.2.4.A/2-11-1-2012-0001 'National Excellence Program' and 4.2.2.A-11/1/KONV-2012-0052. 


\section{References}

1. Weiser TG, Regenbogen SE, Thompson KD, Haynes AB, Lipsitz SR, Berry WR, Gawande AA: An estimation of the global volume of surgery: a modelling strategy based on available data. Lancet 2008, 372:139-144.

2. Smetana GW: Preoperative pulmonary evaluation. $N$ Engl J Med 1999, 340:937944.

3. Fisher BW, Majumdar SR, McAlister FA: Predicting pulmonary complications after nonthoracic surgery: a systematic review of blinded studies. Am J Med 2002, 112:219-225.

4. Mazo V, Sabate S, Canet J, Gallart L, de Abreu MG, Belda J, Langeron O, Hoeft A, Pelosi P: Prospective external validation of a predictive score for postoperative pulmonary complications. Anesthesiology 2014, 121:219-231.

5. Standards for Basic Anesthetic Monitoring. Retrieved on 14 January 2016 from [http://www.asahq.org/ /media/Sites/ASAHQ/Files/Public/Resources/standardsguidelines/standards-for-basic-anesthetic-monitoring.pdf]

6. Babik B, Petak F, Asztalos T, Deak ZI, Bogats G, Hantos Z: Components of respiratory resistance monitored in mechanically ventilated patients. Eur Respir J 2002, 20:1538-1544.

7. Marik PE: Aspiration pneumonitis and aspiration pneumonia. N Engl J Med 2001, 344:665-671.

8. Marik PE: Pulmonary aspiration syndromes. Curr Opin Pulm Med 2011, 17:148154.

9. Olsson GL, Hallen B, Hambraeus-Jonzon K: Aspiration during anaesthesia: a computer-aided study of $\mathbf{1 8 5 , 3 5 8}$ anaesthetics. Acta Anaesthesiol Scand 1986, 30:84-92.

10. LeFrock JL, Clark TS, Davies B, Klainer AS: Aspiration pneumonia: a ten-year review. Am Surg 1979, 45:305-313.

11. Cameron JL, Mitchell WH, Zuidema GD: Aspiration pneumonia. Clinical outcome following documented aspiration. Arch Surg 1973, 106:49-52. 
12. Warner MA, Warner ME, Weber JG: Clinical significance of pulmonary aspiration during the perioperative period. Anesthesiology 1993, 78:56-62.

13. Engelhardt $\mathrm{T}$, Webster NR: Pulmonary aspiration of gastric contents in anaesthesia. Br J Anaesth 1999, 83:453-460.

14. Mendelson CL: The aspiration of stomach contents into the lungs during obstetric anesthesia. Am J Obstet Gynecol 1946, 52:191-205.

15. Raghavendran K, Nemzek J, Napolitano LM, Knight PR: Aspiration-induced lung injury. Crit Care Med 2011, 39:818-826.

16. Kennedy TP, Johnson KJ, Kunkel RG, Ward PA, Knight PR, Finch JS: Acute acid aspiration lung injury in the rat: biphasic pathogenesis. Anesth Analg 1989, 69:8792.

17. Wynne JW, Ramphal R, Hood CI: Tracheal mucosal damage after aspiration. A scanning electron Microscope study. Am Rev Respir Dis 1981, 124:728-732.

18. Bathoorn E, Daly P, Gaiser B, Sternad K, Poland C, Macnee W, Drost EM: Cytotoxicity and induction of inflammation by pepsin in Acid in bronchial epithelial cells. Int J Inflam 2011, 2011:569416.

19. Lamonica G, Amigoni M, Vedovelli L, Zambelli V, Scanziani M, Bellani G, Grassi A, Simonato M, Carnielli VP, Cogo PE: Pulmonary surfactant synthesis after unilateral lung injury in mice. J Appl Physiol (1985) 2014, 116:210-215.

20. Kollarik M, Ru F, Undem BJ: Acid-sensitive vagal sensory pathways and cough. Pulm Pharmacol Ther 2007, 20:402-411.

21. Nemzek JA, Kim J: Pulmonary inflammation and airway hyperresponsiveness in a mouse model of asthma complicated by acid aspiration. Comp Med 2009, 59:321-330.

22. Martling CR, Lundberg JM: Capsaicin sensitive afferents contribute to acute airway edema following tracheal instillation of hydrochloric acid or gastric juice in the rat. Anesthesiology 1988, 68:350-356.

23. Weiser MR, Pechet TT, Williams JP, Ma M, Frenette PS, Moore FD, Kobzik L, Hines RO, Wagner DD, Carroll MC, Hechtman HB: Experimental murine acid aspiration injury is mediated by neutrophils and the alternative complement pathway. $J$ Appl Physiol 1997, 83:1090-1095. 
24. Kubitz JC, Annecke T, Kemming GI, Forkl S, Kronas N, Goetz AE, Reuter DA: The influence of positive end-expiratory pressure on stroke volume variation and central blood volume during open and closed chest conditions. Eur J Cardiothorac Surg 2006, 30:90-95.

25. Nader ND, McQuiller PS, Raghavendran K, Spengler RN, Knight PR: The role of alveolar macrophages in the pathogenesis of aspiration pneumonitis. Immunol Invest 2007, 36:457-471.

26. Knight PR, Druskovich G, Tait AR, Johnson KJ: The role of neutrophils, oxidants, and proteases in the pathogenesis of acid pulmonary injury. Anesthesiology 1992, 77:772-778.

27. Dellaca RL, Santus P, Aliverti A, Stevenson N, Centanni S, Macklem PT, Pedotti A, Calverley PM: Detection of expiratory flow limitation in COPD using the forced oscillation technique. Eur Respir J 2004, 23:232-240.

28. Ko SC, Zhang H, Haitsma JJ, Cheng KC, Li CF, Slutsky AS: Effects of PEEP levels following repeated recruitment maneuvers on ventilator-induced lung injury. Acta Anaesthesiol Scand 2008, 52:514-521.

29. Colmenero-Ruiz M, Fernandez-Mondejar E, Fernandez-Sacristan MA, RiveraFernandez R, Vazquez-Mata G: PEEP and low tidal volume ventilation reduce lung water in porcine pulmonary edema. Am J Respir Crit Care Med 1997, 155:964-970.

30. Marumo CK, Otsuki DA, Fantoni DT, Margarido CB, Ambrosio AM, Pelosi P, Auler JO, Jr.: Hemodynamic effects of PEEP in a porcine model of $\mathrm{HCl}$-induced mild acute lung injury. Acta Anaesthesiol Scand 2009, 53:190-202.

31. Perel P, Roberts I, Ker K: Colloids versus crystalloids for fluid resuscitation in critically ill patients. Cochrane Database Syst Rev 2013, 2:CD000567.

32. Myburgh JA, Finfer S, Bellomo R, Billot L, Cass A, Gattas D, Glass P, Lipman J, Liu $\mathrm{B}$, McArthur $\mathrm{C}$, et al: Hydroxyethyl starch or saline for fluid resuscitation in intensive care. $N$ Engl J Med 2012, 367:1901-1911.

33. Perner A, Haase N, Guttormsen AB, Tenhunen J, Klemenzson G, Aneman A, Madsen KR, Moller MH, Elkjaer JM, Poulsen LM, et al: Hydroxyethyl starch 130/0.42 versus Ringer's acetate in severe sepsis. N Engl J Med 2012, 367:124-134. 
34. de Jonge E, Levi M: Effects of different plasma substitutes on blood coagulation: a comparative review. Crit Care Med 2001, 29:1261-1267.

35. McIlroy DR, Kharasch ED: Acute intravascular volume expansion with rapidly administered crystalloid or colloid in the setting of moderate hypovolemia. Anesth Analg 2003, 96:1572-1577, table of contents.

36. Hahn RG, Drobin D, Stahle L: Volume kinetics of Ringer's solution in female volunteers. Br J Anaesth 1997, 78:144-148.

37. Gondos T, Marjanek Z, Ulakcsai Z, Szabo Z, Bogar L, Karolyi M, Gartner B, Kiss K, Havas A, Futo J: Short-term effectiveness of different volume replacement therapies in postoperative hypovolaemic patients. Eur J Anaesthesiol 2010, 27:794-800.

38. Bayat S, Albu G, Layachi S, Portier F, Fathi M, Petak F, Habre W: Acute hemorrhagic shock decreases airway resistance in anesthetized rat. $J$ Appl Physiol 2011, 111:458-464.

39. Petak F, Habre W, Babik B, Tolnai J, Hantos Z: Crackle-sound recording to monitor airway closure and recruitment in ventilated pigs. Eur Respir J 2006, 27:808-816.

40. Petak F, Hantos Z, Adamicza A, Asztalos T, Sly PD: Methacholine-induced bronchoconstriction in rats: effects of intravenous vs. aerosol delivery. $J$ Appl Physiol 1997, 82:1479-1487.

41. Franken H, Clement J, Cauberghs M, Van de Woestijne KP: Oscillating flow of a viscous compressible fluid through a rigid tube: a theoretical model. IEEE Trans Biomed Eng 1981, 28:416-420.

42. Hantos Z, Daroczy B, Suki B, Nagy S, Fredberg JJ: Input impedance and peripheral inhomogeneity of dog lungs. $J$ Appl Physiol 1992, 72:168-178.

43. Hantos Z JT, Collins RA, Thamrin C, Sly PD: Modelling of respiratory impedance at low-to-medium frequencies (Abstract). Eur Respir J 2004, 24.

44. Petak F, Hall GL, Sly PD: Repeated measurements of airway and parenchymal mechanics in rats by using low-frequency oscillations. J Appl Physiol 1998, 84:1680-1686.

45. Lee HB, Blaufox MD: Blood volume in the rat. $J$ Nucl Med 1985, 26:72-76. 
46. Meers CM, De Wever W, Verbeken E, Mertens V, Wauters S, De Vleeschauwer SI, Vos R, Vanaudenaerde BM, Verleden GM, Van Raemdonck DE: A porcine model of acute lung injury by instillation of gastric fluid. J Surg Res 2011, 166:e195-204.

47. Petak F, Hall GL, Sly PD: Repeated measurements of airway and parenchymal mechanics in rats by using low-frequency oscillations. J Appl Physiol (1985) 1998, 84:1680-1686.

48. Grimbert FA, Parker JC, Taylor AE: Increased pulmonary vascular permeability following acid aspiration. J Appl Physiol 1981, 51:335-345.

49. Goldman G, Welbourn R, Klausner JM, Kobzik L, Valeri CR, Shepro D, Hechtman HB: Leukocytes mediate acid aspiration-induced multiorgan edema. Surgery 1993, 114:13-20.

50. Lutchen KR, Giurdanella CA, Jackson AC: Inability to separate airway from tissue properties by use of human respiratory input impedance. J Appl Physiol 1990, 68:2403-2412.

51. Kuckelt W, Dauberschmidt R, Bender V, Hieronymi U, Mrochen H, Winsel K, Althaus P, Meyer M: Gas exchange, pulmonary mechanics and haemodynamics in adult respiratory distress syndrome: experimental results in Lewe miniature pigs. Resuscitation 1979, 7:13-33.

52. Tuchman DN, Boyle JT, Pack AI, Scwartz J, Kokonos M, Spitzer AR, Cohen S: Comparison of airway responses following tracheal or esophageal acidification in the cat. Gastroenterology 1984, 87:872-881.

53. Gallos G, Townsend E, Yim P, Virag L, Zhang Y, Xu D, Bacchetta M, Emala CW: Airway epithelium is a predominant source of endogenous airway GABA and contributes to relaxation of airway smooth muscle tone. Am J Physiol Lung Cell Mol Physiol 2013, 304:L191-197.

54. Ricciardolo FL, Rado V, Fabbri LM, Sterk PJ, Di Maria GU, Geppetti P: Bronchoconstriction induced by citric acid inhalation in guinea pigs: role of tachykinins, bradykinin, and nitric oxide. Am J Respir Crit Care Med 1999, 159:557-562.

55. Yaron M, Padyk P, Hutsinpiller M, Cairns CB: Utility of the expiratory capnogram in the assessment of bronchospasm. Ann Emerg Med 1996, 28:403-407. 
56. Stromberg NO, Gustafsson PM: Ventilation inhomogeneity assessed by nitrogen washout and ventilation-perfusion mismatch by capnography in stable and induced airway obstruction. Pediatr Pulmonol 2000, 29:94-102.

57. Nik Hisamuddin NA, Rashidi A, Chew KS, Kamaruddin J, Idzwan Z, Teo AH: Correlations between capnographic waveforms and peak flow meter measurement in emergency department management of asthma. Int $J$ Emerg Med 2009, 2:83-89.

58. Bohm SH, Maisch S, von Sandersleben A, Thamm O, Passoni I, Martinez Arca J, Tusman G: The effects of lung recruitment on the Phase III slope of volumetric capnography in morbidly obese patients. Anesth Analg 2009, 109:151-159.

59. Blanch L, Lucangelo U, Lopez-Aguilar J, Fernandez R, Romero PV: Volumetric capnography in patients with acute lung injury: effects of positive end-expiratory pressure. Eur Respir J 1999, 13:1048-1054.

60. Babik B, Csorba Z, Czovek D, Mayr PN, Bogats G, Petak F: Effects of respiratory mechanics on the capnogram phases: importance of dynamic compliance of the respiratory system. Crit Care 2012, 16:R177.

61. Amigoni M, Bellani G, Scanziani M, Masson S, Bertoli E, Radaelli E, Patroniti N, Di Lelio A, Pesenti A, Latini R: Lung injury and recovery in a murine model of unilateral acid aspiration: functional, biochemical, and morphologic characterization. Anesthesiology 2008, 108:1037-1046.

62. Coupe MO, Guly U, Brown E, Barnes PJ: Nebulised adrenaline in acute severe asthma: comparison with salbutamol. Eur J Respir Dis 1987, 71:227-232.

63. Schreiber T, Hueter L, Gaser E, Schmidt B, Schwarzkopf K, Rek H, Karzai W: PEEP has beneficial effects on inflammation in the injured and no deleterious effects on the noninjured lung after unilateral lung acid instillation. Intensive Care Med 2006, 32:740-749.

64. Motosugi H, Quinlan WM, Bree M, Doerschuk CM: Role of CD11b in focal acidinduced pneumonia and contralateral lung injury in rats. Am J Respir Crit Care Med 1998, 157:192-198. 
65. Amigoni M, Bellani G, Zambelli V, Scanziani M, Farina F, Fagnani L, Latini R, Fumagalli R, Pesenti A: Unilateral acid aspiration augments the effects of ventilator lung injury in the contralateral lung. Anesthesiology 2013, 119:642-651.

66. Hoit $\mathrm{BD}$, Ball $\mathrm{N}$, Walsh $\mathrm{RA}$ : Invasive hemodynamics and force-frequency relationships in open- versus closed-chest mice. Am J Physiol 1997, 273:H25282533.

67. Lutchen KR, Hantos Z, Petak F, Adamicza A, Suki B: Airway inhomogeneities contribute to apparent lung tissue mechanics during constriction. $J$ Appl Physiol 1996, 80:1841-1849.

68. Onen A, Cigdem MK, Deveci E, Kaya S, Turhanoglu S, Yaldiz M: Effects of whole blood, crystalloid, and colloid resuscitation of hemorrhagic shock on renal damage in rats: an ultrastructural study. J Pediatr Surg 2003, 38:1642-1649.

69. Appel PL, Shoemaker WC: Evaluation of fluid therapy in adult respiratory failure. Crit Care Med 1981, 9:862-869.

70. Mutter TC, Ruth CA, Dart AB: Hydroxyethyl starch (HES) versus other fluid therapies: effects on kidney function. Cochrane Database Syst Rev 2013, 7:CD007594.

71. Abraham WM, Abraham MK, Ahmed T: Protective effect of heparin on immunologically induced tracheal smooth muscle contraction in vitro. Int Arch Allergy Immunol 1996, 110:79-84.

72. Widdicombe J: Physiologic control. Anatomy and physiology of the airway circulation. Am Rev Respir Dis 1992, 146:S3-7.

73. Mogensen ML, Steimle KS, Karbing DS, Andreassen S: A model of perfusion of the healthy human lung. Comput Methods Programs Biomed 2011, 101:156-165.

74. van der Heijden M, Verheij J, van Nieuw Amerongen GP, Groeneveld AB: Crystalloid or colloid fluid loading and pulmonary permeability, edema, and injury in septic and nonseptic critically ill patients with hypovolemia. Crit Care Med 2009, 37:1275-1281.

75. Pearl RG, Halperin BD, Mihm FG, Rosenthal MH: Pulmonary effects of crystalloid and colloid resuscitation from hemorrhagic shock in the presence of oleic acidinduced pulmonary capillary injury in the dog. Anesthesiology 1988, 68:12-20. 
76. Hahn RG: Why are crystalloid and colloid fluid requirements similar during surgery and intensive care? Eur J Anaesthesiol 2013, 30:515-518.

77. Raghunathan K, Murray PT, Beattie WS, Lobo DN, Myburgh J, Sladen R, Kellum JA, Mythen MG, Shaw AD, Group AXI: Choice of fluid in acute illness: what should be given? An international consensus. Br J Anaesth 2014, 113:772-783.

78. Xu JY, Chen QH, Xie JF, Pan C, Liu SQ, Huang LW, Yang CS, Liu L, Huang YZ, Guo FM, et al: Comparison of the effects of albumin and crystalloid on mortality in adult patients with severe sepsis and septic shock: a meta-analysis of randomized clinical trials. Crit Care 2014, 18:702.

79. Patel A, Laffan MA, Waheed U, Brett SJ: Randomised trials of human albumin for adults with sepsis: systematic review and meta-analysis with trial sequential analysis of all-cause mortality. BMJ 2014, 349:g4561.

80. Ertmer $\mathrm{C}$, Van Aken H: Fluid therapy in patients with brain injury: what does physiology tell us? Crit Care 2014, 18:119.

81. Puyana JC: Resuscitation of hypovolemic shock. In Textbook of critical care. 5th edition. Edited by Fink MP. Philadelphia: Elsevier Saunders; 2005: 1939-1940

82. Hiltebrand LB, Kimberger O, Arnberger M, Brandt S, Kurz A, Sigurdsson GH: Crystalloids versus colloids for goal-directed fluid therapy in major surgery. Crit Care 2009, 13:R40.

83. Doherty M, Buggy DJ: Intraoperative fluids: how much is too much? Br J Anaesth 2012, 109:69-79.

84. Barnas GM, Stamenovic D, Lutchen KR: Lung and chest wall impedances in the dog in normal range of breathing: effects of pulmonary edema. $J$ Appl Physiol 1992, 73:1040-1046.

85. Bates $\mathrm{JH}$, Irvin $\mathrm{CG}$, Farre $\mathrm{R}$, Hantos $\mathrm{Z}$ : Oscillation mechanics of the respiratory system. Compr Physiol 2011, 1:1233-1272. 


\section{I.}

Fodor G.H., Peták F., Érces D., Balogh Á. L., Babik B.: Lung mechanical changes following bronchoaspiration in a porcine model: differentiation of direct and indirect mechanisms. Respir Physiol Neurobiol. 2014;199:41-49. 


\title{
Lung mechanical changes following bronchoaspiration in a porcine model: Differentiation of direct and indirect mechanisms
}

\author{
Gergely H. Fodor ${ }^{\mathrm{a}}$, Ferenc Peták ${ }^{\mathrm{a}, *}$, Dániel Érces ${ }^{\mathrm{b}}$, Ádám L. Balogh ${ }^{\mathrm{c}}$, Barna Babik ${ }^{\mathrm{c}}$ \\ a Department of Medical Physics and Informatics, University of Szeged, Szeged, Hungary \\ b Institute of Surgical Research, University of Szeged, Szeged, Hungary \\ c Department of Anaesthesiology and Intensive Therapy, University of Szeged, Szeged, Hungary
}

\section{A R T I C L E I N F O}

Article history:

Received 13 January 2014

Received in revised form 1 April 2014

Accepted 2 May 2014

Available online 9 May 2014

Keywords:

Lung mechanics

Acid aspiration

Acid aspiratio

Forced oscillations

Capnography

Mechanical ventilation

\begin{abstract}
A B S T R A C T
Bronchoaspiration results in local deterioration of lung function through direct damage and/or indirect systemic effects related to neurohumoral pathways. We distinguished these effects by selectively intubating the two main bronchi in pigs while a PEEP of 4 or $10 \mathrm{~cm} \mathrm{H}_{2} \mathrm{O}$ was maintained. Gastric juice was instilled only into the right lung. Lung mechanical and ventilation defects were assessed by measuring unilateral pulmonary input impedance $\left(Z_{\mathrm{L}, \mathrm{s}}\right)$ and the third phase slope of the capnogram $\left(S_{\mathrm{III}}\right)$ for each lung side separately before the aspiration and for 120 min thereafter. Marked transient elevations in $Z_{\mathrm{L}, \mathrm{s}}$ parameters and $S_{\text {III }}$ were observed in the affected lung after aspiration. Elevating PEEP did not affect these responses in the $Z_{\mathrm{L}, \mathrm{S}}$ parameters, whereas it prevented the $S_{\text {III }}$ increases. None of these indices changed in the intact left lung. These findings furnish evidence of the predominance of the local direct damage over the indirect systemic effects in the development of the deterioration of lung function, and demonstrate the benefit of an initially elevated PEEP following aspiration.
\end{abstract}

C 2014 Elsevier B.V. All rights reserved.

\section{Introduction}

Management of the adverse pulmonary consequences of bronchoaspiration poses a major challenge for health professionals during general anaesthesia and intensive care (Marik, 2001, 2011). This syndrome has been reported to cause a high incidence of morbidity and mortality, involving up to $90 \%$ of the affected patients, depending on the extent of the involved lung regions (Cameron et al., 1973; Engelhardt and Webster, 1999; LeFrock et al., 1979; Olsson et al., 1986; Warner et al., 1993). Inhalation of the gastric contents into the lower respiratory tract induces a number of pulmonary syndromes, described originally by Mendelson et al., including acute aspiration pneumonitis caused by the acidity subsequent to chemical injury (Mendelson, 1946), and aspiration pneumonia resulting from the inhalation of pathogenic bacteria (Marik, 2001, 2011; Raghavendran et al., 2011)

The mechanisms responsible for the acute deterioration of lung function following aspiration of the gastric contents have not been

\footnotetext{
* Corresponding author at: Department of Medical Physics and Informatics, University of Szeged, Korányi Fasor 9, H-6720 Szeged, Hungary. Tel.: +36 62 544566; fax: +3662545077 .

E-mail address: petak.ferenc@med.u-szeged.hu (F. Peták).

fully clarified. Earlier results demonstrated the involvement of direct physiochemical processes leading to mucosal damage and desquamation (Kennedy et al., 1989; Wynne et al., 1981). Another direct effect of gastric juice aspiration may be related to pepsin being cytotoxic to bronchial epithelial cells (Bathoorn et al., 2011). A further direct mechanism of lung injury following bronchoaspiration may be subsequent to the induced surfactant dysfunction (Lamonica et al., 2014). Besides these direct mechanisms, indirect pathways have also been reported to be involved in the course of aspiration, since the lung injury has been found to be mediated by capsaicin-sensitive vagal sensory afferent nerves (Kennedy et al., 1989; Kollarik et al., 2007; Martling and Lundberg, 1988; Nemzek and Kim, 2009). Another indirect route may be due to the activation of systemic inflammatory processes originating from the affected lungs, including the release of endogenous mediators by neutrophils, alveolar macrophages or by activation of the complement system leading to vascular leakage and oedema formation (Goldman et al., 1991; Kennedy et al., 1989; Knight et al., 1992; Nader-Djalal et al., 2007; Nishizawa et al., 1996; Weiser et al., 1997). Although the effective prevention and/or treatment of the bronchoaspiration would require the identification of the roles of these individual mechanisms, no previous studies have attempted to clarify the involvement of the direct and indirect pathways in the adverse functional changes in the lung during the acute phase of gastric juice inhalation. 
Another important factor that may affect the severity of the lung damage and the outcome of bronchoaspiration is the application of a raised positive end-expiratory pressure (PEEP). While an elevated PEEP is beneficial for the recruitment of lung regions and maintaining them open during mechanical ventilation (Colmenero-Ruiz et al., 1997; Ko et al., 2008), a significant haemodynamic impairment characterized by a deterioration in cardiac function may also occur during a PEEP increment (Marumo et al., 2009).

The aims of the present study were therefore to quantify the separate roles of direct and indirect mechanisms of the deterioration of lung mechanics following acid aspiration. Acidic gastric content was unilaterally administered into one lung and changes of lung mechanics were measured alternately in the affected and intact lungs. A further aim was to investigate the effects of different PEEP levels on the respiratory and circulatory outcomes during and following the inhalation of gastric juice.

\section{Materials and methods}

The work was carried out in accordance with EU Directive 2010/63/EU relating to animal experiments. The experimental protocol was approved by the institutional Animal Care Committee of the Faculty of Medicine at the University of Szeged (No. XIV/152/2013), and was performed in accordance with the National Institutes of Health guidelines for animal use.

\subsection{Animal preparations}

Male Vietnamese mini-pigs $(n=13)$ weighing $28.2 \pm 0.9 \mathrm{~kg}$ $(21-33 \mathrm{~kg})$ were used in the present study. Anaesthesia was induced by an intramuscular injection of ketamine $(20 \mathrm{mg} / \mathrm{kg}, \mathrm{CP}-$ ketamin 10\%, Produlab Pharma, Raamsdonksveer, Netherlands) and xylazine $(2 \mathrm{mg} / \mathrm{kg}$, CP-xylazin $2 \%$, Produlab Pharma, Raamsdonksveer, Netherlands) until an i.v. line was secured into the ear. A continuous infusion of propofol $(6 \mathrm{mg} / \mathrm{kg} / \mathrm{h}$, propofol $2 \% \mathrm{MCT} / \mathrm{LCT}$, Fresenius-Kabi, Bad Homburg, Germany) was then maintained throughout the study via the ear vein. The mini-pigs were tracheostomized and a double-lumen cannula (Broncho-cath right 35 Fr, Mallinckrodt Medical, Athlone, Ireland) was introduced into the distal trachea. The double lumen tracheostomy tube was positioned to achieve separate support of the left and right lung sides and the tips were sealed by inflating a cuff. The pigs were then mechanically ventilated (Model 900C; Siemens-Elema, Solna, Sweden) in volume-controlled mode with a tidal volume of $7-8 \mathrm{ml} / \mathrm{kg}$ at a frequency of $\sim 20 / \mathrm{min}$ and an inspired oxygen fraction $\left(\mathrm{FiO}_{2}\right)$ of 0.3 in air. A femoral artery was prepared surgically in a sterile manner and cannulated for blood sampling and the measurement of arterial blood pressure and cardiac output by thermodilution (PiCCO Catheters; Pulsion Medical Systems, Feldkirchen, Germany). The jugular vein was prepared in the same way as the femoral artery and cannulated for fluid and drug administration. Muscle relaxation was achieved by regular i.v. administration of pipecuronium $(0.1 \mathrm{mg} / \mathrm{kg}$, every $30 \mathrm{~min}$, Arduan, Richter-Gedeon, Budapest, Hungary). The thorax was opened by means of a midline thoracotomy following an i.v. bolus of sufentanyl $(25 \mu \mathrm{g} / \mathrm{kg}$, Sufentanil Torrex, Chiesi Pharmaceuticals, Vienna, Austria) and the ribs were widely retracted. Following chest opening, the pigs were randomly assigned to maintain a PEEP of 4 or $10 \mathrm{~cm} \mathrm{H}_{2} \mathrm{O}$.

Arterial blood samples were analyzed radiometrically (Cobas b221; Roche Diagnostics, Basel, Switzerland). The Horowitz quotient (HQ) was calculated as the ratio of the arterial partial pressure of oxygen and $\mathrm{FiO}_{2}$.

A small incision was made in the stomach and $10-20 \mathrm{ml}$ of gastric juice was obtained via a catheter introduced into the stomach. This gastric juice was filtered to remove solid particles. The $\mathrm{pH}$ of the remaining fluid was determined and hydrochloric acid was added to reach a $\mathrm{pH}$ of 2 if needed.

\subsection{Measurement of lung mechanics}

\subsubsection{Forced oscillations}

The measurement system for collection of the input impedance spectra of the right or left lung $\left(Z_{\mathrm{L}, \mathrm{S}}\right)$ in the mini-pigs was similar to that used previously for the whole lungs (Petak et al., 2006). Briefly, the mechanical ventilation was ceased at end-expiration and the cannula of the measured lung was connected to a loudspeaker-inbox system while the cannula of the other lung was occluded. Thus, during these 8-s long apnoeic periods, the forced oscillatory signal was introduced only to either the left or the right lung. Prior to the measurements, the pressure in the loudspeaker box chambers was set to the level of the PEEP to keep the mean transpulmonary pressure constant. The loudspeaker delivered a computer-generated small-amplitude $\left(< \pm 1 \mathrm{~cm} \mathrm{H}_{2} \mathrm{O}\right)$ pseudo-random signal in the interval $0.5-21 \mathrm{~Hz}$ through a screen pneumotachograph (11 mm ID), which was used to measure the gas flow $\left(V^{\prime}\right)$ with a differential pressure transducer (model 33NA002D; ICSensors, Malpitas, CA, USA) An identical pressure transducer was used to measure the pressure in the left or right main bronchi with reference to the atmosphere $\left(P_{\mathrm{L}, \mathrm{S}}\right)$.

The $P_{\mathrm{L}, \mathrm{s}}$ and $V^{\prime}$ signals were low-pass filtered at $25 \mathrm{~Hz}$ and sampled with an analogue-digital board of a microcomputer at a rate of $256 \mathrm{~Hz}$. Fast Fourier transformation with $4-\mathrm{s}$ time windows and $95 \%$ overlapping was used to calculate the unilateral $Z_{\mathrm{L}, \mathrm{s}}$ spectra $\left(Z_{\mathrm{L}, \mathrm{s}}=P_{\mathrm{L}, \mathrm{s}} / V^{\prime}\right)$.

\subsubsection{Estimation of airway and parenchymal parameters}

The airway and parenchymal mechanical properties for the individual lungs were separated by fitting a model to the $Z_{\mathrm{L}, \mathrm{S}}$ spectra by minimizing the differences between the measured and modelled impedance values. The model consisted of an airway compartment containing airway resistance (Raw) and airway inertance (law), and a constant-phase tissue unit (Hantos et al., 1992) characterized by tissue damping $(G)$ and elastance $(H)$ :

$Z_{\mathrm{L}, \mathrm{s}}=\operatorname{Raw}+j \omega \mathrm{Iaw}+\frac{G-j H}{\omega^{\alpha}}$

where $j$ is the imaginary unit, $\omega$ is the angular frequency $(2 \pi f)$, and $\alpha$ is $(2 / \pi) \arctan (H / G)$.

\subsection{Capnography}

Changes in partial $\mathrm{CO}_{2}$ pressure in the exhaled gas during mechanical ventilation were measured with a calibrated sidestream capnometer (Cardiocap II; Datex/Instrumentarium, Helsinki, Finland). $\mathrm{CO}_{2}$ traces were recorded alternately from each lung by closing one lumen of the endotracheal tube, at a time while the tidal volume was decreased by one-third by elevating the ventilation frequency. The 8-s $\mathrm{CO}_{2}$ traces obtained from the aspirated or the intact lung were imported into custom-developed signal analysis software. The third phase of the expiratory $\mathrm{CO}_{2}$ curves in each expiration was identified by selecting the $\mathrm{CO}_{2}$ traces from its peak end-tidal value back to $60 \%$ of the total length of its linear temporal change. Linear regression analysis was applied to these phases to obtain slope of the third phase of the expiratory capnogram $\left(S_{\text {III }}\right)$. $S_{\text {III }}$ was normalized by dividing each slope by the average values of the corresponding $\mathrm{CO}_{2}$ concentration in the mixed expired gas to obtain normalized time domain third phase slopes $\left(S_{\text {nIII }}\right)$. Four to six expiratory traces were analyzed in each recording. 


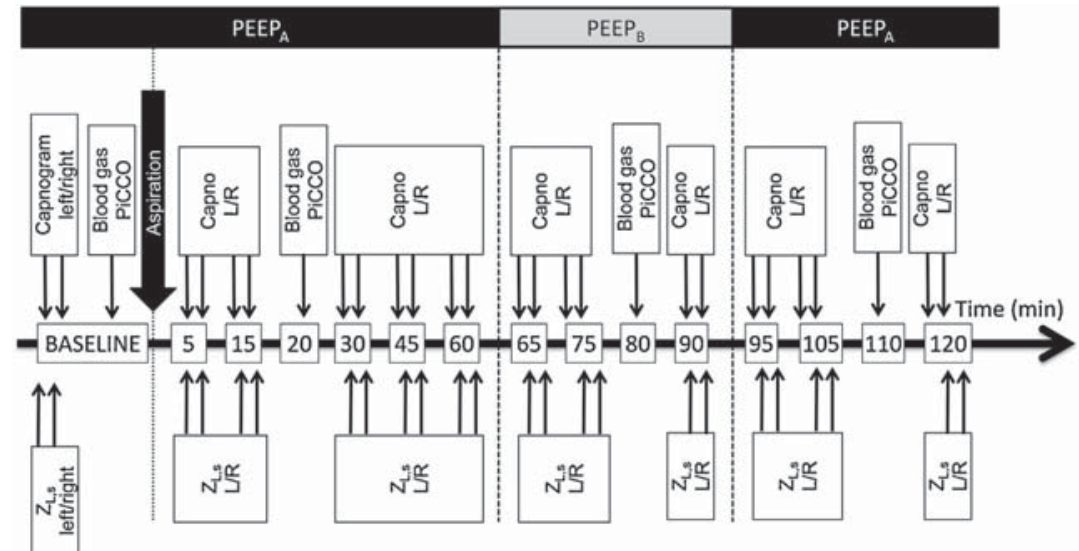

Fig. 1. Scheme of the experimental protocol. $Z_{\mathrm{L}, \mathrm{s}}$ : unilateral pulmonary input impedance measurement. $L / R$ : measurement of left or right lung. $\mathrm{PEEP}_{\mathrm{A}}$ and $\mathrm{PEEP}_{\mathrm{B}}$ correspond to 4 and $10 \mathrm{~cm} \mathrm{H}_{2} \mathrm{O}$, respectively, in random sequence.

\subsection{Haemodynamic monitoring}

Systemic haemodynamic parameters were monitored by a transpulmonary arterial thermodilution system (PiCCO; Pulsion Medical Systems, Munich, Germany). A 4F arterial catheter was inserted into the femoral artery. The thermal indicator bolus was injected into the right atrium via a central venous catheter positioned in the jugular vein. The monitor determined the cardiac index $(\mathrm{CI})$ by normalizing the cardiac output to the body surface area. The same monitoring system was used to measure the mean arterial pressure (MAP) and heart rate (HR).

\subsection{Experimental protocol}

Following stabilization of the pulmonary mechanical and haemodynamic variables after the anaesthesia and the preparation, the pigs were randomly assigned to groups in which the PEEP was set to a level of either 4 (Group P4, $n=7$ ) or $10 \mathrm{~cm} \mathrm{H}_{2} \mathrm{O}$ (Group P10, $n=6$ ). Two sets of baseline $Z_{\mathrm{L} s}$ recordings were then collected for each lung separately by forced oscillations (Fig. 1). This was followed by registration of the $\mathrm{CO}_{2}$ traces from each lung separately. Aspiration was achieved by unilaterally instilling the prepared gastric juice mixed with hydrochloric acid $(\mathrm{pH} 2)$ into the endobronchial lumen (right lung) of the tracheal tube $(0.5 \mathrm{ml} / \mathrm{kg}$ ) and leaving the left lung unaffected. To maintain the best possible gas exchange and similarity to a clinical situation, $\mathrm{FiO}_{2}$ was elevated to 1 after injection of the gastric content. Forced oscillatory and capnogram measurements were recorded for each lung alternately at $5,15,30,45$ and $60 \mathrm{~min}$ following aspiration. To assess the effect of a PEEP change in this period, the level of PEEP was interchanged between the groups ( $10-4 \mathrm{~cm} \mathrm{H}_{2} \mathrm{O}$ and $4-10 \mathrm{~cm} \mathrm{H}_{2} \mathrm{O}$, respectively) 60 min post-aspiration. Following this $P E E P$ interchange, additional $Z_{\mathrm{Ls}}$ and $\mathrm{CO}_{2}$ recordings were collected at 65,75 and $90 \mathrm{~min}$ postaspiration. Finally, PEEP was re-established at the original level and additional measurements were performed at 95, 105 and $120 \mathrm{~min}$ post-aspiration. Blood gas samples were analyzed and thermodilution measurements were performed at baseline and at 20,80 and 110 min post-aspiration.

\subsection{Statistical analysis}

The scatters in the parameters were expressed as SE values. The Kolmogorov-Smirnov test was used to test data for normality. Two-way repeated measures mixed model of analysis of variance (ANOVA) with the factors assessment time (control vs. post-aspiration) and lung side (aspirated or intact) was used in both PEEP-based groups to assess the effects of the aspiration of the gastric contents on the lung responsiveness, the slope of the third phase of the capnogram and on haemodynamic parameters. The choice of appropriate covariance structure was on the basis of Akaike information criterion measure. The Holm-Sidak multiple comparison procedure was applied to compare the different conditions (for repeated measures) or lung sides (affected or intact). Correlation analyses between the variables were performed by using Pearson correlation tests. Statistical tests were carried out with the SigmaPlot software package (version 12.5, Systat Software, Inc., CA, USA) and SAS (version 9.2, SAS Institute, Cary, NC, USA) with a significance level of $p<0.05$.

\section{Results}

There was no statistically significant difference between the weights of the pigs in the two protocol groups $(29.6 \pm 1.1 \mathrm{~kg}$ vs. $26.7 \pm 1.4 \mathrm{~kg}$ for Groups P4 and P10, respectively, $p=0.125)$. The quality of the fitting of the model to the $Z_{\mathrm{L}, \mathrm{s}}$ spectra was equally good in each group and experimental condition with mean fitting error of $6.1 \pm 0.12 \%$.

\subsection{Unilateral lung mechanical changes}

Fig. 2 demonstrates the temporal changes in the airway and lung tissue forced oscillatory mechanical parameters in both experimental groups for the affected (aspirated) and intact lungs separately. The mechanical changes following aspiration appeared generally greater in Group P4 than in P10. Under the baseline conditions, the mechanical parameters did not exhibit any statistically significant differences between the two lung sides in either experimental group. However, in both groups of pigs, significant differences developed between the two sides following gastric juice aspiration: in Raw (at least $p<0.018$ and $p<0.032$ for Groups P4 and P10, respectively), $G(p<0.05, p<0.05)$ and $H(p<0.05, p<0.036)$. The peak of the mechanical deterioration on the aspirated side occurred 5-15 min after the bronchoaspiration, with gradual improvements thereafter. The airway and tissue mechanical parameters for the intact side did not exhibit any significant change throughout the study protocol.

The changes in the lung mechanical parameters relative to their baseline values are depicted in Fig. 3 for characteristic time points 
GROUP P10
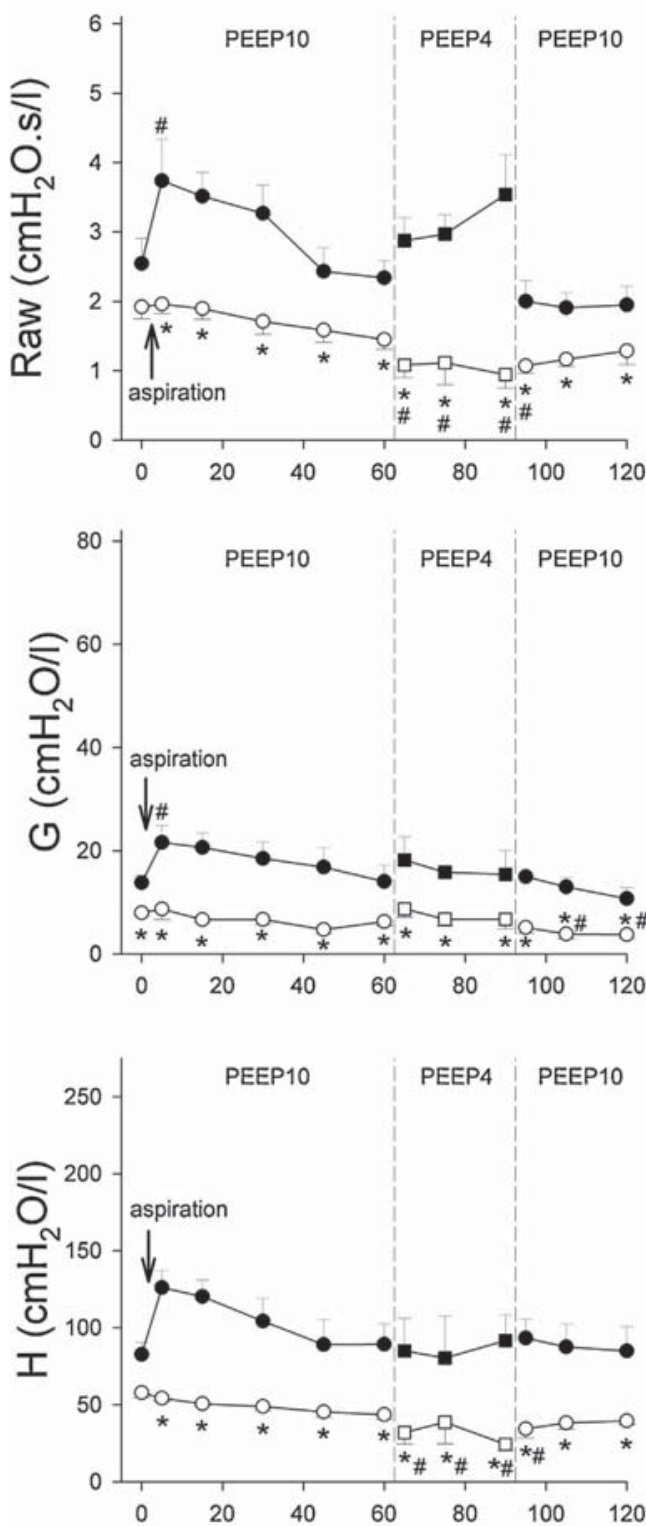

GROUP P4
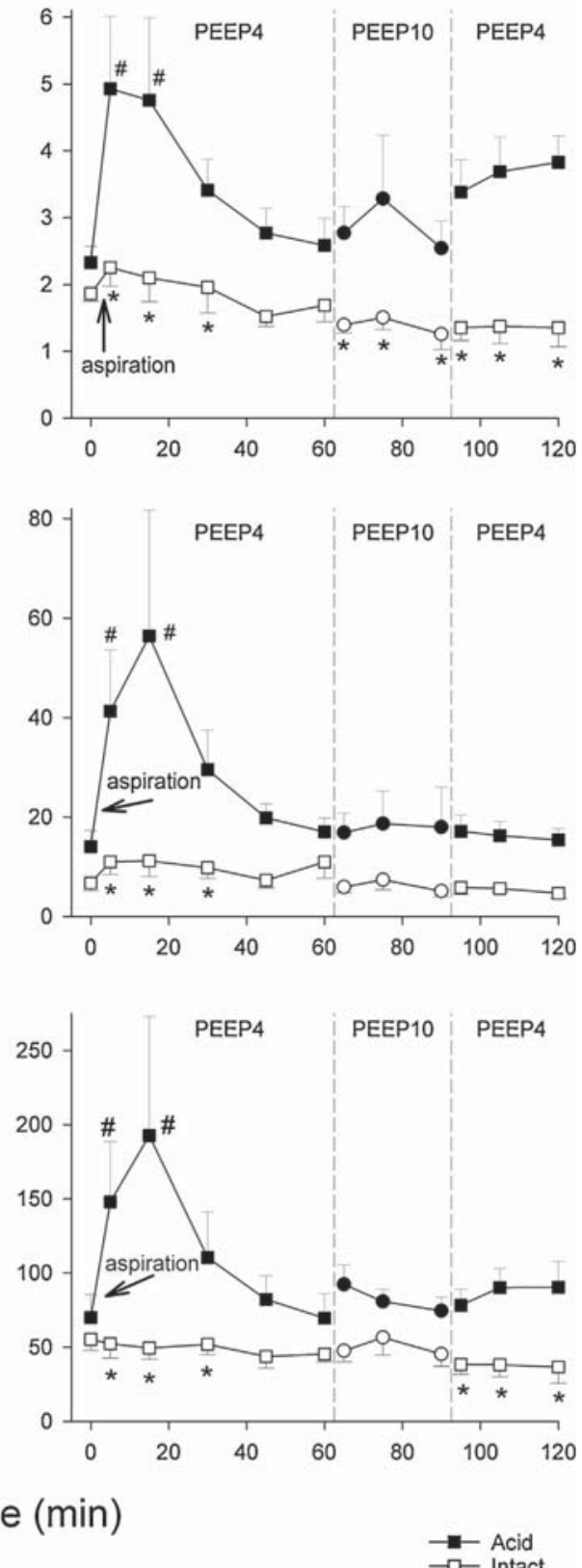

Fig. 2. Airway resistance (Raw), tissue damping $(G)$ and elastance $(H)$ for the acidic and the intact lungs in Groups P10 and P4 under baseline conditions ( 0 min) and following aspiration. " $p<0.05$ between acidic and intact lungs; ${ }^{*} p<0.05$ vs. baseline conditions within the same lung.

in the experiments. On the aspirated side, the airway and lung tissue mechanical indices were increased significantly 5 min after the aspiration in both Group P4 $(p<0.001, p=0.001$ and $p<0.001$ for Raw, $G$ and $H$, respectively) and Group P10 ( $p=0.012, p=0.007$ and $p<0.001$ ). The changes on the aspirated side were greater than those on the intact side $5 \mathrm{~min}$ after the aspiration in the pigs in Groups P4 $(p<0.014)$ and P10 $(p<0.037)$; significant differences were also apparent later for Raw and $H$ in Group P4 $(p<0.008$, $p=0.029)$ and for $G$ and $H$ in Group P10 $(p=0.037, p<0.009)$. Significant recoveries started from $60 \mathrm{~min}$ after the aspiration in all mechanical parameters. Lowering the PEEP to $4 \mathrm{~cm} \mathrm{H}_{2} \mathrm{O}$ in Group P10 caused a significant decrease in $H(p=0.002)$ on the intact side, whereas elevating the PEEP in Group P4 did not have a major effect on the unilateral lung mechanics. The unilateral lung mechanical 
GROUP P10
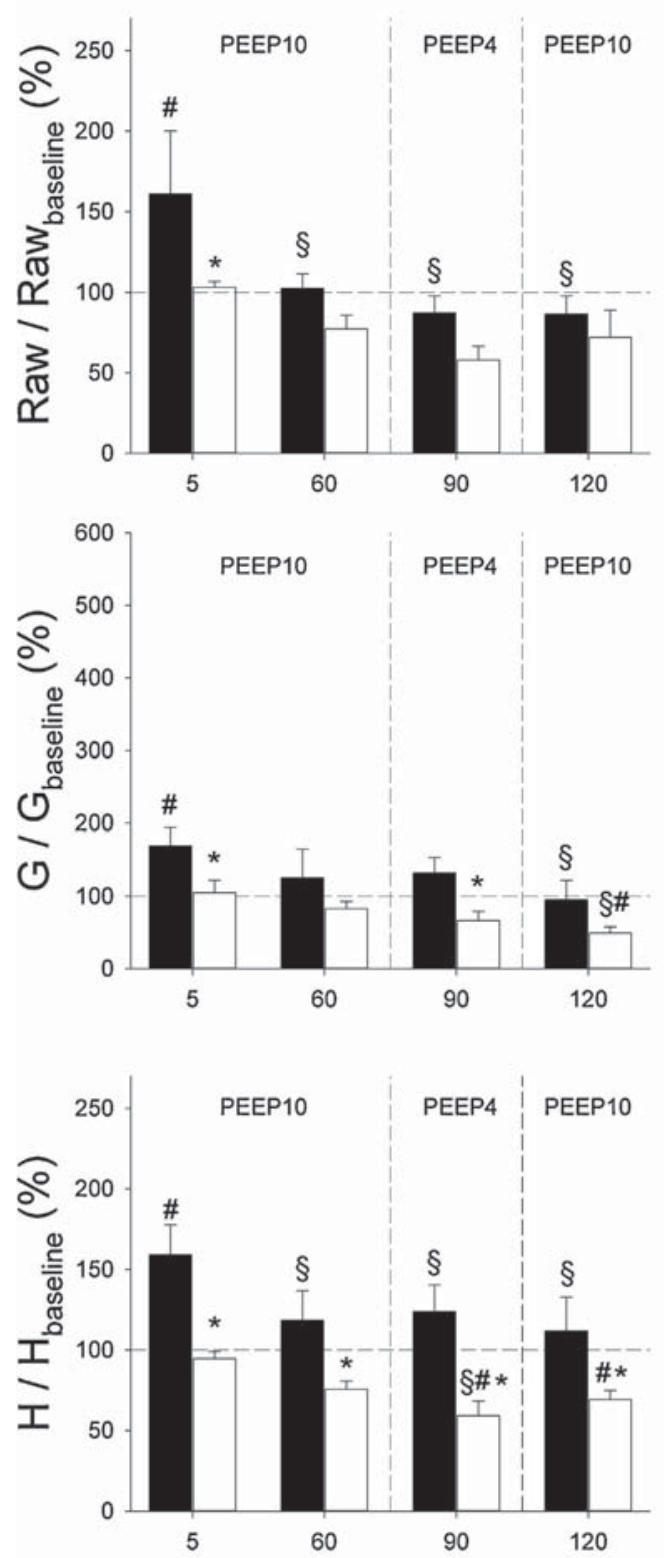

GROUP P4
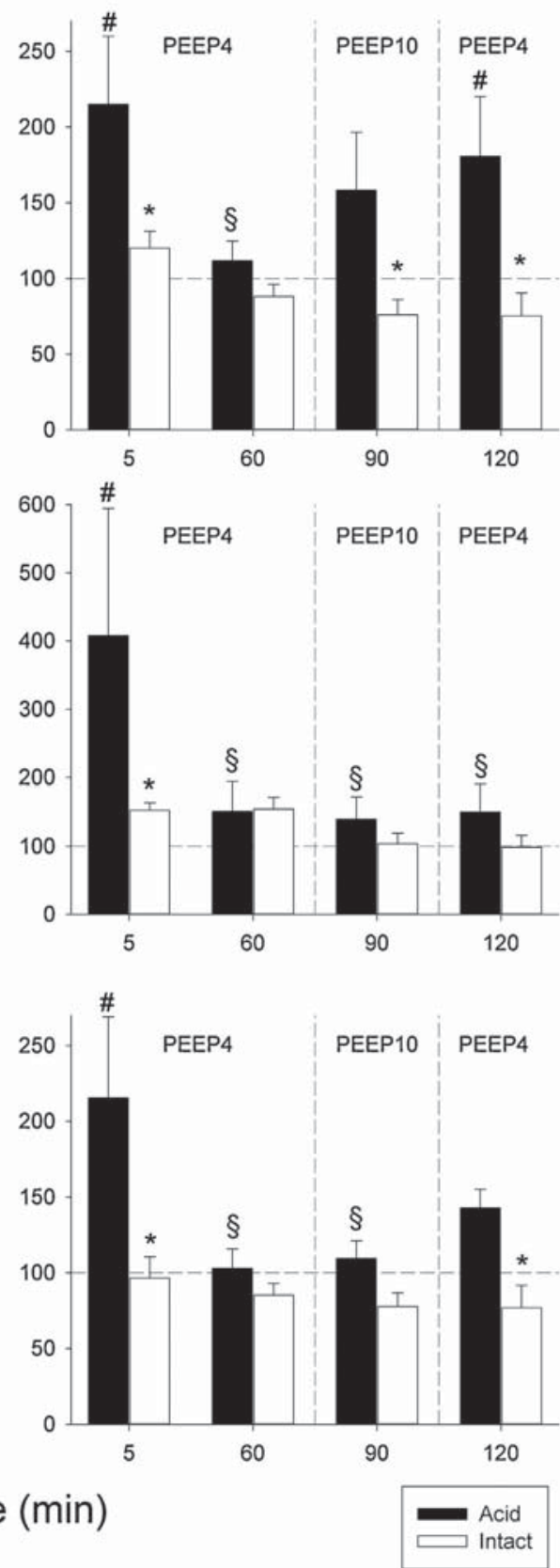

Fig. 3. Relative changes in airway resistance (Raw), tissue damping $(G)$ and elastance $(H)$ in response to bronchoaspiration at $5,60,90$ and 120 min. ${ }^{*} p<0.05$ between acidic and intact lungs; ${ }^{\#} p<0.05$ vs. baseline conditions within the same lung, $p<0.05$ vs. values at 5 min within the same lung.

parameters for the intact side did not demonstrate any significant adverse change following aspiration; in Group P10, $G$ and $H$ were even decreased on the unaffected side $120 \mathrm{~min}$ after aspiration $(p=0.021, p=0.022)$.

Since indirect mechanisms are expected to alter primarily the airway tone, the changes in Raw in the two lungs were related to reveal the relationship between the magnitudes of the bronchoconstriction. Fig 4 demonstrates the associations between the aspiration-induced changes in Raw obtained from the intact and aspirated sides for the first $60 \mathrm{~min}$ of the experiment. While fairly systematic elevations were observed in Raw on the aspirated side, the changes on the intact side were highly variable, with the 


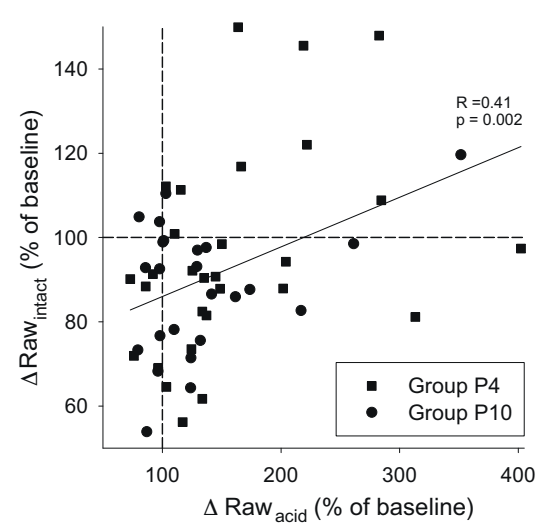

Fig. 4. Association between the changes in airway resistance in the acidic and the Fig. 4. Association between the changes in airway resistance in the acidic and the
intact lung in the first $60 \mathrm{~min}$ following aspiration in Groups P4 and P10. Solid line: linear regression.

majority of the pigs exhibiting even decrements. Nevertheless, there was a statistically significant correlation between the Raw changes on the aspirated and intact sides $(R=0.41, p=0.002)$.

\subsection{Changes in the capnogram parameters}

The changes in the absolute and normalized third phase slope of the capnogram are demonstrated in Fig. 5 under baseline conditions and 5, 60, 90 and $120 \mathrm{~min}$ after bronchoaspiration. No significant change was detected in $S_{\mathrm{III}}$ in Group P10 throughout the study, whereas $S_{\text {III }}$ was elevated at 5 min on the aspirated side in Group $\mathrm{P} 4(p=0.01)$, with an apparent recovery at $120 \mathrm{~min}(p=0.044) . S_{\mathrm{nIII}}$ did not change on the intact side during the study, whereas it was significantly elevated on the aspirated side in both groups $5 \mathrm{~min}$ after the aspiration ( $p=0.021$ and $p=0.001$ for Group P10 and P4, respectively). Changing the PEEP elevated the aspirated-side $S_{\text {nIII }}$ in the pigs of Group P10 ( $p=0.007)$, while there was no change in the intact side in the pigs of Group P10, or on either side in Group P4.

\subsection{Haemodynamic changes}

Under the baseline conditions, $\mathrm{CI}$ and HR did not differ between the protocol groups, while the MAP in Group P10 was lower than that in Group P4 (81.7 \pm 7.8 vs. $108.2 \pm 8.0 \mathrm{~mm} \mathrm{Hg}, p=0.02)$. Bronchoaspiration or the PEEP change did not lead to a significant effect on these parameters; a decrease in MAP was observed only in Group $\mathrm{P} 4$ at $110 \mathrm{~min}(108 \pm 8$ vs. $94.0 \pm 7.0 \mathrm{~mm} \mathrm{Hg}, p=0.031)$.

\subsection{Gas exchange}

Arterial blood gas parameters are demonstrated in Table 1. No significant change was observed in arterial $\mathrm{pH}$ or $\mathrm{CO}_{2}$ throughout the study protocol in either group, except for a drop in $\mathrm{pH}$ in Group P4 following the change of PEEP $(p=0.04)$, and an elevation in arterial $\mathrm{CO}_{2}$ in Group P10 at $110 \mathrm{~min}(p=0.016)$. A significant decrement was detected in HQ for both groups at $20 \mathrm{~min}(p=0.048$ and $p=0.020$ for Group P10 and Group P4, respectively), but no change was apparent thereafter.

\section{Discussion}

In the present study changes in the lung mechanical and ventilation properties were measured for the left and right lungs separately following unilateral gastric juice aspiration. This approach allowed a within-subject comparison of the local and indirect systemic effects of the bronchoaspiration by measuring the affected and the intact lung separately. Our results revealed that the marked acute lung mechanical responses observed in the airway and tissue mechanical properties in the aspirated lung were not associated with any detectable deterioration on the intact side at the 2-h follow-up after aspiration. Nevertheless, there was a significant association between the magnitude of the airway response in the intact lung and that in the aspirated lung. The presence of an uneven ventilation of the aspirated lungs was obvious from the values of the elevated normalized capnogram third phase slope. However, this change was not seen in the intact lung, in accordance with the forced oscillatory mechanical findings. The elevation of the PEEP under these conditions prevented the adverse changes in the capnogram third phase slope, while it had only minor effects on the other lung mechanical, ventilation or oxygenation indices.

In agreement with the findings of the few previous studies in which the mechanical changes were assessed for both lungs together in various animal models, we observed an acute deterioration in the lung tissue mechanics in the aspirated lung (Ko et al., 2008; Meers et al., 2011; Petak et al., 1998). This adverse change can be attributed to pulmonary oedema due to the increased vascular permeability (Goldman et al., 1993; Grimbert et al., 1981; Ko et al., 2008), surfactant dysfunction (Davidson et al., 2005; Lamonica et al., 2014) and inflammation (Knight et al., 1992; Nader-Djalal et al., 2007). Since all of these processes promote the development of atelectasis leading to a lung volume loss, the elevations in the unilateral $G$ and $H$ in the aspirated lungs are likely to occur subsequently to this phenomenon. The scarce earlier results indicated similar detrimental changes in the total resistive properties of the pulmonary system (Kuckelt et al., 1979; Meers et al., 2011; Tuchman et al., 1984). However, we are unaware of any previous studies assessing changes in airway mechanics following acid aspiration. The marked acute rise in Raw indicates the development of a transient bronchial smooth muscle contraction in the aspirated lung. Although the mechanisms responsible for this finding have not been fully clarified, it may be explained by the direct effect of the epithelial damage on the airway smooth muscle contractility (Gallos et al., 2013), or by the localized release of bronchoconstrictive neurotransmitters, such as tachykinin and bradykinin by capsaicin-sensitive neurons (Ricciardolo et al., 1999).

Respiratory abnormalities were also assessed in the present study by measuring $S_{\text {III }}$ and $S_{\text {nIII }}$. The extent of emptying of lung compartments containing various $\mathrm{CO}_{2}$ concentrations during mechanical ventilation and the shape of the resulting capnogram are determined primarily by the airway geometry (Blanch et al., 1999; Bohm et al., 2009; Nik Hisamuddin et al., 2009; Stromberg and Gustafsson, 2000; Yaron et al., 1996), and the driving pressure exerted by the elastic recoil of the respiratory tissues (Babik et al., 2012). The marked elevations in these parameters in the aspirated lungs suggest the presence of uneven lung ventilation, particularly at the lower PEEP level (Fig. 5). This may be subsequent to the localized deposition of the instilled gastric juice (Amigoni et al., 2008; Meers et al., 2011), which compromised the regional ventilation in these patchy lung areas.

Our findings also demonstrate the transient feature of this disturbance, since the ventilation abnormalities were no longer detectable $60 \mathrm{~min}$ after the aspiration. This temporal pattern suggests that the period 0-60 min is the optimum time window for the therapeutic application of an elevated PEEP against lung peripheral ventilation abnormalities following bronchoaspiration.

An elevation of the PEEP is commonly used to prevent persistent atelectasis formation following aspiration. Our results demonstrate no major benefit of this manoeuvre on either the airway or the lung tissue mechanics (Fig. 3). This finding agrees with previous results 

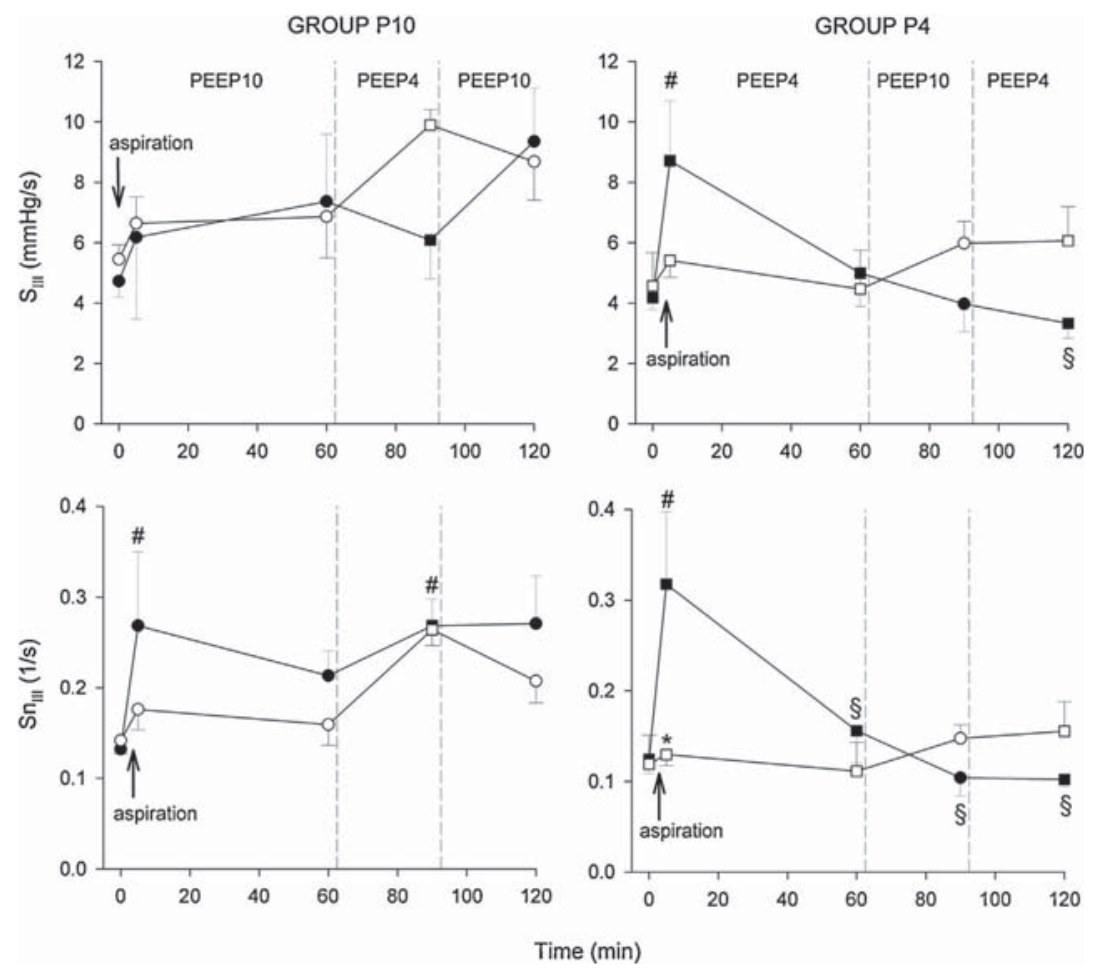

Fig. 5. Third phase slope of the capnogram ( $\left.S_{\text {III }}\right)$ and its normalized value $\left(S_{\text {nIII }}\right)$ for the acidic and the intact lungs in Groups P10 and P4 under baseline conditions ( 0 min) and following aspiration. ${ }^{*} p<0.05$ between acidic and intact lungs; ${ }^{\#} p<0.05$ vs. baseline conditions within the same lung, $p<0.05$ vs. values at 5 min within the same lung.

where no beneficial effect of a PEEP rise on the respiratory compliance was demonstrated following acid aspiration (Ko et al., 2008). However, the lack of an increase in $S_{\text {III }}$ in Group P10 indicates that the elevated PEEP prevented the development of ventilation defects in the lung periphery. This benefit was not associated with detrimental changes in the systemic haemodynamics or gas exchange. Indeed, compromised systemic haemodynamic changes have been reported only at higher PEEP levels, such as $15 \mathrm{~cm} \mathrm{H}_{2} \mathrm{O}$ (Marumo et al., 2009). This finding indicates that a moderately elevated PEEP has a beneficial effect on the aspiration-induced ventilation defects without affecting the systemic haemodynamics.

The most noteworthy finding of the present study is the experimental evidence that all these detrimental changes in the lung mechanics and ventilation were markedly repressed on the intact lung side, i.e. bronchoconstriction was detected only in the animals that responded strongly to unilateral aspiration (Fig. 4). This finding can be interpreted as it reveals a link between direct and indirect mechanisms by demonstrating the occasional presence of bronchoconstriction in the intact lung only if the bronchospasm was severe in the affected lungs. Overall, the markedly smaller responses in the intact lungs reflect the negligible role of the indirect constrictor mechanisms of systemic origin. However, it is also possible that the lack of change in the intact lungs following aspiration may result from the opposing effects of the elevated vagal tone leading to lung constriction (Kollarik et al., 2007; Nemzek and Kim, 2009) and the endogenous release of catecholamines with relaxation potentials (Coupe et al., 1987). Independently of the mechanism, our data indicate the predominance of local effects over systemic effects in the aspiration-induced deterioration of lung function.

Our observations agree with those of previous studies, where local effects prevailed in the early stage of aspiration (the first 4-6 h) (Kennedy et al., 1989). Mechanisms proposed to explain this local damage include the direct corrosive effect of the aspirated gastric content (Wynne et al., 1981), the localized acute airway inflammation caused by the pepsin in gastric juice (Bathoorn et al., 2011), the localized release of bronchoactive mediators and the subsequent injury of the alveolar epithelium (Amigoni et al., 2008). Earlier studies pointed to the systemic effects predominating in the later stages (Schreiber et al., 2006); these are related mainly to inflammatory mechanisms, such as the activation of neutrophils (Motosugi et al., 1998; Weiser et al., 1997) or alveolar macrophages.

Table

Arterial pH, Horowitz-quotient $(\mathrm{HQ})$ and arterial partial $\mathrm{CO}_{2}$ pressure in Groups $\mathrm{P} 10$ and $\mathrm{P} 4$ under baseline conditions ( 0 min) and following aspiration.

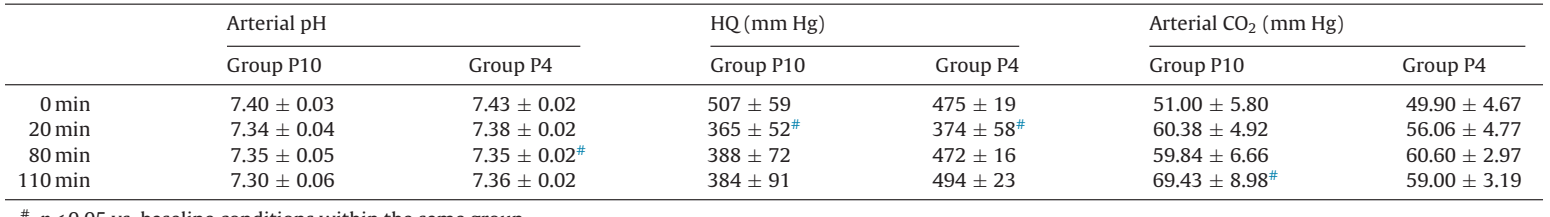


A number of methodological issues relating to the present experimental model warrant consideration. The model applied in the present study mimics the most important clinical features of bronchoaspiration, such as bronchospasm and atelectasis development leading to impaired oxygenation. However, the effects of aspiration on the lung oxygenation and on the systemic haemodynamics in the present study were less severe in magnitude than those obtained earlier (Meers et al., 2011). A plausible explanation for this apparent discrepancy may be the unilateral aspiration applied in the current experiments. This approach left one of the lungs intact, which ensured low mortality with relatively mild systemic consequences, similarly as with previously described models where unilateral acid aspiration was applied, but without assessing the functional changes in the lung in the aspirated and intact lungs individually (Amigoni et al., 2008; Schreiber et al., 2006).

A further methodological issue relates to the ventilation mode involved in our porcine model. To maintain the minute ventilation following aspiration, the volume-controlled mode was selected. However, this mode of mechanical ventilation may lead to a mild overinflation of the intact lungs following unilateral aspiration via a redistribution of the pre-set tidal volume (Amigoni et al., 2013), especially when a relatively high PEEP is applied. This phenomenon may provide a plausible explanation for the significant lowering of Raw, $G$ and $H$ in Group P10 after 60 min on the intact lung side (Figs. 2 and 3).

Another technical aspect of our experiments is related to the measurement of pulmonary mechanical parameters in the open chest without the potential confounding effects of the chest wall mechanics. Configurational differences of the lungs in the intact and open-chest may have affected the values of the airway and tissue mechanical parameters (Petak et al., 1998) and haemodynamics (Hoit et al., 1997). However, this condition was stable throughout the study protocol and the mechanical parameters were obtained during small-amplitude oscillations that minimizes the mechanical stress and thus, these factors were not likely to affect our major findings. The use of a single-compartment model to estimate airway and tissue mechanical properties may have further limitation in case of development of ventilation heterogeneities (Lutchen et al., 1996), which is expected to occur in the affected lungs following bronchoaspiration. In this case, the increases in $G$ may overestimate the real changes in the parenchymal viscoelasticity due to the involvement of an artefactual component related to enhanced ventilation inhomogeneities.

In summary, we have developed an animal model with which to distinguish the local and the systemic effects of bronchoaspiration. Our study has provided experimental evidence that unilateral aspiration of the gastric contents leads to a unilateral lung injury in the first $2 \mathrm{~h}$, characterized by acute elevations of the airway and tissue mechanical and capnogram third phase slope parameters, but exclusively on the affected side. This indicates the primary need for treatment of the local detrimental consequences of bronchoaspiration in the acute phase (the first $2 \mathrm{~h}$ ) rather than targeting the adverse systemic changes. Application of a moderately high PEEP prevented the adverse changes in the third phase slope of the capnogram, indicating decreased ventilation heterogeneities. This benefit was not associated with changes in other mechanical, cardiovascular or lung oxygenation indices, suggesting that a moderately high PEEP may be beneficial in the optimum lung management following bronchoaspiration.

\section{Acknowledgements}

The study was supported by a Hungarian Scientific Research Grant (OTKA K81179).
This research was supported by the European Union and the State of Hungary, co-financed by the European Social Fund in the framework of TÁMOP 4.2.4.A/2-11-1-2012-0001 'National Excellence Program' and 4.2.2.A-11/1/KONV-2012-0052.

The authors thank József Kaszaki for his excellent advice and Gabriella Varga for her invaluable assistance during the experiments.

The authors are grateful to Katalin Virág for the statistical advices.

\section{References}

Amigoni, M., Bellani, G., Scanziani, M., Masson, S., Bertoli, E., Radaelli, E., Patroniti, N., Di Lelio, A., Pesenti, A., Latini, R., 2008. Lung injury and recovery in a murine model of unilateral acid aspiration: functional, biochemical, and morphologic characterization. Anesthesiology 108, 1037-1046.

Amigoni, M., Bellani, G., Zambelli, V., Scanziani, M., Farina, F., Fagnani, L., Latini, R. Fumagalli, R., Pesenti, A., 2013. Unilateral acid aspiration augments the effects of ventilator lung injury in the contralateral lung. Anesthesiology 119, 642-651.

Babik, B., Csorba, Z., Czovek, D., Mayr, P.N., Bogats, G., Petak, F., 2012. Effects of respiratory mechanics on the capnogram phases: importance of dynamic compliance of the respiratory system. Crit. Care 16, R177.

Bathoorn, E., Daly, P., Gaiser, B., Sternad, K., Poland, C., Macnee, W., Drost, E.M. 2011. Cytotoxicity and induction of inflammation by pepsin in acid in bronchial epithelial cells. Int. J. Inflam., 569416.

Blanch, L., Lucangelo, U., Lopez-Aguilar, J., Fernandez, R., Romero, P.V., 1999. Volumetric capnography in patients with acute lung injury: effects of positive end-expiratory pressure. Eur. Respir. J. 13, 1048-1054.

Bohm, S.H., Maisch, S., von Sandersleben, A., Thamm, O., Passoni, I., Martinez Arca, J., Tusman, G., 2009. The effects of lung recruitment on the Phase III slope of volumetric capnography in morbidly obese patients. Anesth. Analg. 109, 151-159.

Cameron, J.L., Mitchell, W.H., Zuidema, G.D., 1973. Aspiration pneumonia. Clinical outcome following documented aspiration. Arch. Surg. 106, 49-52.
out

Colmenero-Ruiz, M. Fernandez-Mondejar, E. Fernandez-Sacristan, M.A., RiveraFernandez, R., Vazquez-Mata, G., 1997. PEEP and low tidal volume ventilation reduce lung water in porcine pulmonary edema. Am. J. Respir. Crit. Care Med. reduce lung wa $964-970$.

Coupe, M.O., Guly, U., Brown, E., Barnes, P.J., 1987. Nebulised adrenaline in acute severe asthma: comparison with salbutamol. Eur. J. Respir. Dis. 71, 227-232.

Davidson, B.A., Knight, P.R., Wang, Z., Chess, P.R., Holm, B.A., Russo, T.A., Hutson, A., Notter, R.H., 2005. Surfactant alterations in acute inflammatory lung injury from aspiration of acid and gastric particulates. Am. J. Physiol. Lung Cell Mol. Physiol. 288, L699-L708.

Engelhardt, T., Webster, N.R., 1999. Pulmonary aspiration of gastric contents in anaesthesia. Br. J. Anaesth. 83, 453-460

Gallos, G., Townsend, E., Yim, P., Virag, L., Zhang, Y., Xu, D., Bacchetta, M., Emala, C.W. 2013. Airway epithelium is a predominant source of endogenous airway GABA and contributes to relaxation of airway smooth muscle tone. Am. J. Physiol. Lung Cell Mol. Physiol. 304, L191-L197.

Goldman, G., Welbourn, R., Klausner, J.M., Kobzik, L., Valeri, C.R., Shepro, D., Hechtman, H.B., 1991. Neutrophil accumulations due to pulmonary thromboxane synthesis mediate acid aspiration injury. J. Appl. Physiol. 70, 1511-1517.

Goldman, G., Welbourn, R., Klausner, J.M., Kobzik, L., Valeri, C.R., Shepro, D., Hechtman, H.B., 1993. Leukocytes mediate acid aspiration-induced multiorgan edema. Surgery $114,13-20$.

Grimbert, F.A., Parker, J.C., Taylor, A.E., 1981. Increased pulmonary vascular permeability following acid aspiration. J. Appl. Physiol. 51, 335-345.

Hantos, Z., Daroczy, B., Suki, B., Nagy, S., Fredberg, J.J., 1992. Input impedance and peripheral inhomogeneity of dog lungs. J. Appl. Physiol. 72, 168-178.

Hoit, B.D., Ball, N., Walsh, R.A., 1997. Invasive hemodynamics and force-frequency relationships in open- versus closed-chest mice. Am. J. Physiol. 273, H2528-H2533.

Kennedy, T.P., Johnson, K.J., Kunkel, R.G., Ward, P.A., Knight, P.R., Finch, J.S., 1989. Acute acid aspiration lung injury in the rat: biphasic pathogenesis. Anesth. Analg. 69, 87-92

Knight, P.R., Druskovich, G., Tait, A.R., Johnson, K.J., 1992. The role of neutrophils, oxidants, and proteases in the pathogenesis of acid pulmonary injury. Anesthesiology $77,772-778$

Ko, S.C., Zhang, H., Haitsma, J.J., Cheng, K.C., Li, C.F., Slutsky, A.S., 2008. Effects of PEEP levels following repeated recruitment maneuvers on ventilator-induced lung injury. Acta Anaesthesiol. Scand. 52, 514-521.

Kollarik, M., Ru, F., Undem, B.J., 2007. Acid-sensitive vagal sensory pathways and cough. Pulm. Pharmacol. Ther. 20, 402-411.

Kuckelt, W., Dauberschmidt, R., Bender, V., Hieronymi, U., Mrochen, H., Winsel, K. Althaus, P., Meyer, M., 1979. Gas exchange, pulmonary mechanics and haemodynamics in adult respiratory distress syndrome: experimental results in Lew miniature pigs. Resuscitation 7, 13-33.

Lamonica, G., Amigoni, M., Vedovelli, L., Zambelli, V., Scanziani, M., Bellani, G., Grassi, A., Simonato, M., Carnielli, V.P., Cogo, P.E., 2014. Pulmonary surfactant synthesi after unilateral lung injury in mice. J. Appl. Physiol. (1985) 116, 210-215.

LeFrock, J.L., Clark, T.S., Davies, B., Klainer, A.S., 1979. Aspiration pneumonia: a tenyear review. Am. Surg. 45, 305-313. 
Lutchen, K.R., Hantos, Z., Petak, F., Adamicza, A., Suki, B., 1996. Airway inhomogeneities contribute to apparent lung tissue mechanics during constriction. J. Appl. Physiol. 80, 1841-1849.

Marik, P.E., 2001. Aspiration pneumonitis and aspiration pneumonia. N. Engl. J. Med. 344, 665-671.

Marik, P.E., 2011. Pulmonary aspiration syndromes. Curr. Opin. Pulm. Med. 17, $148-154$.

Martling, C.R., Lundberg, J.M., 1988. Capsaicin sensitive afferents contribute to acute airway edema following tracheal instillation of hydrochloric acid or gastric juice in the rat. Anesthesiology 68, 350-356

Marumo, C.K., Otsuki, D.A., Fantoni, D.T., Margarido, C.B., Ambrosio, A.M., Pelosi, P., Auler Jr., J.O., 2009. Hemodynamic effects of PEEP in a porcine model of $\mathrm{HCl}-$ induced mild acute lung injury. Acta Anaesthesiol. Scand. 53, 190-202.

Meers, C.M. De Wever, W., Verbeken, E., Mertens, V., Wauters, S., De Vleeschauwer, S.I., Vos, R., Vanaudenaerde, B.M., Verleden, G.M., Van Raemdonck, D.E., 2011 A porcine model of acute lung injury by instillation of gastric fluid. J. Surg. Res.

Mendelson, C.L., 1946. The aspiration of stomach contents into the lungs during obstetric anesthesia. Am. J. Obstet. Gynecol. 52, 191-205

Motosugi, H., Quinlan, W.M., Bree, M., Doerschuk, C.M., 1998. Role of CD11b in focal acid-induced pneumonia and contralateral lung injury in rats. Am. J. Respir. Crit. Care Med. 157, 192-198.

Nader-Djalal, N., McQuiller, P.S., Raghavendran, K., Spengler, R.N., Knight, P.R., 2007. The role of alveolar macrophages in the pathogenesis of aspiration pneumonitis. Immunol. Invest. 36, 457-471.

Nemzek, J.A., Kim, J., 2009. Pulmonary inflammation and airway hyperresponsivehess in a mouse model of asthma complicated by acid aspiration. Comp. Med. 59, 321-330.

Nik Hisamuddin, N.A., Rashidi, A., Chew, K.S., Kamaruddin, J., Idzwan, Z., Teo, A.H. 2009. Correlations between capnographic waveforms and peak flow meter measurement in emergency department management of asthma. Int. J. Emerg. Med. $2,83-89$

Nishizawa, H., Yamada, H., Miyazaki, H., Ohara, M., Kaneko, K., Yamakawa, T., Wiener-Kronish, J., 1996. Soluble complement receptor type 1 inhibited the systemic organ injury caused by acid instillation into a lung. Anesthesiology $85,1120-1128$.
Olsson, G.L., Hallen, B., Hambraeus-Jonzon, K., 1986. Aspiration during anaesthesia: a computer-aided study of 185,358 anaesthetics. Acta Anaesthesiol. Scand. 30, $84-92$.

Petak, F., Habre, W., Babik, B., Tolnai, J., Hantos, Z., 2006. Crackle-sound recording to monitor airway closure and recruitment in ventilated pigs. Eur. Respir. J. 27, 808-816.

Petak, F., Hall, G.L., Sly, P.D., 1998. Repeated measurements of airway and parenchymal mechanics in rats by using low-frequency oscillations. J. Appl. Physiol. (1985) $84,1680-1686$.

Raghavendran, K., Nemzek, J., Napolitano, L.M., Knight, P.R., 2011. Aspirationinduced lung injury. Crit. Care Med. 39, 818-826.
indurat.,

Ricciardolo, F.L., Rado, V., Fabbri, L.M., Sterk, P.J., Di Maria, G.U., Geppetti, P., 1999. Bronchoconstriction induced by citric acid inhalation in guinea pigs: role of tachykinins, bradykinin, and nitric oxide. Am. J. Respir. Crit. Care Med. 159, 557-562.

Schreiber, T., Hueter, L., Gaser, E., Schmidt, B., Schwarzkopf, K., Rek, H., Karzai, W., 2006. PEEP has beneficial effects on inflammation in the injured and no deleterious effects on the noninjured lung after unilateral lung acid instillation. Intens. Care Med. 32, 740-749.

Stromberg, N.O., Gustafsson, P.M., 2000. Ventilation inhomogeneity assessed by nitrogen washout and ventilation-perfusion mismatch by capnography in stable and induced airway obstruction. Pediatr. Pulmonol. 29, 94-102.

Tuchman, D.N., Boyle, J.T., Pack, A.I., Scwartz, J., Kokonos, M., Spitzer, A.R., Cohen, S., 1984. Comparison of airway responses following tracheal or esophageal acidification in the cat. Gastroenterology 87, 872-881.

Warner, M.A., Warner, M.E., Weber, J.G., 1993. Clinical significance of pulmonary aspiration during the perioperative period. Anesthesiology 78, 56-62.

Weiser, M.R., Pechet, T.T., Williams, J.P., Ma, M., Frenette, P.S., Moore, F.D., Kobzik, L., Hines, R.O., Wagner, D.D., Carroll, M.C., Hechtman, H.B., 1997. Experimental murine acid aspiration injury is mediated by neutrophils and the alternative complement pathway. J. Appl. Physiol. 83, 1090-1095.

Wynne, J.W., Ramphal, R., Hood, C.I., 1981. Tracheal mucosal damage after aspiration. A scanning electron microscope study. Am. Rev. Respir. Dis. 124, 728-732.

Yaron, M., Padyk, P., Hutsinpiller, M., Cairns, C.B., 1996. Utility of the expiratory capnogram in the assessment of bronchospasm. Ann. Emerg. Med. 28, 403-407. 


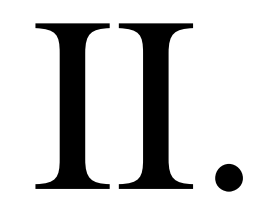

Fodor G.H., Babik B., Czövek D., Doras C., Balogh Á.L., Bayat S., Habre W., Peták F.: Effects of fluid replacement on respiratory function: comparison of whole blood with colloid and crystalloid. Eur J Anaesthesiol. 2016; 33:34-41 


\title{
ORIGINAL ARTICLE
}

\section{Fluid replacement and respiratory function: comparison of whole blood with colloid and crystalloid}

\author{
$A$ randomised animal study \\ Gergely H. Fodor, Barna Babik, Dorottya Czövek, Camille Doras, Ádám L. Balogh, \\ Sam Bayat, Walid Habre and Ferenc Peták
}

BACKGROUND Fluid replacement with blood products, colloids and crystalloids is associated with morbidity and mortality. Despite this, the consequences of fluid administration on airway and respiratory tissue properties are not fully understood.

OBJECTIVE Comparison of respiratory effects of fluid replacement with autologous blood (Group B), colloid (HES 6\% $130 / 0.4$, Group $\mathrm{CO}$ ) or crystalloid solution ( $\mathrm{NaCl} 0.9 \%$, Group CR) after haemorrhage with separate assessments of airway resistance and respiratory tissue mechanics.

DESIGN A randomised study.

SETTING An experimental model of surgical haemorrhage and fluid replacement in rats.

PARTICIPANTS Anaesthetised, ventilated rats randomly allocated into three groups (Group B: $n=8$, Group CO: $n=8$, Group CR: $n=9$ ).

INTERVENTION Animals were bled in six sequential steps, each manoeuvre targeting a loss of $5 \%$ of total blood volume. The blood loss was then replaced stepwise in a $1: 1$ ratio with one of the three fluids.

MAIN OUTCOME MEASURE After each step, airway resistance (Raw), tissue damping and elastance $(H)$ were determined by forced oscillations. Oedema indices from lung weights and histology were also measured.

RESULTS Raw (mean \pm SD) decreased in all groups following blood loss $(-20.3 \pm 9.5 \%$ vs. baseline, $P<0.05)$, and remained low following blood replacement $(-21.7 \pm 14.5 \%$ vs. baseline, $P<0.05)$, but was normalised by colloid $(5.5 \pm 10.7 \%, N S)$. Crystalloid administration exhibited an intermediate reversal effect $(-8.4 \pm 14.7 \%, N S)$. Tissue viscoelasticity increased following both blood loss and replacement, with no evidence of a significant difference in $\mathrm{H}$ between Groups $\mathrm{CO}$ and $\mathrm{CR}$. More severe oedema was observed in Groups CR and CO than in Group B $(P<0.05)$, with no difference between the colloid and crystalloid solutions.

CONCLUSION This model, which mimics surgical haemorrhage, yields no evidence of a difference between colloids and crystalloids with regard to the pulmonary consequences of blood volume restoration. Functional changes in the lung should not be a key concern when choosing fluid replacement therapy with these solutions.

Published online 10 March 2015

\section{Introduction}

Blood loss during major surgery is associated with detrimental systemic and pulmonary effects. The best fluid replacement management following haemorrhage is one of the most polarising issues in anaesthesia and intensive therapy. Choosing the best fluid replacement from blood products, and various types of colloids and crystalloids, can be difficult. The safety of hydroxyethyl-starch (HES) has been brought into question in a recent meta-analysis, ${ }^{1}$

From the Department of Medical Physics and Informatics (GHF, DC, FP), Department of Anaesthesiology and Intensive Therapy, University of Szeged, Szeged, Hungary (BB, ÁLB), Pathophysiological Experimental Platform, Department of Anaesthesiology, Pharmacology and Intensive Care, University of Geneva (CD), Paediatric Anaesthesia Unit, Geneva Children's Hospital, University Hospitals of Geneva, Geneva, Switzerland (WH), and Université de Picardie Jules Verne, Inserm U1105 Anaesthesia Unit, Geneva Children's Hospital, University Hospitals of Geneva,
and Paediatric Lung Function Laboratory, CHU Amiens, Amiens, France (SB)

Correspondence to Barna Babik, MD, PhD, Department of Anaesthesiology and Intensive Therapy, University of Szeged, 6 Semmelweis str, Szeged H-6725, Hungary Tel: +36 62545 168; fax: +36 62545 593; e-mail: babikbarna@gmail.com 
particularly in patients with increased capillary leakage. This and the risk of renal damage with HES, ${ }^{2,3}$ the appreciable cost of albumin and the defects of haemostasis induced by gelatin solutions have all reduced the available options. ${ }^{4}$ Crystalloids remain a rational option, but clinicians are reluctant to choose them because of the widespread belief that they move rapidly out of the circulation, a belief based on old studies that lacks an adequate evidence basis. ${ }^{5-7}$

We have recently shown that acute hypovolaemic shock and subsequent resuscitation with autologous blood affects respiratory mechanics. ${ }^{8}$ Although surgical procedures are associated with a more controlled but sustained blood loss that also requires fluid replacement therapy, the respiratory consequences of this have not been explored. The administration of blood products is often regarded as the gold standard therapy, with the main aim of maintaining oxygen transport capacity. However, no evidence-based data are available to allow a comparison of the changes in lung function between this consensual approach and goal-directed fluid therapy with colloids or crystalloids. This experimental study uses a novel animal model that mimics continuous, hidden surgical bleeding and replacement of the associated losses. We aimed to compare the effects of blood, colloid and crystalloid solutions on the flow resistance of the airways and on the viscoelastic properties of the respiratory tissues and then attempted to relate these changes to pulmonary oedema indices. We hypothesised that the respiratory effects of fluid resuscitation with blood would differ from those observed after colloid and crystalloid solutions.

\section{Materials and methods}

Ethical approval for this study (no. I-74-50/2012) was provided by the Experimental Ethics Committee of the University of Szeged, Szeged, Hungary, on 7 December 2012, and granted by the Animal Health and Welfare Office of the local authorities in Hungary (no. XIV/152/ 2013, Chairperson Cs. Farle) on 9 January 2013.

\section{Animal preparation}

Anaesthesia was induced with an intraperitoneal injection of $5 \%$ chloral hydrate $\left(400 \mathrm{mg} \mathrm{kg}^{-1}\right)$ in adult male Sprague Dawley rats. Tracheal intubation was achieved with a 16-gauge, polyethylene cannula (B. Braun Melsungen AG, Melsungen, Germany) after subcutaneous administration of local anaesthetic to ensure adequate analgesia around the surgical wound (lidocaine, 2 to $4 \mathrm{mg} \mathrm{kg}^{-1}$ ). The rats were then placed in a supine position on a heating pad and connected to a small animal ventilator (Model 683; Harvard Apparatus, South Natick, Massachusetts, USA), and mechanically ventilated with room air $\left(70\right.$ breaths $\min ^{-1}$, tidal volume $\left.7 \mathrm{ml} \mathrm{kg}^{-1}\right)$. A femoral vein was catheterised (Abbocath 22-gauge) for drug delivery and for fluid replacement. A femoral artery was cannulated (Abbocath 22-gauge) and attached to a pressure transducer (Model TSD104A; Biopac, Santa Barbara, California, USA) for continuous monitoring of mean arterial pressure (MAP), and to allow blood withdrawal, as part of the experimental protocol. The arterial pressure, ECG and heart rate (HR) were monitored continuously with a data collection and acquisition system (Biopac). Body temperature was kept in the $37 \pm 0.5^{\circ} \mathrm{C}$ range by using the heating pad.

\section{Measurement of respiratory mechanics}

The forced oscillation technique was applied in short (6-s long) end-expiratory pauses interposed in the mechanical ventilation sequence to measure the input impedances of the respiratory system $(\mathrm{Zrs}){ }^{9}$ Briefly, the ventilator was stopped at end-expiration and the tracheal cannula was switched from the ventilator to a loudspeaker-in-box system. The loudspeaker delivered a computer-generated small-amplitude $\left(<1 \mathrm{cmH}_{2} \mathrm{O}\right)$ pseudorandom signal (23 noninteger multiples between 0.5 and $21 \mathrm{~Hz}$ ) through a 100-cm long, 2-mm internal diameter polyethylene tube. Two identical pressure transducers (model 33NA002D; ICSensors, Milpitas, California, USA) were used to measure the lateral pressures at the loudspeaker end $\left(P_{1}\right)$ and at the tracheal end $\left(P_{2}\right)$ of the wave-tube. The signals $P_{1}$ and $P_{2}$ were low-pass filtered (fifth-order Butterworth, 25-Hz corner frequency) and sampled with the analogue-digital board of a microcomputer at a rate of $256 \mathrm{~Hz}$. Fast Fourier transformation with 4-s time windows and 95\% overlapping was used to calculate the pressure transfer functions $\left(P_{1} / P_{2}\right)$ from the 6-s recordings collected during apnoea. Zrs was calculated as the load impedance of the wave-tube. ${ }^{10}$ The input impedances of the tracheal tube and the connections were also determined and subtracted from each Zrs spectrum.

A model containing a frequency-independent resistance $(R)$ and inertance $(I)$, and tissue damping $(G)$ and elastance $(H)$ of a constant-phase tissue compartment ${ }^{11}$ was fitted to the Zrs spectra by minimising the weighted difference between the measured and the modelled impedance data. The tissue variables characterise the damping (resistive) and elastic properties of the respiratory system. Raw and Iaw represent primarily the resistance and inertance of the airways, as the contribution of the chest wall to these variables in rats is minor. ${ }^{12}$

\section{Lung histology}

After completion of the experimental protocol, the rats were euthanised with an overdose of intravenous (i.v.) pentobarbital sodium $\left(300 \mathrm{mg} \mathrm{kg}^{-1}\right)$. Midline thoracotomy was then performed and $4 \%$ formaldehyde was instilled into the right lung via the tracheal cannula at a hydrostatic pressure of $20 \mathrm{cmH}_{2} \mathrm{O}$ after clamping of the left main bronchus near the bifurcation. The right lung was dissected and placed into $4 \%$ buffered formalin until further processing. After complete fixation, horizontal 
trans-hilar sections (perpendicular to the longitudinal axes of the lung from the hilum) were embedded in paraffin. Two $5 \mu \mathrm{m}$ sections were prepared in each lung specimen and were stained with haematoxylin-eosin. Digitalised images were used to obtain the oedema index around randomly selected pulmonary vessels by dividing the lumen area by the total area of the pulmonary vessel (oedema cuff area + vessel lumen area). Histological images were analysed by the same investigator in a blind fashion and in a random sequence by using JMicroVision image analysis software (version 1.2.7).

Three to four tissue samples were dissected from the different lobes of the unfixed left lungs; these samples were weighed to establish the wet-to-dry weight ratio $(\mathrm{W} / \mathrm{D})$ as an index of the lung water content.

\section{Experimental protocol}

The rats were randomly assigned into one of the three protocol groups. The rats in Group B always received autologous heparinised blood $(n=8)$. Fluid replacement was performed with a colloid solution (HES 6\% 130/0.4; Fresenius Kabi Deutschland GmbH, Bad Homburg v.d.H., Germany) in Group CO $(n=8)$, or with a crystalloid solution ( $\mathrm{NaCl} 0.9 \%$; B. Braun Melsungen AG, Melsungen, Germany) in Group CR $(n=9)$. The experiment began with standardisation of the lung volume history through the administration of a hyperinflation created by occluding the expiratory port of the ventilator once the animal had reached a steady-state condition (5 to $10 \mathrm{~min}$ after starting mechanical ventilation). The baseline respiratory mechanics were then established by measuring three or four reproducible Zrs data epochs. Next, haemorrhage was induced by the withdrawal of $5 \%$ of the estimated total blood volume ${ }^{13}$ via the femoral artery (Fig. 1). Three minutes later, another set of Zrs data was collected, including three individual measurements at 1-min intervals. The withdrawn blood was used for blood gas analyses (Cobas b221; Roche Diagnostics, Basel, Switzerland) to determine the haematocrit (Hct), $\mathrm{pH}$ and oxygen $\left(p a \mathrm{O}_{2}\right)$ and carbon dioxide $\left(p a \mathrm{CO}_{2}\right)$ partial pressures. The blood withdrawal and Zrs measurements were repeated once more in an identical manner. After completion of the first two steps of arterial haemorrhage, fluid replacement in accordance with the group allocation was performed by administering $5 \%$ of the total blood volume via the femoral vein. Three minutes after this manoeuvre, a set of Zrs data was recorded. The blood withdrawal/replacement procedure was repeated four more times, with the collection of Zrs data 3 min after each intervention. The total duration of resuscitation was around $90 \mathrm{~min}$, with each step lasting approximately $7 \mathrm{~min}$. Further arterial blood gas analyses were performed from the fourth and sixth blood samples. After completion of the measurement protocol, the lungs were processed for oedema assessment, as detailed above.

\section{Data analysis}

The scatters in the measured variables were expressed as $\mathrm{SD}$ values. The Kolmogorov-Smirnov test was used to test data for normality. Two-way repeated measures of analysis of variance (ANOVA), with the factors assessment time and group allocation, were used to assess the effects of blood loss and replacement on the respiratory mechanical and haemodynamic variables. The baseline respiratory mechanical assessments and oedema indices were compared by using one-way ANOVA. The HolmSidak multiple comparison procedure was applied to compare the different conditions (for repeated measures) or protocol groups (for independent groups). Correlation analyses between the variables were performed by using Pearson correlation tests. Statistical tests were carried out with the SigmaPlot software package (version 12.5; Systat Software, Inc., California, USA) with a significance level of $P$ value of less than 0.05 .

\section{Results}

Rat body weights were not statistically different between the groups $(344 \pm 16.1 \mathrm{~g}$ for Group B, $320 \pm 51.24 \mathrm{~g}$ for Group CO and $361 \pm 20.7 \mathrm{~g}$ for Group CR). Table 1 summarises the baseline values of the respiratory mechanics for the three experimental groups. No statistically significant

Fig. 1

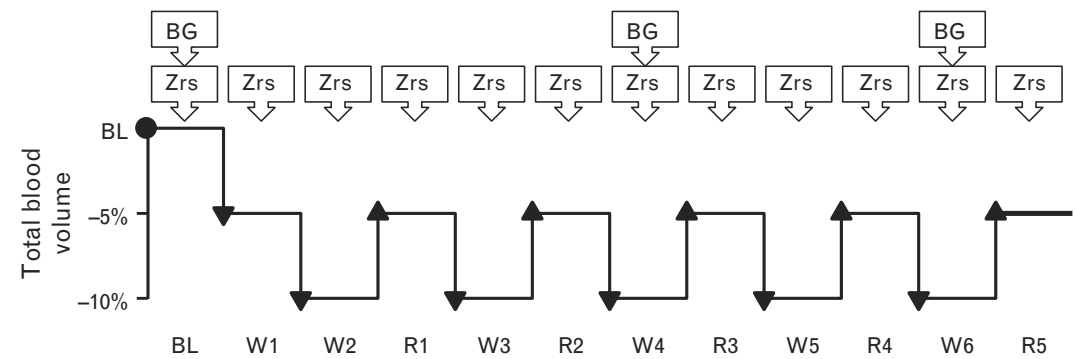

Scheme of the experimental protocol. BG, assessment of arterial blood gas; BL, baseline; R1 to R5, fluid replacements; W1 to W6, blood withdrawals; Zrs, measurement of respiratory impedance data.

\section{Eur J Anaesthesio/ 2016; 33:34-41 \\ Copyright @ 2015 Wolters Kluwer Health, Inc. All rights reserved.}


Table 1 Mean (SD) values of the airway resistance (Raw), tissue damping $(G)$ and elastance $(H)$ obtained under the baseline conditions in the three groups of rats

\begin{tabular}{lccc} 
& $\begin{array}{c}\text { Raw } \\
\left(\mathbf{c m H}_{\mathbf{2}} \mathbf{O} \cdot \mathbf{s} \cdot \mathbf{I}^{-1}\right)\end{array}$ & $\begin{array}{c}\mathbf{G} \\
\left(\mathbf{c m H}_{\mathbf{2}} \mathbf{O I}^{-1}\right)\end{array}$ & $\begin{array}{c}\mathbf{H} \\
\left(\mathrm{cm} \mathrm{H}_{\mathbf{2}} \mathbf{O I}^{-1}\right)\end{array}$ \\
Group B & $54.4(7.6)$ & $1034(93.2)$ & $5332(761.6)$ \\
Group CO & $52.3(10.9)$ & $1061(131.3)$ & $5293(1139.6)$ \\
Group CR & $51.7(9.9)$ & $912(105.0)$ & $4533(546.0)$ \\
\hline
\end{tabular}

differences were detected in the variables reflecting the airway or tissue mechanics.

The arterial blood gas values obtained at the beginning, at the midpoint and at the end of the experimental protocol are presented in Table 2. In Group B, Hct did not change significantly throughout the protocol, whereas decreases in $\mathrm{pH} \quad(P<0.001)$ and $\mathrm{paO}_{2}$ $(P=0.011)$ were seen. Compared with autologous blood, fluid replacement with colloid solution resulted in a lower Hct $(P<0.001)$, while crystalloid administration led to significant reductions in both Hct $(P=0.010)$ and $\mathrm{pH}$ $(P=0.009)$. No significant difference in the changes in $\mathrm{paO}_{2}$ and $\mathrm{pH}$ was observed between the rats in Groups CR and CO. The decreases in Hct were more pronounced in Group CO than those in Group CR $(P=0.032)$.

Figure 2 depicts the changes in the airway and respiratory tissue mechanics relative to the baseline. Blood withdrawal resulted in a systematic lowering of Raw. Fluid replacement with colloid in Group CO restored the baseline value of Raw, whereas the Raw remained reduced following the i.v. administration of autologous blood in Group B $(P=0.005)$. The changes in Raw after the i.v. infusion of crystalloid solution in Group CR were less marked $(P<0.038)$, with less obvious elevations in Raw after the third fluid replacement manoeuvre. Repetitive increases in $G$ were observed throughout the protocol $(P<0.001)$, with no statistically detectable differences between the protocol groups. $\mathrm{H}$ was elevated in all groups, with significantly greater changes in Groups CR $(P=0.005)$ and CO $(P=0.012)$ than in Group B.

The oedema indices obtained from the lung weights and from the histological analyses are seen in Fig. 3. The animals in both Groups CR and CO exhibited significantly greater wet-to-dry lung weight ratios $(P<0.001$ for both); this was also manifested in the perivascular pulmonary oedema indices $(P<0.05$ for both).

The systemic haemodynamic changes for the three groups of rats are displayed in Fig. 4. The blood withdrawals caused MAP to decrease systematically, and it was restored to previous values by the fluid replacements, regardless of the group allocation. HR gradually increased in all groups of rats, with significant changes from R3, W3 and R2 in Groups B, CR and CO, respectively.

The relationships between the wet-to-dry lung weight ratio and the relative change in $\mathrm{H}$ are presented in Fig. 5 . Pooling of the data from the three protocol groups revealed significant correlations between the macroscopic oedema index and the increased stiffness of the respiratory system $(r=0.55, P<0.01)$.

\section{Discussion}

Although recent studies have focused on the morbidity and mortality related to colloid or crystalloid use as fluid replacement therapy, ${ }^{1,2,14}$ the pulmonary effects of these solutions are mainly based on empirical investigations without firm evidence. ${ }^{5-7}$ Our experimental rat model was intended to mimic continuous insidious surgical bleeding and fluid replacement with solutions commonly used in clinical practice, and allowed us to assess mechanical properties specific for the airway and respiratory tissues following blood loss and replacement. The airway resistance decreased subsequent to the haemorrhage and remained low after fluid therapy with autologous blood, but returned to baseline following colloid and increased slightly after crystalloid. The respiratory tissues stiffened more markedly in the animals receiving colloid and crystalloid, with no difference in effect between these solutions. These adverse mechanical changes were also reflected in changes in the oedema indices determined by lung weight and by histology.

There has recently been an extensive debate concerning the best type and quantity of fluid replacement therapy following blood loss. Blood products are used to maintain the normal haemoglobin content of the circulation and ensure oxygen transport. Restoration of the circulatory blood volume by blood products has a beneficial effect on the preservation of the microcirculation with minimal morphological damage or ischaemic cell injury. ${ }^{15,16} \mathrm{In}$ common with previous findings, the blood loss in the present study led to bronchodilation, which is most probably due to the compensatory increase in thoracic gas volume and/or the elevated levels of circulatory catecholamines. ${ }^{8}$ Our findings add to what is known from a different model of haemorrhage that does not induce

Table 2 Mean (SD) values derived from arterial blood samples obtained at the first (W1), fourth (W4) and last (W6) withdrawal

\begin{tabular}{|c|c|c|c|c|c|c|c|c|c|}
\hline & \multicolumn{3}{|c|}{ Het $(\%)$} & \multicolumn{3}{|c|}{ pH } & \multicolumn{3}{|c|}{$\mathrm{paO}_{2}(\mathrm{mmHg})$} \\
\hline & $\mathrm{W}_{1}$ & W4 & W6 & W1 & W4 & W6 & $W_{1}$ & W4 & W6 \\
\hline Group B & $34.4(3.4)$ & $34.5(5.3)$ & $33.9(2.4)$ & $7.52(0.06)$ & $7.44(0.06)^{*}$ & $7.43(0.03)^{*}$ & $79.4(15.7)$ & $69.5(17.1)$ & $63.3(14.3)^{*}$ \\
\hline Group CO & 32.7 (3.6) & 31.7 (3.6) & $24.2(5.0)^{*, * *}$ & $7.51(0.06)$ & $7.40(0.03)^{*}$ & $7.40(0.06)^{*}$ & $80.4(8.7)$ & $54.9(7.0)^{*}$ & $63.7(6.7)^{*}$ \\
\hline Group CR & $33.8(2.3)$ & $31.1(7.2)$ & $28.5(5.3)^{*, * *, * * *}$ & $7.51(0.06)$ & $7.42(0.03)^{*}$ & $7.36(0.06)^{*, * *}$ & $79.9(16.8)$ & $63.0(7.8)^{*}$ & $64.9(12.3)^{*}$ \\
\hline
\end{tabular}

Hct, haematocrit; $\mathrm{paO}_{2}$, arterial partial pressure of oxygen. ${ }^{*} P<0.05$ vs. W1. ${ }^{* *} P<0.05$ vs. Group B. ${ }^{* * *} P<0.05$ vs. Group CO. 
Fig. 2
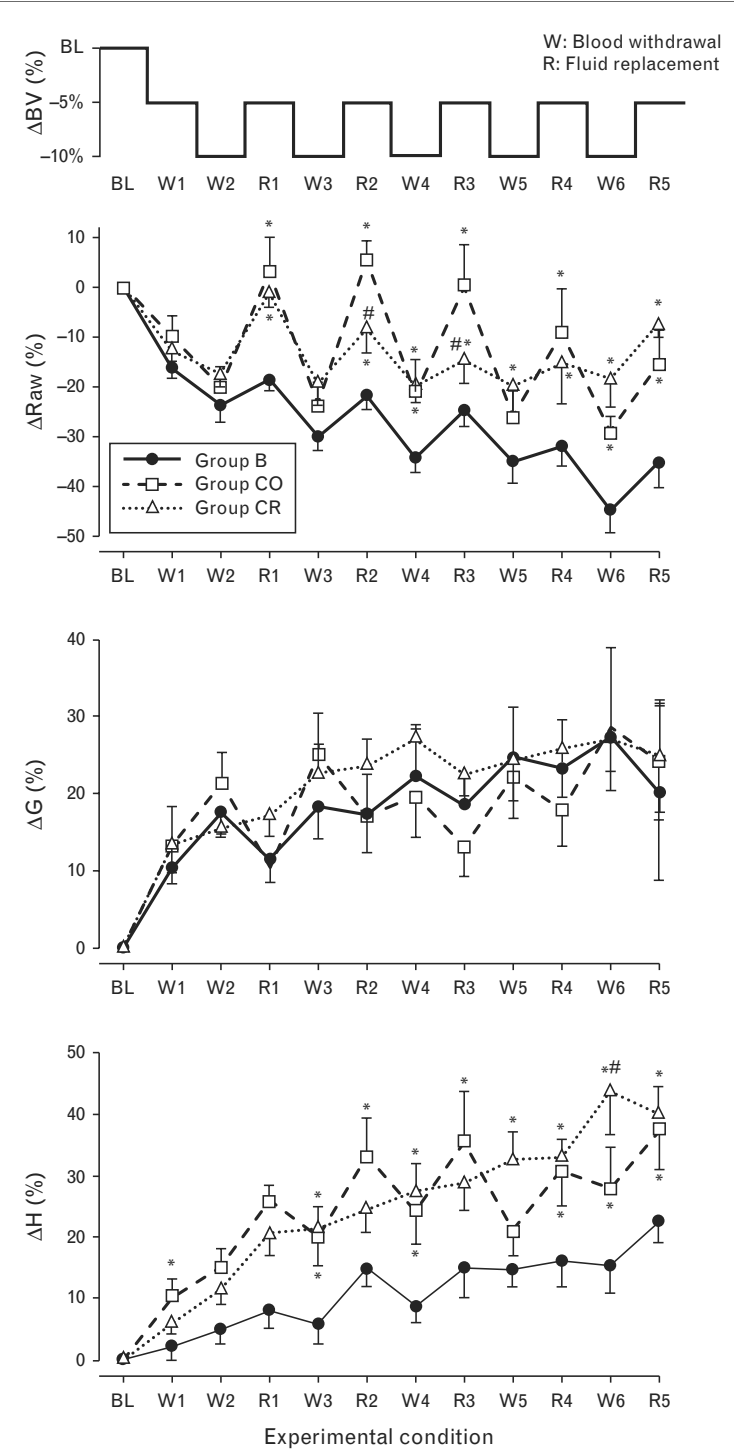

Changes in the airway (Raw: airway resistance) and tissue mechanics (G: damping, $\mathrm{H}$ : elastance) relative to the baseline (BL) during blood withdrawals (W1 to W6) and fluid replacements (R1 to R5) with

autologous blood (Group B), colloid (Group CO) or crystalloid (Group $\mathrm{CR})$. BV, total blood volume. ${ }^{*} P<0.05$ vs. Group B within a condition, ${ }^{\#} P<0.05$ vs. Group CO within a condition.

the severe hypovolaemia characteristic of hidden, leaky blood loss during major surgery.

Our results demonstrate that the Raw essentially remains lowered after administration of autologous blood. The lack of a complete recovery in airway tone could be attributed to the relaxation potential of heparin, ${ }^{17}$ but a comparison of heparinised and nonheparinised colloid solutions revealed no difference in their bronchial effects (data not shown), and the potential role of heparin can therefore be excluded. Alternatively, the depressed Raw may be attributed to the presence of bronchoactive mediators in the sequestered blood, with the particular importance of increased levels of adrenaline and noradrenaline in the withdrawn and subsequently re-administered blood. ${ }^{8}$ In contrast to autologous blood, colloid completely reversed the haemorrhage-induced bronchodilation. This illustrates the interaction between circulatory changes and airway mechanics, with recovery of the original airway geometry following restoration of normovolaemia. The increase in Raw following colloid administration may be attributed to a distension of the bronchial submucosal vessels and/or to oedema formation resulting in airway wall thickening, or an exudation into the airway lumen. ${ }^{18} \mathrm{~A}$ similar concept can be applied to the situation following crystalloid, the first administration of which fully reversed the decrease in Raw, when its entire volume was likely to remain in the vascular bed. This effect of the elevated intravascular volume may have been abolished in the rats of Group B due to the presence of catecholamines in the readministered autologous blood.

Following blood administration, there were slight, gradual increases in tissue viscoelasticity, which can be attributed to atelectasis and subsequent loss of lung volume induced by anaesthesia and mechanical ventilation in the supine position. This phenomenon was confirmed by the decrease in $\mathrm{paO}_{2}$, which indicates loss of alveolar surface available for gas exchange. An important finding of our study is the gradual, more marked impairment of respiratory tissue viscoelasticity in the animals receiving colloid or crystalloid solution (Fig. 2). This might be due to the different rheological properties of the fluids affecting the behaviour of respiratory tissue, ${ }^{19}$ or change in the colloid osmotic pressure related to haemodilution. As these adverse changes were also reflected in the oedema indices (Figs. 3 and 5), it is possible to anticipate that the accumulation of perivascular oedema will create stiffness in the compromised respiratory tissue. It is noteworthy that no difference was found between colloid and crystalloid treatments either in the changes in tissue mechanics or in the oedema indices. This suggests that, in terms of compromising lung tissue viscoelasticity and pulmonary oedema formation, these two solutions are equivalent, further supported by the lack of difference in the changes of blood oxygenation following the two fluid replacement regimens (Table 2). Studies that show little difference between colloid and crystalloid in effects on extravascular lung water, pulmonary leak index and lung injury score are in agreement. ${ }^{20-22}$ Our protocol covered only $90 \mathrm{~min}$, and although there might be an agreement within this period, the prolonged effects would require further study.

An important methodological aspect of our protocol is related to the nature and the volume of the fluid 
Fig. 3
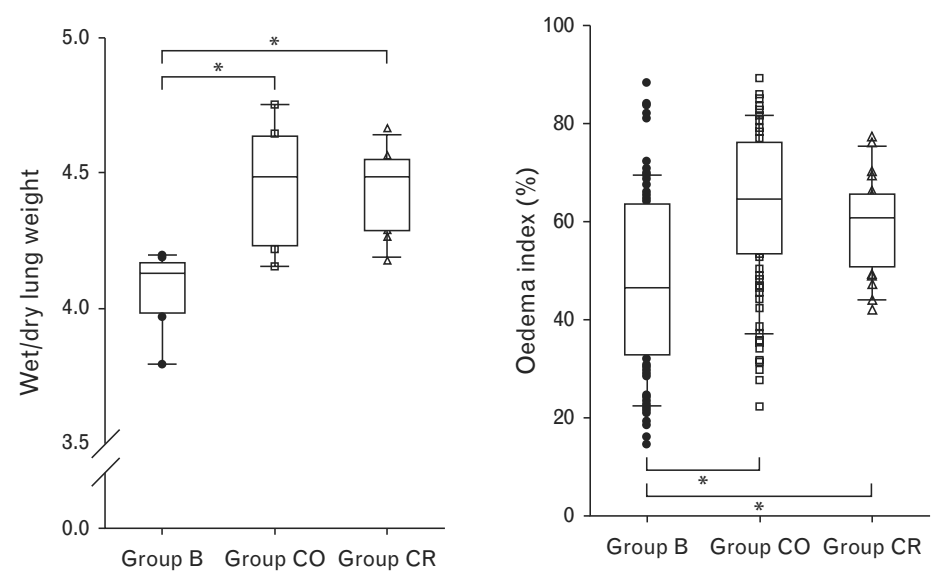

Oedema indices obtained by relating the wet lung weight to the dry weight (left) and by relating the perivascular oedema area to the total vessel area on histological sections obtained in rats receiving autologous blood (Group B), colloid (Group CO) or crystalloid (Group CR). $* P<0.05$.

Fig. 4
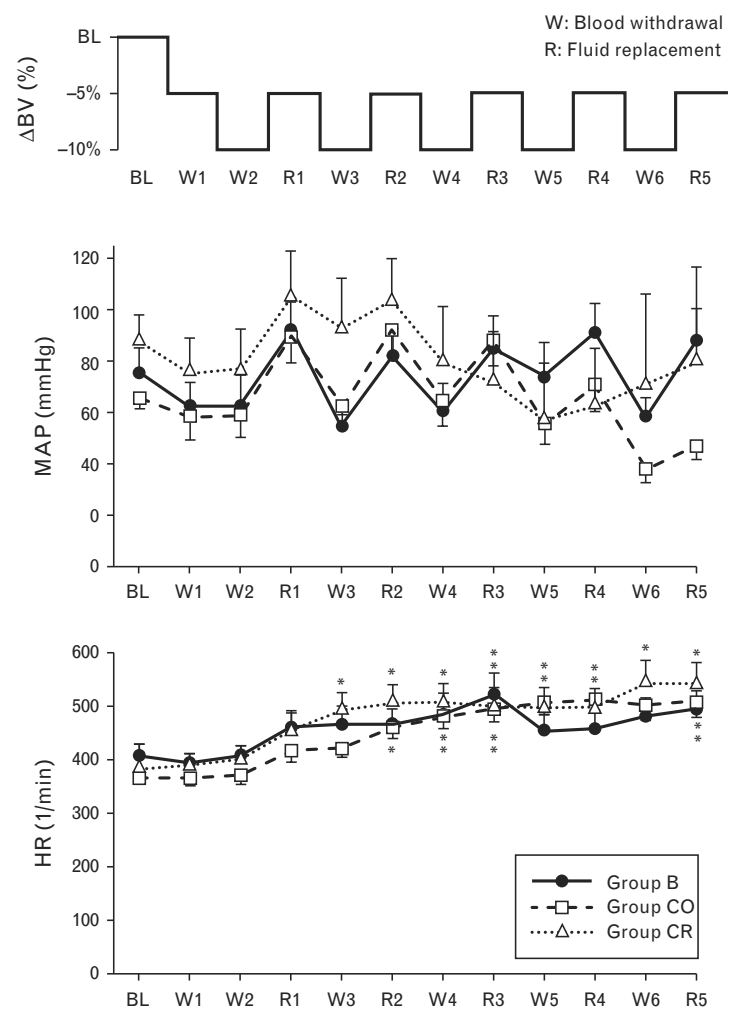

Systemic haemodynamic variables during blood withdrawals (W1 to W6) and fluid replacements (R1 to R5) with autologous blood (Group $\mathrm{B}$ ), colloid (Group CO) or crystalloid (Group CR). BV, total blood volume; HR, heart rate; MAP, mean arterial pressure; $\mathrm{BL}$, baseline. $* P<0.05$ vs. BL within a group. administered. There are a variety of fluids used in resuscitation, but no consensus as to which is the best. ${ }^{23}$ Some meta-analyses suggest that albumin is well tolerated in the critically ill, ${ }^{24}$ although others do not recommend it because of lack of robust evidence that it reduces mortality. ${ }^{25}$ A recent international consensus promotes crystalloids over both HES and albumin solutions. ${ }^{23}$ Because we wished to compare the effect of three basic fluid replacement strategies, we deliberately selected HES as the colloid solution comparator. For the crystalloid solution, we chose isotonic normal saline rather than the hypotonic Ringer's lactate. There is an evolving debate on the pros and cons of balanced salt solutions over normal saline; the latter matches our other two fluid

Fig. 5

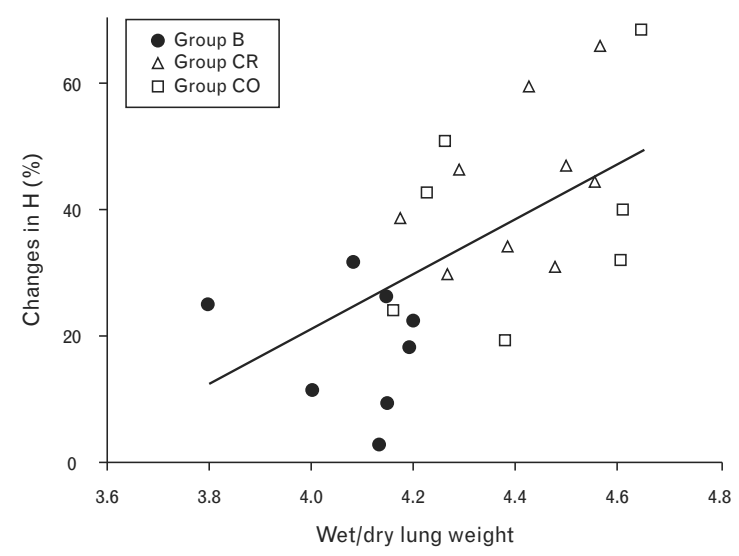

Relationship between the changes in oedema index (wet weight/dry weight) and in respiratory elastance $(\mathrm{H})$ in rats receiving autologous blood (Group B), colloid (Group CO) or crystalloid (Group CR).

Eur J Anaesthesio/ 2016; 33:34-41

Copyright $\odot 2015$ Wolters Kluwer Health, Inc. All rights reserved. 
replacement strategies for osmolarity and was selected for a comparison with the slightly hypertonic HES $6 \%$ $130 / 0.4{ }^{26}$ With regard to the volume of crystalloid solution for fluid replacement, no evidence-based recommendations are available. The conventional view is that the volume of crystalloid to be administered should be three to four times the blood loss, ${ }^{27}$ but recent studies have questioned this, suggesting a ratio close to $1: 1$. $^{2,7,28}$ As our interest was principally in the acute effects of fluid replacement, the volume administered for both solutions was the same as that chosen for the blood loss, $5 \%$ of the total blood volume. The similarity in MAP and HR between the protocol groups confirms this approach, and is in accord with the concept of goal-directed therapy. ${ }^{28,29}$

One methodological limitation of our study is the use of total respiratory impedance data to assess pulmonary changes. Although Raw accurately reflects the flow resistance of the airways, the chest wall contributes significantly to the tissue variables $\mathrm{G}$ and $\mathrm{H}^{12}$ Nevertheless, the viscoelastic properties of the chest wall exhibited negligible change following the induction of severe oedema with oleic acid. ${ }^{30}$ Therefore, we feel that our results probably reflect pulmonary changes; however, their magnitude may be somewhat underestimated due to the masking effect of the chest wall. Another methodological limitation is related to the species difference between small rodents and humans, necessitating caution in the extrapolation of our data to a clinical situation. Although rats have substantially higher Raw, $\mathrm{G}$ and $\mathrm{H}$ than humans, no major differences exist between mammalian species in the oscillatory mechanics apart from scaling factor. ${ }^{31}$

In summary, our results have provided experimental evidence of the dissociated changes in the airway and tissue mechanical properties following surgical-type bleeding and its treatment with autologous whole blood, colloid or crystalloid solution in a volume that fully restored MAP. Histological analysis and measurement of respiratory mechanics and gas exchange following blood loss and consecutive fluid replacement strategies revealed no differences between replacement with colloid and crystalloid. The two solutions demonstrated similar abilities to compromise the lung tissue viscoelasticity subsequent to mild perivascular oedema formation. These findings highlight the differences in behaviour of the respiratory system following fluid replacement with blood, colloid or crystalloid: a sustained bronchodilation is expected after the administration of autologous blood, without significant lung tissue changes, whereas colloids and crystalloids tend to restore the basal airway tone at the expense of deterioration in lung tissue viscoelasticity.

\section{Acknowledgments relating to this article}

Assistance with the study: the authors are indebted to Orsolya Ivánkovitsné Kiss for her invaluable assistance in the experiments.
The authors thank József Kaszaki for his excellent advice, and Gabriella Varga for her help in the surgical preparation.

Financial support and sponsorship: this work was supported by a Hungarian Scientific Research Grant (OTKA K81179). It was supported by the European Union and the State of Hungary, cofinanced by the European Social Fund in the framework of TÁMOP 4.2.4.A/2-11-1-2012-0001 'National Excellence Program' and 4.2.2.A-11/1/KONV-2012-0052.

Conflicts of interest: none.

Presentation: preliminary data were reported as an oral presentation at the annual congress of the European Respiratory Society in Munich, Germany, 6 to 10 September 2014.

\section{References}

1 Perel P, Roberts I, Ker K. Colloids versus crystalloids for fluid resuscitation in critically ill patients. Cochrane Database Syst Rev 2013; 2:CD000567.

Myburgh JA, Finfer S, Bellomo R, et al. Hydroxyethyl starch or saline for fluid resuscitation in intensive care. N Engl J Med 2012; 367:1901-1911.

3 Perner A, Haase N, Guttormsen AB, et al. Hydroxyethyl starch 130/0.42 versus Ringer's acetate in severe sepsis. NEngl J Med 2012; 367:124-134.

de Jonge $E$, Levi M. Effects of different plasma substitutes on blood coagulation: a comparative review. Crit Care Med 2001; 29:1261-1267.

5 Mcllroy DR, Kharasch ED. Acute intravascular volume expansion with rapidly administered crystalloid or colloid in the setting of moderate hypovolemia. Anesth Analg 2003; 96:1572-1577.

6 Hahn RG, Drobin D, Stahle L. Volume kinetics of Ringer's solution in female volunteers. Br J Anaesth 1997; 78:144-148.

7 Gondos T, Marjanek Z, Ulakcsai Z, et al. Short-term effectiveness of different volume replacement therapies in postoperative hypovolaemic patients. Eur J Anaesthesiol 2010; 27:794-800.

8 Bayat S, Albu G, Layachi S, et al. Acute hemorrhagic shock decreases airway resistance in anesthetized rat. J Appl Physio/ 2011; 111:458-464.

9 Petak F, Hantos Z, Adamicza A, et al. Methacholine-induced bronchoconstriction in rats: effects of intravenous vs. aerosol delivery. J Appl Physiol 1997; 82:1479-1487.

10 Franken H, Clement J, Cauberghs M, Van de Woestijne KP. Oscillating flow of a viscous compressible fluid through a rigid tube: a theoretical model. IEEE Trans Biomed Eng 1981; 28:416-420.

11 Hantos Z, Daroczy B, Suki B, et al. Input impedance and peripheral inhomogeneity of dog lungs. J Appl Physiol 1992; 72:168-178.

12 Petak F, Hall GL, Sly PD. Repeated measurements of airway and parenchymal mechanics in rats by using low-frequency oscillations. J Appl Physiol 1998; 84:1680-1686.

13 Lee HB, Blaufox MD. Blood volume in the rat. J Nucl Med 1985; 26:72-76.

14 Mutter TC, Ruth CA, Dart AB. Hydroxyethyl starch (HES) versus other fluid therapies: effects on kidney function. Cochrane Database Syst Rev 2013 7:CD007594.

15 Onen A, Cigdem MK, Deveci E, et al. Effects of whole blood, crystalloid, and colloid resuscitation of hemorrhagic shock on renal damage in rats: an ultrastructural study. J Pediatr Surg 2003; 38:1642-1649.

16 Appel PL, Shoemaker WC. Evaluation of fluid therapy in adult respiratory failure. Crit Care Med 1981; 9:862-869.

17 Abraham WM, Abraham MK, Ahmed T. Protective effect of heparin on immunologically induced tracheal smooth muscle contraction in vitro. Int Arch Allergy Immunol 1996; 110:79-84.

18 Widdicombe J. Physiologic control. Anatomy and physiology of the airway circulation. Am Rev Respir Dis 1992; 146:S3-S7.

19 Mogensen ML, Steimle KS, Karbing DS, Andreassen S. A model of perfusion of the healthy human lung. Comput Methods Programs Biomed $2011 ; 101: 156-165$

20 van der Heijden M, Verheij J, van Nieuw Amerongen GP, Groeneveld AB. Crystalloid or colloid fluid loading and pulmonary permeability, edema, and injury in septic and nonseptic critically ill patients with hypovolemia. Crit Care Med 2009; 37:1275-1281.

21 Pearl RG, Halperin BD, Mihm FG, Rosenthal MH. Pulmonary effects of crystalloid and colloid resuscitation from hemorrhagic shock in the presence of oleic acid-induced pulmonary capillary injury in the dog. Anesthesiology 1988; 68:12-20.

22 Hahn RG. Why are crystalloid and colloid fluid requirements similar during surgery and intensive care? Eur J Anaesthesio/ 2013; 30:515-518.

23 Raghunathan K, Murray PT, Beattie WS, et al. Choice of fluid in acute illness: what should be given? An international consensus. $\mathrm{Br} J$ Anaesth 2014; 113:772-783. 
24 Xu JY, Chen OH, Xie JF, et al. Comparison of the effects of albumin and crystalloid on mortality in adult patients with severe sepsis and septic shock: a meta-analysis of randomized clinical trials. Crit Care 2014; 18:702.

25 Patel A, Laffan MA, Waheed U, Brett SJ. Randomised trials of human albumin for adults with sepsis: systematic review and meta-analysis with trial sequential analysis of all-cause mortality. BMJ 2014; 349:g4561.

26 Ertmer $\mathrm{C}$, Van Aken $\mathrm{H}$. Fluid therapy in patients with brain injury: what does physiology tell us? Crit Care 2014; 18:119.

27 Puyana JC. Resuscitation of hypovolemic shock. In: Fink MP, editor. Textbook of critical care. Philadelphia, PA: Elsevier Saunders; 2005. pp. $1939-1940$
28 Hiltebrand LB, Kimberger O, Arnberger M, et al. Crystalloids versus colloids for goal-directed fluid therapy in major surgery. Crit Care 2009; 13:R40

29 Doherty M, Buggy DJ. Intraoperative fluids: how much is too much? $\mathrm{Br} J$ Anaesth 2012; 109:69-79.

30 Barnas GM, Stamenovic D, Lutchen KR. Lung and chest wall impedances in the dog in normal range of breathing: effects of pulmonary edema. J App/ Physiol 1992; 73:1040-1046.

31 Bates JH, Irvin CG, Farre R, Hantos Z. Oscillation mechanics of the respiratory system. Compr Physiol 2011; 1:1233-1272. 